\title{
Nonlinear resonance interaction between conjugate circumferential flexural modes in single-walled carbon nanotubes
}

\author{
Matteo Strozzi* ${ }^{*}$ Francesco Pellicano ${ }^{\circ}$ \\ *Department of Sciences and Methods for Engineering, University of Modena and Reggio Emilia, \\ Via Giovanni Amendola 2, 42122 Reggio Emilia, Italy \\ 'Department of Engineering “Enzo Ferrari”, University of Modena and Reggio Emilia, \\ Via Pietro Vivarelli 10/1, 41125 Modena, Italy
}

N. of Pages: 54

N. of Figures: 25

N. of Tables: 6

Corresponding Author

Dr. Eng. Matteo Strozzi, Ph.D.

Department of Sciences and Methods for Engineering,

University of Modena and Reggio Emilia,

Via Giovanni Amendola 2, 42122 Reggio Emilia, Italy

Email: matteo.strozzi@unimore.it 


\begin{abstract}
This paper presents an investigation on the dynamical properties of single-walled carbon nanotubes (SWCNTs), nonlinear modal interaction and energy exchange are analysed in detail. Resonance interactions between two conjugate circumferential flexural modes (CFMs) are investigated. The nanotubes are analysed through a continuous shell model, a thin shell theory is used to model the dynamics of the system; free-free boundary conditions are considered. The Rayleigh-Ritz method is applied to approximate linear eigenfunctions of the partial differential equations that govern the shell dynamics. An energy approach, based on Lagrange equations and series expansion of the displacements, is considered to reduce the initial partial differential equations to a set of nonlinear ordinary differential equations of motion. The model is validated in linear field (natural frequencies) by means of comparisons with literature. A convergence analysis is carried out in order to obtain the smallest modal expansion able to simulate the nonlinear regimes. The time evolution of the nonlinear energy distribution over the SWCNT surface is studied. The nonlinear dynamics of the system is analysed by means of phase portraits. The resonance interaction and energy transfer between the conjugate CFMs is investigated. A travelling wave moving along the circumferential direction of the SWCNT is observed.
\end{abstract}

\title{
Keywords
}

Nonlinear oscillations, resonance interaction, conjugate modes, carbon nanotubes 


\section{Introduction}

Carbon nanotubes (CNTs) were first discovered in 1991 by S. Iijima in the laboratories of the NEC Corporation in Japan. These "helical microtubules of graphitic carbon" were observed in the form of needles comprising several coaxial tubes of graphitic sheets by means of a transmission electron microscopy (TEM). They were grown on the negative end of the carbon electrode used in the arcdischarge synthesis of fullerenes and were detected by high-resolution electron micrographs [1].

Starting from their discovery, CNTs have attracted increasing interest from researchers all over the world because of their outstanding mechanical properties, in particular very high Young's modulus (of the order of 1-2 TPa) and tensile strength (up to $100 \mathrm{GPa}$ ) [2-4].

The combination of the two previous mechanical properties allows CNTs to reach natural vibration frequencies of the order of THz: these ultra-high frequencies have led to experiment the application of CNTs and their composites as ultra-high sensitivity resonators within nano-electro-mechanicalsystems (NEMS), such as oscillators, sensors and charge detectors [5-10].

Several methodologies have been adopted in order to better investigate the mechanical properties of CNTs and their application in many industrial fields. Experimental tests, numerical simulations and continuum mechanics studies have been performed. Single-walled and multi-walled (MWCNTs) carbon nanotubes have been considered.

Experiments based on the resonant Raman spectroscopy (RRS) technology have been carried out to obtain atomic structure and chirality of SWCNTs considering the frequency spectrum of the CNT and applying the theory of the resonant transitions. This method starts from the measurement of the SWCNT diameter obtained by means of atomic force microscopy (AFM) and investigates natural frequency and energy of the SWCNT radial breathing mode (RBM) [11-15].

However, owing to their high technological complexity and costs, experimental methods cannot be considered as efficient approaches to investigate the mechanical behaviour of CNTs.

Molecular dynamics simulations (MDS) have been performed to get numerically the time evolution of SWCNT as a system of atoms treated as point-like masses interacting with one another according to an assumed potential energy which describes the atomic bond interactions along the CNT. The vibrations of the free atoms of the SWCNT are recorded for a certain duration at fixed temperature and the corresponding natural frequencies are computed by the Discrete Fourier Transform (DFT) [16-20]. Modelling the SWCNT as an equivalent space frame-like discrete structure, on the basis of atomistic structural mechanics (MDS), is computationally very expensive and this method cannot be easily applied to the structural simulation of CNTs, which incorporates a large number of atoms. Theoretical models based on continuum mechanics are computationally more efficient than MDS. In such kind of modelling, CNTs are treated as continuous homogeneous structures, ignoring their 
intrinsic atomic nature, this allows the achievement of a strong reduction of the number of degrees of freedom. In order to investigate the vibration properties of SWCNTs and MWCNTs, both beamlike [21-33] and shell-like [34-38] equivalent continuous models have been proposed in the past.

In particular, the analogy between circular cylindrical shell and CNT nanostructures led to extensive application of continuous shell models for the CNT vibration analysis. To deepen the knowledge on shells, the fundamental books of Leissa [39] and Yamaki [40] are suggested to the reader. In these works, nonlinear dynamics and stability of shells with different shapes, material properties and boundary conditions are treated, the most important thin shell theories (Donnell, Sanders-Koiter, Flugge-Lur'e-Byrne) are reported, numerical and experimental results are described.

In order to study the CNT dynamics through continuous models, equivalent mechanical parameters (i.e., Young's modulus, Poisson's ratio and mass density) must be applied. Indeed, we are going to simulate the intrinsically discrete CNT with its continuous twin: a circular cylindrical shell; in order to be dynamically equivalent all shell parameters must be suitably set, this was achieved comparing the equations of the classical shell theory for tensile and bending rigidity with the MDS results [41]. One of the most controversial topics in modelling the discrete CNTs as continuous cylindrical shells is denoted by the inclusion of the size effects, i.e. surface stresses, strain gradients and nonlocalities, into the elastic shell theory considered. It was found in literature [42] that the size effects influence the vibration characteristics of the CNTs in the higher region of their frequency spectrum (vibration modes with relatively high number of longitudinal half-waves). Therefore, to obtain relatively high resonant frequencies correctly, nonlocal elastic shell models were developed, where the size effects are taken into account in the stress-strain relationships [43-45].

Both theoretical and experimental aspects of nonlinear dynamics and stability of shells are widely explored in literature [46-50]. In these works, the effect of the geometry, boundary conditions, fluid-structure interaction, material distribution, geometric imperfections, external loads on the nonlinear vibrations of circular cylindrical shells is investigated. Particular attention was paid to the choice of modes used in the expansion of the displacements, the general goal is to simulate the nonlinear dynamic behaviour of the shell with a minimum number of degrees of freedom: these models were obtained by means of convergence analyses of the nonlinear response of the system, see Refs. [51-53] for more details.

It must be stressed that the application of the CNTs and their composites as ultra-high sensitivity resonators within NEMS requires deep investigation of the nonstationary dynamics phenomena, i.e., energy transfers, internal resonances and propagating waves, in the CNTs, considered as strongly nonlinear systems. To this aim, the nonlinear resonance interactions and energy exchanges between vibration modes in SWCNTs were extensively studied in the literature [54-58]. 
An interesting nonstationary dynamic phenomenon, a travelling wave response, was observed both numerically and experimentally in circular cylindrical shells, with and without fluid-structure interaction [59-60]. This phenomenon is due to the coexistence of three specific conditions: i) axisymmetric structure, ii) nonlinear vibrations, iii) conjugate modes. Shells with circular cylindrical geometry are symmetric with respect to the longitudinal axis; in presence of large amplitude displacements, they are subjected to nonlinear vibrations; conjugate modes are couples of modes having the same mode shape and frequency, but angularly shifted of a quarter of period in circumferential direction. Therefore, in circular cylindrical shell, the nonlinear resonance interaction between conjugate modes can give rise to a pure travelling wave moving along the circumferential direction of the shell. To the authors' best knowledge, the present paper is the first work in which the travelling wave response in SWCNT, considered as "graphene sheet rolled into a seamless tube" [1], is observed in nonlinear field.

In this paper, the nonlinear oscillations and energy distribution of SWCNTs are investigated. The resonance interaction between two CFMs having the same natural frequency and with mode shapes shifted of $\pi / 4$ (conjugate modes) is analysed. The Sanders-Koiter shell theory is applied to model the nonlinear dynamics of the system in case of finite amplitude vibration. The CNT deformation is given in terms of longitudinal, circumferential and radial displacement field. Free-free boundary conditions are considered. In the linear analysis, the three displacements are expanded by using a double mixed series and the Rayleigh-Ritz method is applied to get approximated eigenfunctions. In the nonlinear analysis, the three displacements are re-expanded by considering the approximated eigenfunctions of the linear analysis and an energy method based on the Lagrange equations is used to reduce the partial differential equations of motion to a system of ordinary differential equations, which is then solved by means of the implicit Runge-Kutta numerical scheme. The model is validated by comparing the natural frequencies with the results of experiments and molecular dynamics simulations found in the literature. A convergence analysis is carried out to select the vibration modes to be added to the two directly excited CFMs providing a proper description of the nonlinear response of the system. The evolution in time of the nonlinear energy distribution of the conjugate CFMs over the SWCNT surface is studied. Phase portraits describing the nonlinear dynamics of the system are found. The effect of the conjugate mode resonance interaction on the nonlinear behaviour of the SWCNT is investigated. The participation of both conjugate modes gives a pure travelling wave moving circumferentially around the CNT. 


\section{Sanders-Koiter shell theory}

In this work, the Sanders-Koiter elastic thin shell theory is used to model the SWCNT dynamics.

It must be stressed that, in the present paper, the contribution of the size effects (surface stresses, strain gradients and nonlocalities) is not taken into account in the stress-strain relationships.

Since size effects have a relevant influence in the higher region of the SWCNT frequency spectrum (vibration modes with relatively high number of longitudinal half-waves and resonant frequencies), then they can be neglected in this manuscript, which is focused on the lower region of the SWCNT frequency spectrum (conjugate CFMs with relatively low number of longitudinal half-waves and resonant frequencies). Therefore, in the present work, the local continuum mechanics neglecting the size effects can be applied correctly without loss of accuracy, see Ref. [42] for more details.

In Figure 1(a), a picture of a discrete SWCNT is added to better describe the transition from the actual atomistic structure of the CNT to the equivalent continuous shell model adopted in this work. In Figure 1(b,c), a circular cylindrical shell having radius $R$, length $L$ and thickness $h$ is represented; a cylindrical coordinate system $(O ; x, \theta, z)$ is considered where the origin $O$ of the reference system is located at the centre of one end of the shell. In Figure 1(b,c), three displacements are represented: longitudinal $u(x, \theta, t)$, circumferential $v(x, \theta, t)$ and radial $w(x, \theta, t)$; the radial displacement $w$ is considered positive outward; $(x, \theta)$ are the longitudinal and angular coordinates of an arbitrary point on the middle surface of the shell; $z$ is the radial coordinate along the thickness $h ; t$ is the time.

(a)

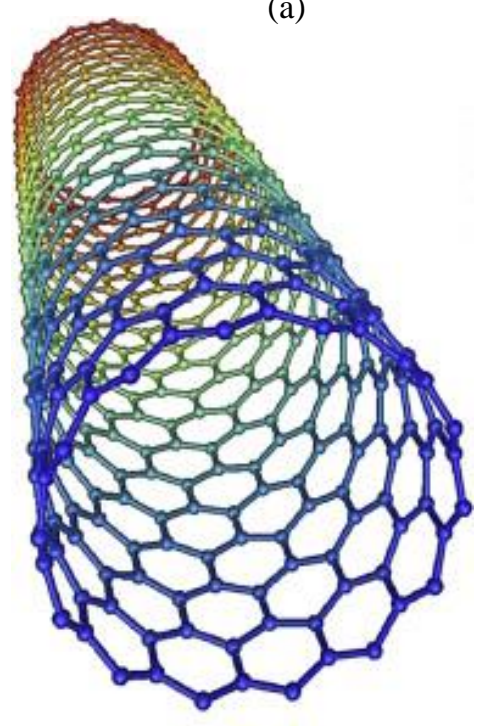

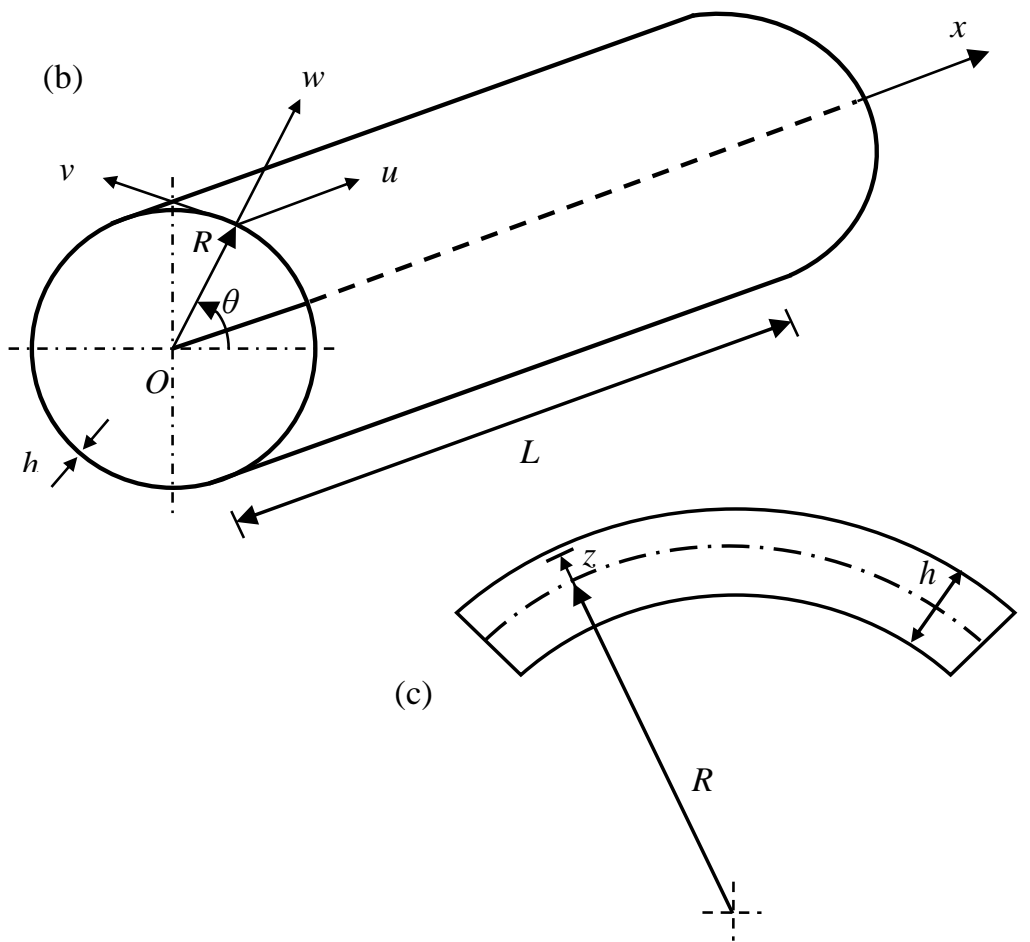

Figure 1. Equivalent continuum structure of a SWCNT. (a) Discrete SWCNT [19]. (b) Geometry of the equivalent circular cylindrical shell. (c) Cross-section of the circular cylindrical shell surface. 


\subsection{Strain-displacement relationships}

Since one of the most important issues of CNT continuum modelling is given by the infinitesimal dimensions of the structure, which cause very fast time scales, inducing therefore hard numerical troubles into the numerical integration method, then, in this work, the governing parameters, i.e., shell displacements and time, are transformed into dimensionless form.

The dimensionless displacement field $(\tilde{u}, \tilde{v}, \tilde{w})$ of the shell can be written in the form [38]

$$
\tilde{u}=\frac{u}{R} \quad \tilde{v}=\frac{v}{R} \quad \tilde{w}=\frac{w}{R}
$$

where $(u, v, w)$ is the dimensional displacement field and $R$ is the radius of the shell.

The Sanders-Koiter shell theory is based on the Love's first approximation, which considers the following five hypotheses [39]: (i) the thickness of the shell is very small in comparison with the other dimensions, (ii) the deformations are small, (iii) the transverse normal stress is small with respect to the other stresses and can be neglected $\left(\sigma_{z}=0\right)$, (iv) the normal to the reference undeformed shell surface remains straight and normal to the deformed surface, and (v) the normal to the reference undeformed surface suffers no extension during deformation, i.e., no thickness stretching is present (Kirchhoff-Love kinematic hypothesis).

The consequences of the last two assumptions are that the transverse shear deformations of the shell are neglected $\left(\gamma_{x z}=\gamma_{\theta z}=0\right)$; in addition, also the rotary inertia of the shell is neglected.

The dimensionless middle surface strains of the shell $\left(\tilde{\varepsilon}_{x, 0}, \tilde{\varepsilon}_{\theta, 0}, \tilde{\gamma}_{x \theta, 0}\right)$ are expressed as [55]

$$
\begin{gathered}
\tilde{\varepsilon}_{x, 0}=\left[\alpha \frac{\partial \tilde{u}}{\partial \eta}\right]+\left\{\frac{1}{2} \alpha^{2}\left(\frac{\partial \tilde{w}}{\partial \eta}\right)^{2}+\frac{1}{8}\left(\alpha \frac{\partial \tilde{v}}{\partial \eta}-\frac{\partial \tilde{u}}{\partial \theta}\right)^{2}\right\} \\
\tilde{\varepsilon}_{\theta, 0}=\left[\frac{\partial \tilde{v}}{\partial \theta}+\tilde{w}\right]+\left\{\frac{1}{2}\left(\frac{\partial \tilde{w}}{\partial \theta}-\tilde{v}\right)^{2}+\frac{1}{8}\left(\frac{\partial \tilde{u}}{\partial \theta}-\alpha \frac{\partial \tilde{v}}{\partial \eta}\right)^{2}\right\} \\
\tilde{\gamma}_{x \theta, 0}=\left[\frac{\partial \tilde{u}}{\partial \theta}+\alpha \frac{\partial \tilde{v}}{\partial \eta}\right]+\left\{\alpha \frac{\partial \tilde{w}}{\partial \eta}\left(\frac{\partial \tilde{w}}{\partial \theta}-\tilde{v}\right)\right\}
\end{gathered}
$$

where the linear terms are inserted into square brackets, the nonlinear terms are inserted into curly brackets, $\eta=x / L$ is the dimensionless longitudinal coordinate of the shell and $\alpha=R / L$. 
The separation of the linear and nonlinear terms in equations (2-4) can be useful in order to properly insert the strain contributions into the equation of the dimensionless elastic strain energy of the shell in the following linear and nonlinear vibration analyses.

The dimensionless middle surface changes in curvature and torsion of the shell $\left(\tilde{k}_{x}, \tilde{k}_{\theta}, \tilde{k}_{x \theta}\right)$ are linear terms given by [55]

$$
\tilde{k}_{x}=-\alpha^{2} \frac{\partial^{2} \tilde{w}}{\partial \eta^{2}} \quad \tilde{k}_{\theta}=\frac{\partial \tilde{v}}{\partial \theta}-\frac{\partial^{2} \tilde{w}}{\partial \theta^{2}} \quad \tilde{k}_{x \theta}=-2 \alpha \frac{\partial^{2} \tilde{w}}{\partial \eta \partial \theta}+\frac{3}{2} \alpha \frac{\partial \tilde{v}}{\partial \eta}-\frac{1}{2} \frac{\partial \tilde{u}}{\partial \theta}
$$

\subsection{Force and moment resultants}

The dimensionless force $\left(\tilde{N}_{x}, \tilde{N}_{\theta}, \tilde{N}_{x \theta}, \tilde{Q}_{x}, \tilde{Q}_{\theta}\right)$ and moment $\left(\tilde{M}_{x}, \tilde{M}_{\theta}, \tilde{M}_{x \theta}\right)$ resultants per unit length of the shell are expressed as [38]

$$
\begin{array}{ccc}
\tilde{N}_{x}=\frac{1}{\left(1-v^{2}\right)}\left(\tilde{\varepsilon}_{x, 0}+v \tilde{\varepsilon}_{\theta, 0}\right) & \tilde{N}_{\theta}=\frac{1}{\left(1-v^{2}\right)}\left(\tilde{\varepsilon}_{\theta, 0}+v \tilde{\varepsilon}_{x, 0}\right) & \tilde{N}_{x \theta}=\frac{1}{2(1+v)} \tilde{\gamma}_{x \theta, 0} \\
\tilde{M}_{x}=\frac{\beta}{12\left(1-v^{2}\right)}\left(\tilde{k}_{x}+v \tilde{k}_{\theta}\right) & \tilde{M}_{\theta}=\frac{\beta}{12\left(1-v^{2}\right)}\left(\tilde{k}_{\theta}+v \tilde{k}_{x}\right) & \tilde{M}_{x \theta}=\frac{\beta}{24(1+v)} \tilde{k}_{x \theta} \\
\tilde{Q}_{x}=\frac{\beta^{2}}{12\left(1-v^{2}\right)}\left(\alpha\left(\tilde{k}_{x, x}+v \tilde{k}_{\theta, x}\right)+\frac{(1-v)}{2} \tilde{k}_{x \theta, \theta}\right) & \tilde{Q}_{\theta}=\frac{\beta^{2}}{12\left(1-v^{2}\right)}\left(\frac{(1-v)}{2} \alpha \tilde{k}_{x \theta, x}+\tilde{k}_{\theta, \theta}+v \tilde{k}_{x, \theta}\right)
\end{array}
$$

where $v$ is the Poisson's ratio of the shell and $\beta=h / R$. These expressions of the dimensionless force and moment resultants will be used into the equations of the natural-type boundary conditions.

\subsection{Elastic strain energy}

In the case of homogeneous and isotropic material, the dimensionless elastic strain energy $\tilde{U}$ of a cylindrical shell, by neglecting the transverse normal stress $\sigma_{z}$ (Love's first approximation), can be written as a function of the dimensionless strains $\left(\tilde{\varepsilon}_{x, 0}, \tilde{\varepsilon}_{\theta, 0}, \tilde{\gamma}_{x \theta, 0}\right)$ and $\left(\tilde{k}_{x}, \tilde{k}_{\theta}, \tilde{k}_{x \theta}\right)$ in the form [38]

$$
\begin{aligned}
\tilde{U}(\eta, \theta, \tau)= & \frac{1}{2} \frac{1}{\left(1-v^{2}\right)}\left(\int_{0}^{1} \int_{0}^{2 \pi}\left(\tilde{\varepsilon}_{x, 0}^{2}+\tilde{\varepsilon}_{\theta, 0}^{2}+2 v \tilde{\varepsilon}_{x, 0} \tilde{\varepsilon}_{\theta, 0}+\frac{(1-v)}{2} \tilde{\gamma}_{x \theta, 0}^{2}\right) d \eta d \theta+\right. \\
& \left.+\frac{\beta^{2}}{12} \int_{0}^{1} \int_{0}^{2 \pi}\left(\tilde{k}_{x}^{2}+\tilde{k}_{\theta}^{2}+2 v \tilde{k}_{x} \tilde{k}_{\theta}+\frac{(1-v)}{2} \tilde{k}_{x \theta}^{2}\right) d \eta d \theta\right)
\end{aligned}
$$


where the first term of the right-hand side of equation (9) is the membrane energy (also referred to stretching energy) and the second one is the bending energy.

Another relevant issue of the thin shell modelling of SWCNTs is given by the choice of an isotropic or anisotropic model; indeed, CNTs are discrete systems, i.e., they are intrinsically non-isotropic. However, it was proven in the past that the use of isotropic models for SWCNTs does not lead to significant errors. Therefore, in the present work, an isotropic elastic shell model is considered.

\subsection{Kinetic energy}

The dimensionless time variable $\tau$ is defined introducing a reference dimensional circular frequency $\omega_{0}$ (i.e., the lowest extensional circular frequency of a ring in plane strain) in the form [57]

$$
\tau=\omega_{0} t
$$

$$
\omega_{0}=\sqrt{\frac{E}{\left(1-v^{2}\right) \rho R^{2}}}
$$

where $t$ is the dimensional time, $(E, \rho)$ are the Young's modulus and mass density of the shell. The dimensionless velocity field $(\tilde{u}, \tilde{v}, \tilde{w})$ of the shell is given by [57]

$$
\tilde{u}^{\prime}=\frac{d \tilde{u}}{d \tau}=\frac{\dot{u}}{R \omega_{0}} \quad \tilde{v}^{\prime}=\frac{d \tilde{v}}{d \tau}=\frac{\dot{v}}{R \omega_{0}} \quad \tilde{w}^{\prime}=\frac{d \tilde{w}}{d \tau}=\frac{\dot{w}}{R \omega_{0}}
$$

where $(\dot{u}, \dot{v}, \dot{w})$ is the dimensional velocity field.

The dimensionless kinetic energy $\tilde{T}$ of a cylindrical shell (rotary inertia is neglected) is [57]

$$
\tilde{T}(\eta, \theta, \tau)=\frac{1}{2} \gamma \int_{0}^{1} \int_{0}^{2 \pi}\left(\tilde{u}^{2}+\tilde{v}^{12}+\tilde{w}^{\prime 2}\right) d \eta d \theta
$$

where $\gamma=\rho R^{2} \omega_{0}^{2} / E$.

\subsection{Total energy}

The dimensionless total energy $\tilde{E}$ of the shell can be expressed as the sum of the dimensionless elastic strain energy $\tilde{U}$ and kinetic energy $\tilde{T}$ in the following form

$$
\tilde{E}(\eta, \theta, \tau)=\tilde{U}(\eta, \theta, \tau)+\tilde{T}(\eta, \theta, \tau)
$$




\section{Vibration analysis}

In order to study the linear and nonlinear vibrations of the shell, a two-step energy based procedure is adopted: i) in the linear analysis, the three displacements are expanded by using a double mixed series, the potential and kinetic energies are developed in terms of the series free parameters and the Rayleigh-Ritz method is considered to obtain approximated eigenfunctions; ii) in the nonlinear analysis, the three displacements are re-expanded by using the approximated eigenfunctions of the linear analysis, the potential and kinetic energies are developed in terms of modal coordinates and the Lagrange equations are applied to obtain a system of nonlinear ordinary differential equations of motion, which is solved numerically.

\subsection{Linear vibration analysis}

The linear vibration analysis is carried out by considering only the quadratic terms into the equation of the dimensionless elastic strain energy (9) and adopting the same procedure of Refs. $[38,46]$. A modal vibration, i.e., a synchronous motion, can be formally written in the form [38]

$$
\tilde{u}(\eta, \theta, \tau)=\tilde{U}(\eta, \theta) \tilde{f}(\tau) \quad \tilde{v}(\eta, \theta, \tau)=\tilde{V}(\eta, \theta) \tilde{f}(\tau) \quad \tilde{w}(\eta, \theta, \tau)=\tilde{W}(\eta, \theta) \tilde{f}(\tau)
$$

where $\tilde{u}(\eta, \theta, \tau), \tilde{v}(\eta, \theta, \tau), \tilde{w}(\eta, \theta, \tau)$ are the three dimensionless displacements of the cylindrical shell, $\tilde{U}(\eta, \theta), \tilde{V}(\eta, \theta), \tilde{W}(\eta, \theta)$ describe the corresponding dimensionless mode shape and $\tilde{f}(\tau)$ is the dimensionless time law, which is supposed to be the same for each displacement in the modal vibration analysis (synchronous motion hypothesis).

The dimensionless mode shape $(\tilde{U}, \tilde{V}, \tilde{W})$ is expanded by considering a double mixed series in terms of $m$-th order dimensionless Chebyshev polynomials $\tilde{T}_{m}^{*}(\eta)$ along the longitudinal direction and harmonic functions $(\cos n \theta, \sin n \theta)$ along the circumferential direction; following Ref. [38], the expansion reads:

$$
\begin{gathered}
\tilde{U}(\eta, \theta)=\sum_{m=0}^{M_{u}} \sum_{n=0}^{N} \tilde{U}_{m, n} \tilde{T}_{m}^{*}(\eta) \cos n \theta \\
\tilde{V}(\eta, \theta)=\sum_{m=0}^{M_{v}} \sum_{n=0}^{N} \tilde{V}_{m, n} \tilde{T}_{m}^{*}(\eta) \sin n \theta \\
\tilde{W}(\eta, \theta)=\sum_{m=0}^{M_{w}} \sum_{n=0}^{N} \tilde{W}_{m, n} \tilde{T}_{m}^{*}(\eta) \cos n \theta
\end{gathered}
$$


where $\tilde{T}_{m}^{*}(\eta)=\tilde{T}_{m}(2 \eta-1), m$ denotes the degree of the Chebyshev polynomial and $n$ is the number of nodal diameters.

It must be noted that $\left(\tilde{U}_{m, n}, \tilde{V}_{m, n}, \tilde{W}_{m, n}\right)$ are unknown dimensionless coefficients which are found by imposing the boundary conditions, i.e., by applying constraints to the expansions (15-17).

Due to the axial symmetry and the isotropy of the system, in the absence of imperfections and in the case of axisymmetric boundary conditions (e.g., simply, clamped, free), the harmonic functions are orthogonal with respect to the linear operator of the shell in the circumferential direction $\theta$ and the mode shapes are characterized by a specific number of nodal diameters $n$; therefore, the double series of equations (15-17) can be reduced to a single series and the linear mode shape can assume the following simplified expression [51]

$$
\begin{array}{rc}
\tilde{U}(\eta, \theta)=\sum_{m=0}^{M_{u}} \tilde{U}_{m, n} \tilde{T}_{m}^{*}(\eta) \cos n \theta & V(\eta, \theta)=\sum_{m=0}^{M_{v}} \tilde{V}_{m, n} T_{m}^{*}(\eta) \sin n \theta \\
W(\eta, \theta)=\sum_{m=0}^{M_{w}} \tilde{W}_{m, n} T_{m}^{*}(\eta) \cos n \theta & n=0,1,2, \ldots, N
\end{array}
$$

The dimensionless mode shape $\left(\tilde{U}_{c}, \tilde{V}_{c}, \tilde{W}_{c}\right)$ of the corresponding conjugate mode is given by

$$
\begin{gathered}
\tilde{U}_{c}(\eta, \theta)=\sum_{m=0}^{M_{u}} \sum_{n=1}^{N} \tilde{U}_{m, n} \tilde{T}_{m}^{*}(\eta) \sin n \theta \\
\tilde{V}_{c}(\eta, \theta)=-\sum_{m=0}^{M_{v}} \sum_{n=1}^{N} \tilde{V}_{m, n} \tilde{T}_{m}^{*}(\eta) \cos \operatorname{s} n \theta \\
\tilde{W}_{c}(\eta, \theta)=\sum_{m=0}^{M_{w}} \sum_{n=1}^{N} \tilde{W}_{m, n} \tilde{T}_{m}^{*}(\eta) \sin n \theta
\end{gathered}
$$

From expansions (19-21), it can be noted that the mode shape of the conjugate mode is rotated by an angle equal to $\pi /(2 n)$ with respect to the mode shape described in expansions (15-17). Moreover, expansions (19-21) do not include the axisymmetric modes $(n=0)$, which are taken into account in the previous ones (15-17).

\subsection{Boundary conditions}

Free-free boundary conditions are given by the following equations [38] 


$$
\tilde{N}_{x}=0 \quad \tilde{N}_{x \theta}+\frac{\tilde{M}_{x \theta}}{R}=0 \quad \tilde{Q}_{x}+\frac{1}{R} \frac{\partial \tilde{M}_{x \theta}}{\partial \theta}=0 \quad \tilde{M}_{x}=0 \quad \text { for } \eta=0,1
$$

where the expressions of the dimensionless force $\left(\tilde{N}_{x}, \tilde{N}_{x \theta}, \tilde{Q}_{x}\right)$ and moment $\left(\tilde{M}_{x}, \tilde{M}_{x \theta}\right)$ resultants per unit length are given in equations (6-8).

It can be observed from equations (22) that the free-free boundary conditions are of natural type, i.e. they involve forces and moments only. Therefore, it is not strictly necessary that the expansions (15-17) respect such constraints; indeed, it is well known that the Rayleigh-Ritz method can be properly applied to obtain approximated eigenfunctions even if the natural boundary conditions are not respected.

In the present paper, focused on free-free SWCNTs, no boundary equations are imposed and no constraints are applied to the unknown coefficients of expansions (15-17).

\subsection{Rayleigh-Ritz method}

The maximum number of variables needed for describing a vibration mode with $n$ nodal diameters is $N_{p}=M_{u}+M_{v}+M_{w}+3-p$, where $M_{u}=M_{v}=M_{w}$ denote the degree of the Chebyshev polynomials and $p$ describes the number of equations needed to satisfy the boundary conditions.

In this paper, since no boundary equations are imposed for the free-free SWCNTs, $p$ is taken equal to zero. Moreover, a specific convergence analysis is carried out in order to select the degree of the Chebyshev polynomials: degree 11 is found suitably accurate $\left(M_{u}=M_{v}=M_{w}=11\right)$. Therefore, in the present paper, it is imposed $N_{p}=36$.

For a multi-mode analysis including different values of nodal diameters $n$, the number of degrees of freedom of the system is computed by the relation $N_{\max }=N_{p} \times(N+1)$, where $N$ represents the maximum value of the nodal diameters $n$ considered.

Equations (14) are inserted into the expressions of elastic strain energy (9) and kinetic energy (12) to compute the Rayleigh quotient $R(\tilde{\boldsymbol{q}})$, where $\tilde{\boldsymbol{q}}$ is a vector containing the unknown coefficients of expansions (15-17) [46]

$$
\tilde{\boldsymbol{q}}=\left[\begin{array}{c}
\vdots \\
\tilde{U}_{m, m} \\
\tilde{V}_{m, n} \\
\tilde{W}_{m, n} \\
\vdots
\end{array}\right]
$$


After imposing the stationarity to the Rayleigh quotient, the eigenvalue problem is obtained [46]

$$
\left(-\omega^{2} \tilde{\boldsymbol{M}}+\tilde{\boldsymbol{K}}\right) \tilde{\boldsymbol{q}}=\mathbf{0}
$$

which furnishes approximate natural frequencies (eigenvalues $\omega_{j}$ ) and mode shapes (eigenvectors $\left.\tilde{\boldsymbol{q}}_{j}\right)$, with $j=\left(1,2, \ldots, N_{\max }\right)$.

The approximated mode shape of the $j$-th mode is given by the equations (15-17) where coefficients $\left(\tilde{U}_{m, n}, \tilde{V}_{m . n}, \tilde{W}_{m, n}\right)$ are substituted with $\left(\tilde{U}_{m, n}^{(j)}, \tilde{V}_{m, n}^{(j)}, \tilde{W}_{m, n}^{(j)}\right)$, which denote the components of the $j$-th eigenvector $\tilde{\boldsymbol{q}}_{j}$ of the equation (24).

The approximation of the $j$-th eigenfunction vector of the original problem is given by [46]

$$
\tilde{\boldsymbol{W}}^{(j)}(\eta, \theta)=\left[\begin{array}{c}
\tilde{U}^{(j)}(\eta, \theta) \\
\tilde{V}^{(j)}(\eta, \theta) \\
\tilde{W}^{(j)}(\eta, \theta)
\end{array}\right]
$$

The $j$-th eigenfunction vector (25) can be normalized, as shown for example in Ref. [46], imposing the following

$$
\max \left[\max \left[U^{(j)}(\eta, \theta)\right], \max \left[V^{(j)}(\eta, \theta)\right], \max \left[W^{(j)}(\eta, \theta)\right]\right]=1
$$

The previous normalization has the following simple physical meaning. Suppose that the dominant direction of vibration of the $j$-th mode shape is along the thickness $h$ of the shell (radial direction $w$ ); then, the third component of the $j$-th eigenfunction vector (25) has maximum amplitude higher than the other two components. Therefore, if the $j$-th eigenfunction vector is normalised in order to obtain a maximum amplitude equal to unity, then the dimensionless time law (modal coordinate) associated to the third component of the $j$-th mode shape gives the maximum amplitude of vibration along the radial direction of the cylindrical shell. This normalization results to be particularly useful in the nonlinear vibration analysis, where the nonlinearity appears when the amplitude of the radial vibration is of the order of the thickness, i.e., the dimensionless amplitude is of the order of 1; in such situation, all Lagrangian variables are of the order of 1 or less, and this strongly improves the numerical accuracy and efficiency. 


\subsection{Nonlinear vibration analysis}

In the nonlinear vibration analysis, the full expression of the elastic strain energy (9) (quartic terms leading to cubic nonlinearity) is applied and the same procedure of Refs. [55,57] is followed. The three dimensionless displacements $\tilde{u}(\eta, \theta, \tau), \tilde{v}(\eta, \theta, \tau), \tilde{w}(\eta, \theta, \tau)$ are re-expanded by using the linear mode shapes $\tilde{U}^{(j, n)}(\eta, \theta), \tilde{V}^{(j, n)}(\eta, \theta), \tilde{W}^{(j, n)}(\eta, \theta)$ and their conjugates $\tilde{U}_{c}^{(j, n)}(\eta, \theta)$, $\tilde{V}_{c}^{(j, n)}(\eta, \theta), \tilde{W}_{c}^{(j, n)}(\eta, \theta)$, which are now available from the modal analysis of the previous section

$$
\begin{gathered}
\tilde{u}(\eta, \theta, \tau)=\sum_{j=1}^{N_{u}} \sum_{n=1}^{N}\left[\tilde{U}^{(j, n)}(\eta, \theta) \tilde{f}_{u, j, n}(t)+\tilde{U}_{c}^{(j, n)}(\eta, \theta) \tilde{f}_{u, j, n, c}(t)\right] \\
\tilde{v}(\eta, \theta, \tau)=\sum_{j=1}^{N_{v}} \sum_{n=1}^{N}\left[\tilde{V}^{(j, n)}(\eta, \theta) \tilde{f}_{v, j, n}(\tau)+\tilde{V}_{c}^{(j, n)}(\eta, \theta) \tilde{f}_{v, j, n, c}(\tau)\right] \\
\tilde{w}(\eta, \theta, \tau)=\sum_{j=1}^{N_{w}} \sum_{n=1}^{N}\left[\tilde{W}^{(j, n)}(\eta, \theta) \tilde{f}_{w, j, n}(\tau)+\tilde{W}_{c}^{(j, n)}(\eta, \theta) \tilde{f}_{w, j, n, c}(\tau)\right]
\end{gathered}
$$

where the dimensionless time laws $\left(\tilde{f}_{u, j, n}(\tau), \tilde{f}_{u, j, n, c}(\tau), \tilde{f}_{v, j, n}(\tau), \tilde{f}_{v, j, n, c}(\tau), \tilde{f}_{w, j, n}(\tau), \tilde{f}_{w, j, n, c}(\tau)\right)$ are unknown functions, the index $j$ is used for ordering the modes with increasing natural frequency and the index $n$ indicates the number of nodal diameters of the mode shape of the shell.

From expansions (27-29), it can be observed that, in the nonlinear analysis, the time synchronicity is relaxed: for each mode $j$ and for each component $(\tilde{u}, \tilde{v}, \tilde{w})$ different time laws $\tilde{f}(\tau)$ are allowed.

\subsection{Lagrange equations}

The expansions (27-29) are inserted into the equations of dimensionless elastic strain energy (9) and kinetic energy (12); the dimensionless Lagrange equations of motion, in the case of free vibrations, can be expressed in the form

$$
\frac{d}{d \tau}\left(\frac{\partial \tilde{T}}{\partial \tilde{q}_{i}^{\prime}}\right)+\frac{\partial \tilde{U}}{\partial \tilde{q}_{i}}=0 \quad i \in\left[1, N_{\max }\right]
$$

where the maximum number of degrees of freedom $N_{\max }$ depends on the number of vibration modes considered in the expansions (27-29). 
The dimensionless lagrangian coordinates $\left(\tilde{q}_{i}, \tilde{q}_{i}^{\prime}, \tilde{q}_{i}^{\prime \prime}\right)$ can be written as

$$
\tilde{q}_{i}=\frac{q_{i}}{R} \quad \tilde{q}_{i}^{\prime}=\frac{d \tilde{q}_{i}}{d \tau}=\frac{\dot{q}_{i}}{\omega_{0} R} \quad \tilde{q}_{i}^{\prime \prime}=\frac{d \tilde{q}_{i}^{\prime}}{d \tau}=\frac{\ddot{q}_{i}}{\omega_{0}^{2} R} \quad i \in\left[1, N_{\max }\right]
$$

where $\tilde{q}_{i}$ correspond to the previous dimensionless modal coordinates $\left(\tilde{f}_{u, j, n}(\tau), \tilde{f}_{u, j, n, c}(\tau), \tilde{f}_{v, j, n}(\tau)\right.$, $\left.\tilde{f}_{v, j, n, c}(\tau), \tilde{f}_{w, j, n}(\tau), \tilde{f}_{w, j, n, c}(\tau)\right)$ and $\left(q_{i}, \dot{q}_{i}, \ddot{q}_{i}\right)$ are the dimensional lagrangian coordinates.

By substituting the dimensionless vector functions $\tilde{\mathbf{F}}\left(\tilde{q}_{i}\right)=\partial \tilde{U} / \partial \tilde{q}_{i}$ and $\tilde{\mathbf{M}} \tilde{q}_{i}^{\prime \prime}=d\left(\partial \tilde{T} / \partial \tilde{q}_{i}^{\prime}\right) / d \tau$ into equation (30), where $\tilde{M}$ is the dimensionless mass matrix, it is obtained (see Ref. [57])

$$
\tilde{\mathbf{M}} \tilde{q}_{i}^{\prime \prime}+\tilde{\mathbf{F}}\left(\tilde{q}_{i}\right)=\mathbf{0} \quad i \in\left[1, N_{\max }\right]
$$

Introducing the vector function $\tilde{\mathbf{F}}_{\mathbf{x}, \mathbf{i}}=\tilde{\mathbf{M}}^{-1} \tilde{\mathbf{F}}\left(\tilde{q}_{i}\right)$ into the equation (32), the equations of motion for free vibrations can be expressed in the following form

$$
\tilde{q}_{i}^{\prime \prime}+\tilde{F}_{x, i}=0 \quad i \in\left[1, N_{\max }\right]
$$

The set of nonlinear ordinary differential equations (33), completed by the modal initial conditions on displacements and velocities, is then solved numerically by means of the implicit Runge-Kutta iterative method with properly accuracy, precision and number of steps. 


\section{Numerical results}

The numerical analyses carried out in this section are obtained considering equivalent mechanical parameters listed in Table 1 (see Ref. [41]).

In Tables 2-3, the vibrational approach adopted in this paper, based on the Sanders-Koiter thin shell theory and the equivalent mechanical parameters reported in Table 1, is validated in the linear field (natural frequencies) by means of comparisons with experimental studies and numerical simulations from the pertinent literature. From these comparisons, it can be observed that the present continuous model is in very good agreement with the results of RRS [11] and MDS [17].

It must be underlined that this validation in linear field confirms the correctness of the continuous shell model proposed in the present paper with regard to the choice of the values of the equivalent mechanical parameters and to the previously assumed hypotheses (no size effects, isotropic shell). In Table 4 and Figures 2-4, natural frequencies and mode shapes of the free-free SWCNT of Table 1 with radius $R=0.39 \mathrm{~nm}$ and length $L=3.0 \mathrm{~nm}$ obtained by applying the Sanders-Koiter thin shell theory are reported.

In the following, a further index is used for defining a mode shape and its longitudinal feature, i.e. the index $m$, which indicates the number of nodal circumferences. It should be reminded that the mode shapes with $(m=0)$ (no longitudinal half-waves) and $(m=1)$ (one-half longitudinal halfwave) of Figure 4 correspond to the Rayleigh and the Love inextensional modes, respectively [39]. In Figures 5-6, mode shapes of four different pairs of conjugate modes of the free-free SWCNT of Table 1 having radius $R=0.39 \mathrm{~nm}$ and length $L=3.0 \mathrm{~nm}$ are reported; the conjugate modes have the same natural frequency and mode shape angularly shifted by $\pi / 2 n$. In particular, the conjugate modes $(m, n)=(1,2),(m, n)=(1,2, c)$ with natural frequency $f=1.21558 \mathrm{THz}$ and the conjugate modes $(m, n)=(3,2),(m, n)=(3,2, c)$ with natural frequency $f=2.32386 \mathrm{THz}$ have the respective mode shapes rotated by $\pi / 4$, while the conjugate modes $(m, n)=(1,4),(m, n)=(1,4, c)$ with natural frequency $f=6.42757 \mathrm{THz}$ and the conjugate modes $(m, n)=(3,4),(m, n)=(3,4, c)$ with natural frequency $f=6.9507 \mathrm{THz}$ have the respective mode shapes rotated by $\pi / 8$.

\begin{tabular}{|c|c|}
\hline Young's modulus $E[\mathrm{TPa}]$ & 5.5 \\
\hline Poisson's ratio $v$ & 0.19 \\
\hline Mass density $\rho\left[\mathrm{kg} / \mathrm{m}^{3}\right]$ & 11700 \\
\hline Thickness $h[\mathrm{~nm}]$ & 0.066 \\
\hline
\end{tabular}

Table 1. Equivalent mechanical parameters of the free-free SWCNT [41]. 
Nonlinear resonance interaction between conjugate circumferential flexural modes in single-walled carbon nanotubes

\begin{tabular}{|c|c|c|c|c|}
\hline Radius & Aspect ratio & \multicolumn{2}{|c|}{ Natural frequency $(\mathrm{THz})$} & Difference $\%$ \\
\hline$R[\mathrm{~nm}]$ & $L / D$ & SKT - Present model & $\mathrm{RRS}-[11]$ & \\
\hline 0.509 & 10 & 6.905 & 7.165 & 3.63 \\
\hline 0.518 & 10 & 6.785 & 7.105 & 4.50 \\
\hline 0.527 & 10 & 6.669 & 6.865 & 2.85 \\
\hline 0.569 & 10 & 6.177 & 6.295 & 1.87 \\
\hline 0.705 & 10 & 5.025 & 5.276 & 4.76 \\
\hline 0.708 & 10 & 4.964 & 5.216 & 4.83 \\
\hline 0.718 & 10 & 4.895 & 5.066 & 3.37 \\
\hline 0.733 & 10 & 4.788 & 4.947 & 3.21 \\
\hline 0.746 & 10 & 4.711 & 4.917 & 4.19 \\
\hline 0.764 & 10 & 4.594 & 4.797 & 4.23 \\
\hline 0.770 & 10 & 4.559 & 4.737 & 3.76 \\
\hline 0.782 & 10 & 4.494 & 4.677 & 3.91 \\
\hline 0.799 & 10 & 4.393 & 4.617 & 4.85 \\
\hline 0.814 & 10 & 4.318 & 4.527 & 4.62 \\
\hline 0.822 & 10 & 4.271 & 4.437 & 3.74 \\
\hline 0.847 & 10 & 4.150 & 4.317 & 4.04 \\
\hline
\end{tabular}

Table 2. Natural frequencies of the radial breathing mode $(m=0, n=0)$ of the free-free SWCNT of Table 1 for different values of radius $R$ and same value of aspect ratio $L / D$. Comparisons between Sanders-Koiter elastic shell theory (SKT) (present model) and experimental resonant Raman spectroscopy (RRS) [11]. 
Nonlinear resonance interaction between conjugate circumferential flexural modes in single-walled carbon nanotubes

\begin{tabular}{|c|c|c|c|c|}
\hline Radius & Aspect ratio & \multicolumn{2}{|c|}{ Natural frequency $(\mathrm{THz})$} & Difference $\%$ \\
\hline$R[\mathrm{~nm}]$ & $L / D$ & SKT - Present model & MDS - [17] & \\
\hline 0.339 & 5.26 & 0.217 & 0.212 & 2.36 \\
\hline 0.339 & 5.62 & 0.191 & 0.188 & 1.60 \\
\hline 0.339 & 5.99 & 0.169 & 0.167 & 1.20 \\
\hline 0.339 & 6.35 & 0.151 & 0.150 & 0.67 \\
\hline 0.339 & 6.71 & 0.136 & 0.136 & $\leq 0.01$ \\
\hline 0.339 & 7.07 & 0.123 & 0.123 & $\leq 0.01$ \\
\hline 0.339 & 7.44 & 0.112 & 0.112 & $\leq 0.01$ \\
\hline 0.339 & 7.80 & 0.102 & 0.102 & $\leq 0.01$ \\
\hline 0.339 & 8.16 & 0.094 & 0.094 & $\leq 0.01$ \\
\hline 0.339 & 8.52 & 0.086 & 0.086 & $\leq 0.01$ \\
\hline 0.339 & 8.89 & 0.080 & 0.080 & $\leq 0.01$ \\
\hline 0.339 & 9.25 & 0.074 & 0.074 & $\leq 0.01$ \\
\hline 0.339 & 9.61 & 0.069 & 0.069 & $\leq 0.01$ \\
\hline 0.339 & 9.98 & 0.064 & 0.064 & $\leq 0.01$ \\
\hline 0.339 & 10.34 & 0.060 & 0.060 & $\leq 0.01$ \\
\hline 0.339 & 10.70 & 0.056 & 0.056 & $\leq 0.01$ \\
\hline 0.339 & 11.06 & 0.053 & 0.053 & $\leq 0.01$ \\
\hline
\end{tabular}

Table 3. Natural frequencies of the beam-like mode $(m=0, n=1)$ of the clamped-free SWCNT of Table 1 for the same value of the radius $R$ and different values of the aspect ratio $L / D$. Comparisons between Sanders-Koiter elastic thin shell theory (SKT) (present model) and numerical molecular dynamics simulations (MDS) [17]. 
Nonlinear resonance interaction between conjugate circumferential flexural modes in single-walled carbon nanotubes

\begin{tabular}{|c|c|}
\hline Vibration mode $(m, n)$ & Natural frequency [THz] \\
\hline$(0,2)$ & 1.17609 \\
\hline$(1,2)$ & 1.21558 \\
\hline$(2,2)$ & 1.52195 \\
\hline$(3,2)$ & 2.32386 \\
\hline$(0,4)$ & 6.37264 \\
\hline$(1,4)$ & 6.42757 \\
\hline$(2,4)$ & 6.61777 \\
\hline$(3,4)$ & 6.95070 \\
\hline$(0,0)$ & 9.04466 \\
\hline$(1,0)$ & 9.28746 \\
\hline$(2,0)$ & 8.60968 \\
\hline$(3,0)$ & 8.79534 \\
\hline
\end{tabular}

Table 4. Natural frequencies of the free-free SWCNT of Table 1 with radius $R=0.39 \mathrm{~nm}$ and length $L=3.0 \mathrm{~nm}$. 
Nonlinear resonance interaction between conjugate circumferential flexural modes in single-walled carbon nanotubes

Mode $(m, n)=(0,2)$

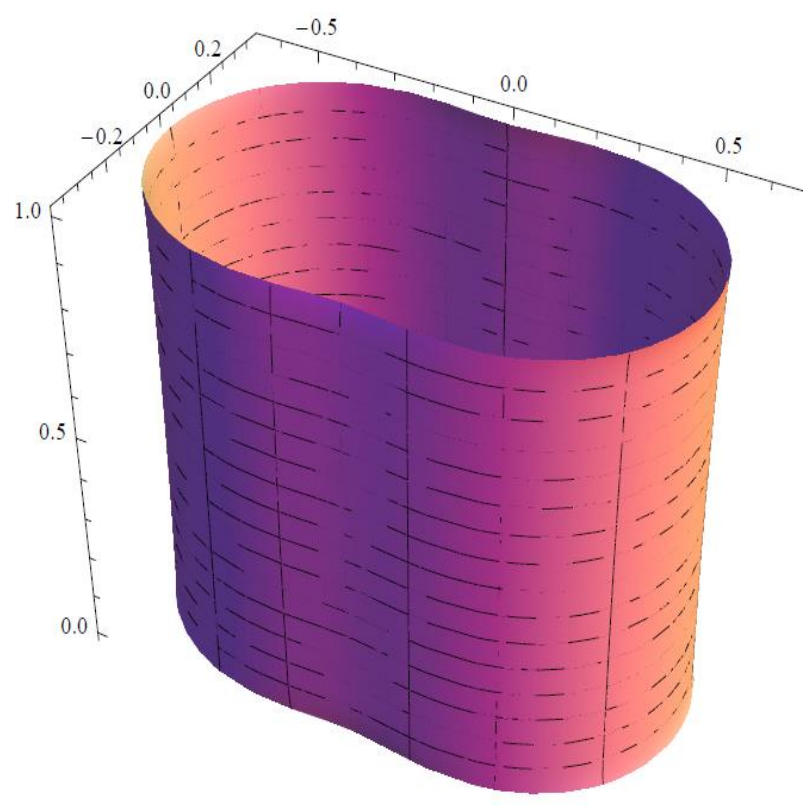

Mode $(m, n)=(2,2)$

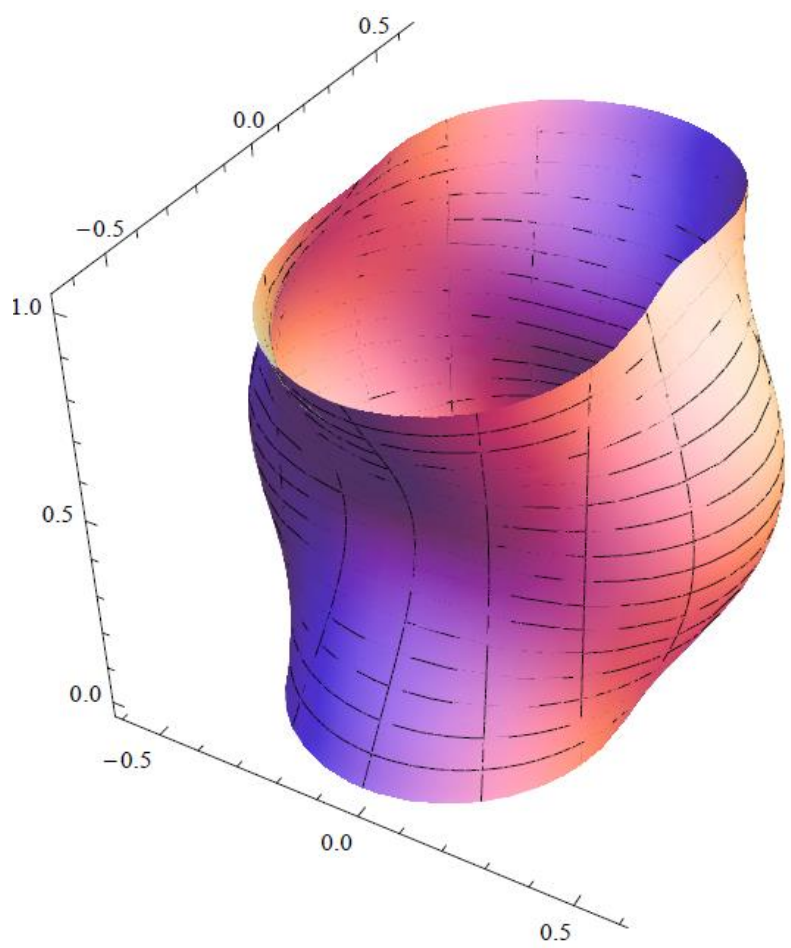

Mode $(m, n)=(1,2)$

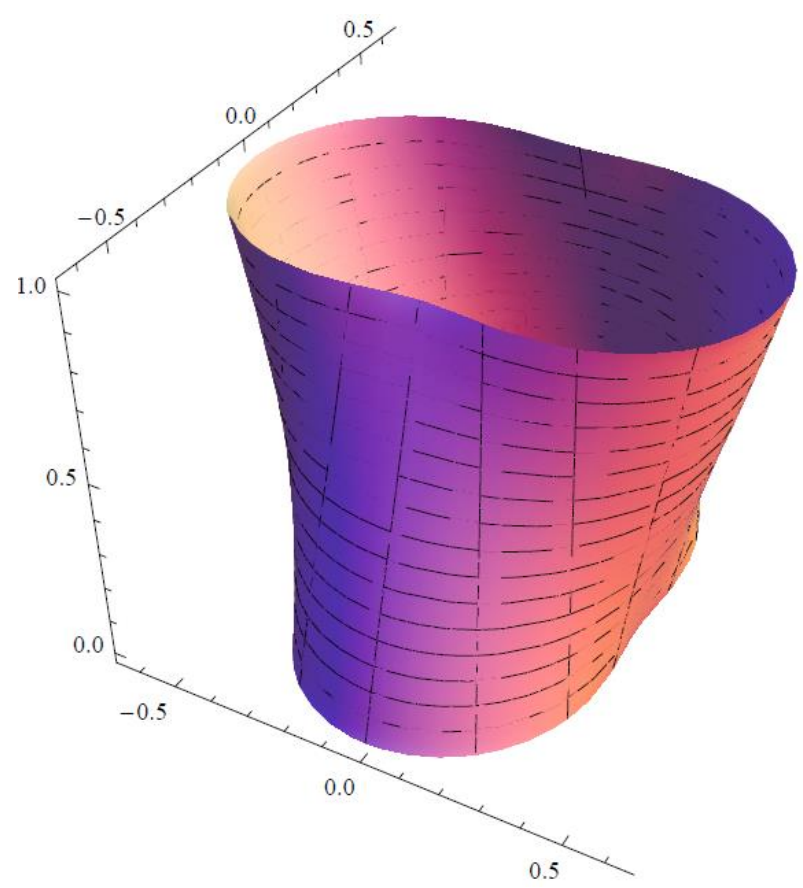

Mode $(m, n)=(3,2)$

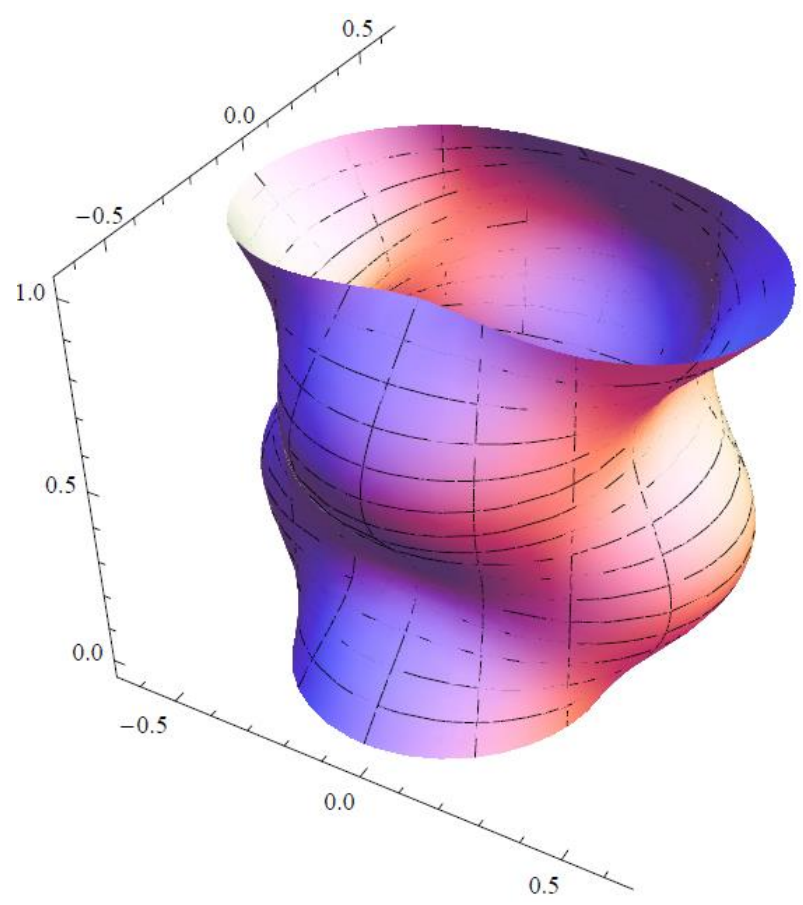

Figure 2. Mode shapes of the free-free SWCNT of Table 1 with radius $R=0.39 \mathrm{~nm}$ and length $L=3.0 \mathrm{~nm}$. Mode $(m, n)=(0,2)$. Mode $(m, n)=(1,2)$. Mode $(m, n)=(2,2)$. Mode $(m, n)=(3,2)$. 
Nonlinear resonance interaction between conjugate circumferential flexural modes in single-walled carbon nanotubes

Mode $(m, n)=(0,4)$

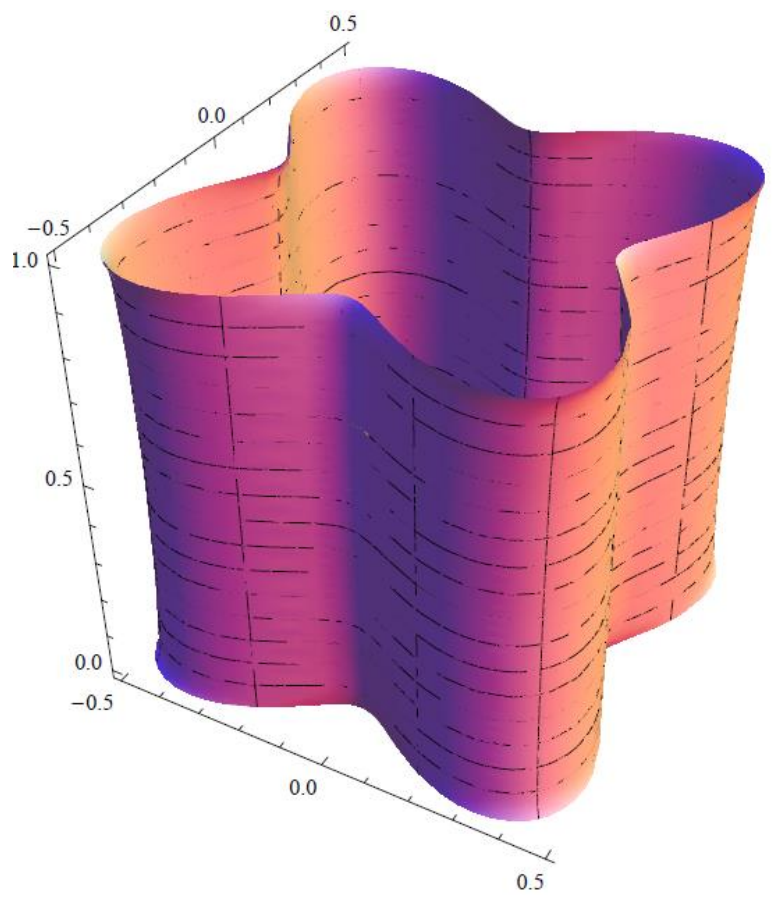

Mode $(m, n)=(2,4)$

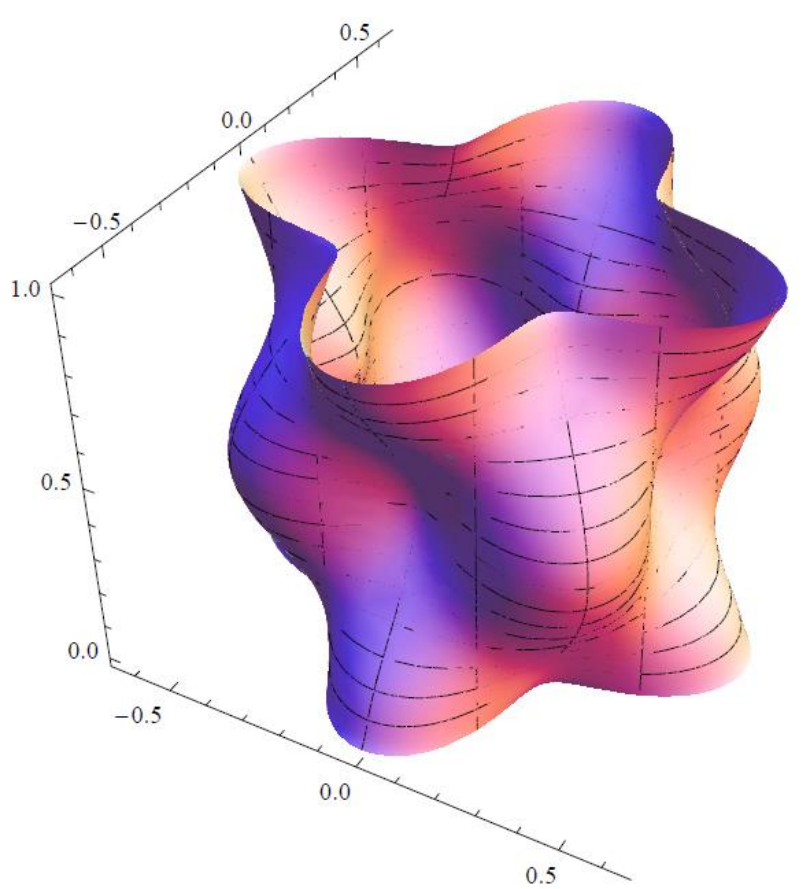

Mode $(m, n)=(1,4)$

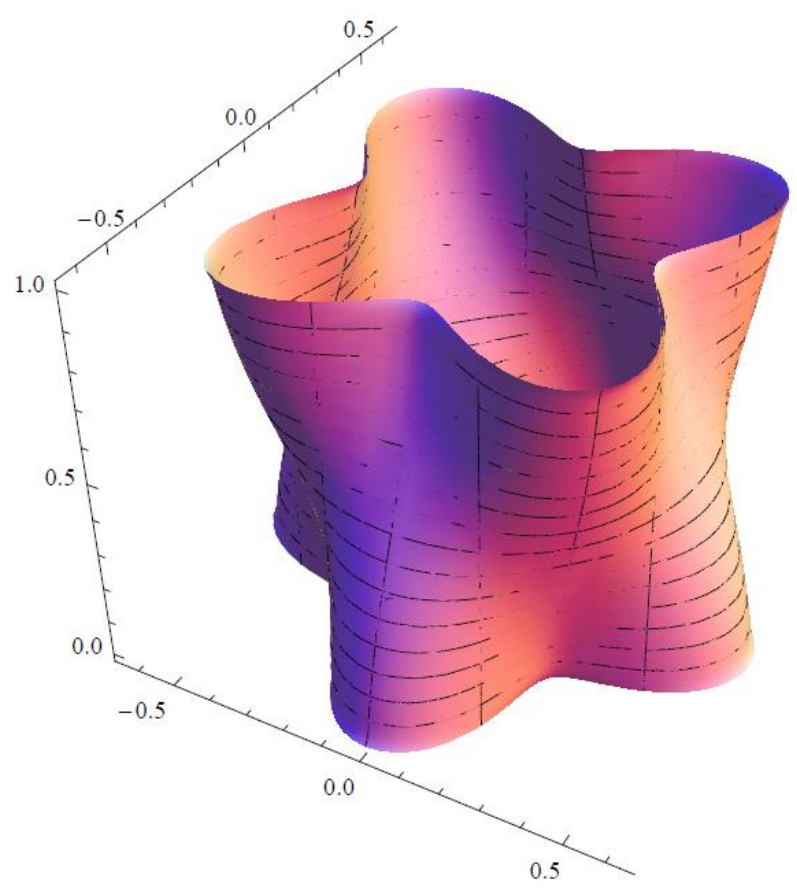

Mode $(m, n)=(3,4)$

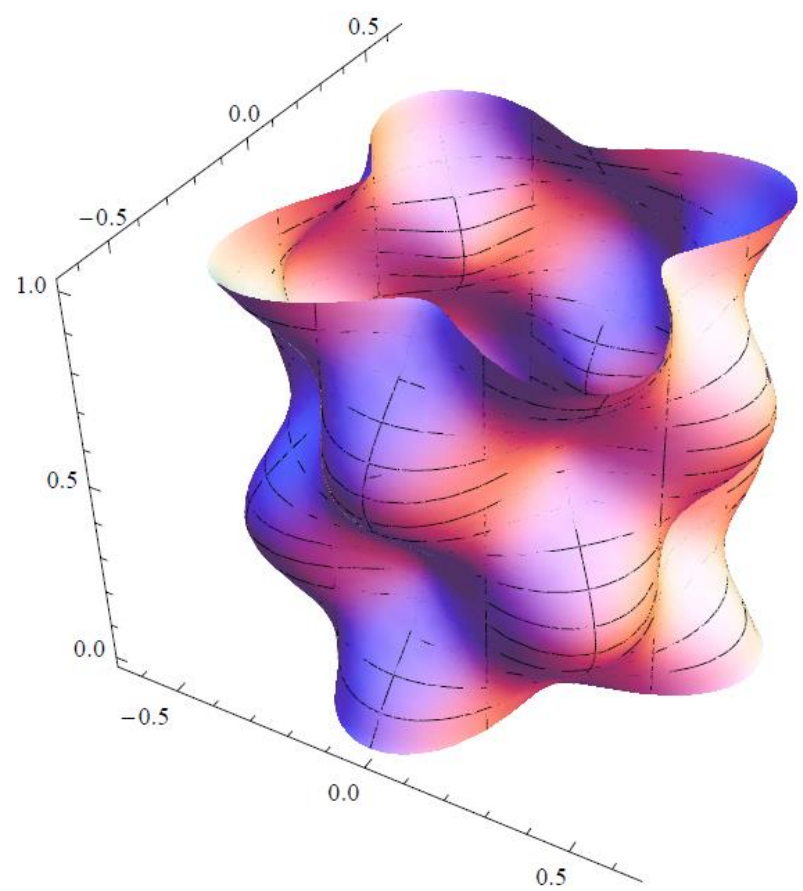

Figure 3. Mode shapes of the free-free SWCNT of Table 1 with radius $R=0.39 \mathrm{~nm}$ and length $L=3.0 \mathrm{~nm}$. Mode $(m, n)=(0,4)$. Mode $(m, n)=(1,4)$. Mode $(m, n)=(2,4)$. Mode $(m, n)=(3,4)$. 
Nonlinear resonance interaction between conjugate circumferential flexural modes in single-walled carbon nanotubes

Mode $(m, n)=(0,0)$

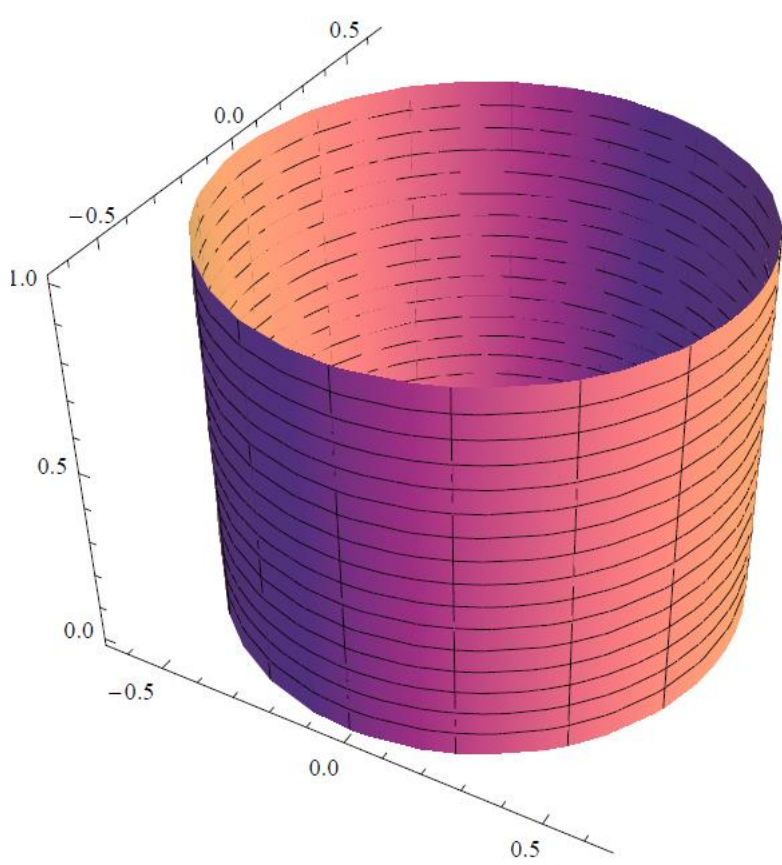

Mode $(m, n)=(2,0)$

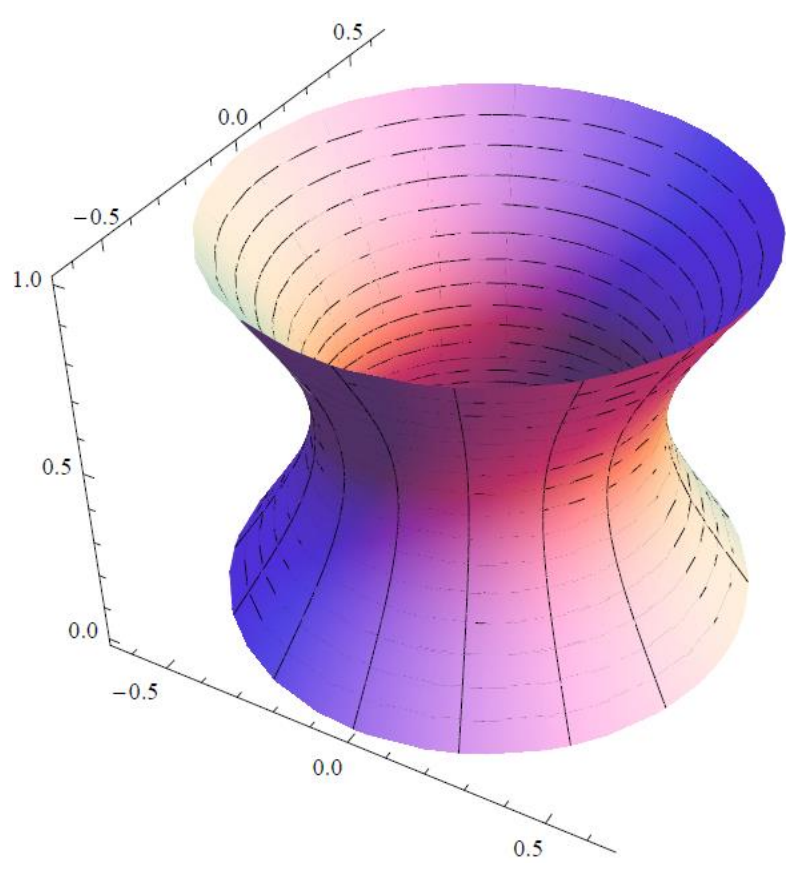

Mode $(m, n)=(1,0)$

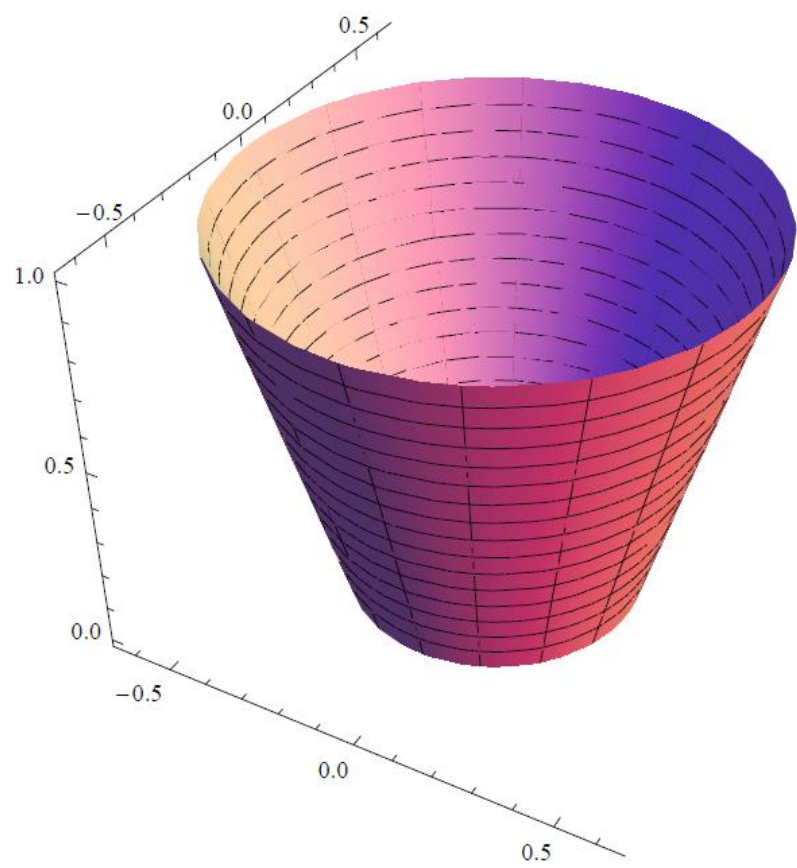

Mode $(m, n)=(3,0)$

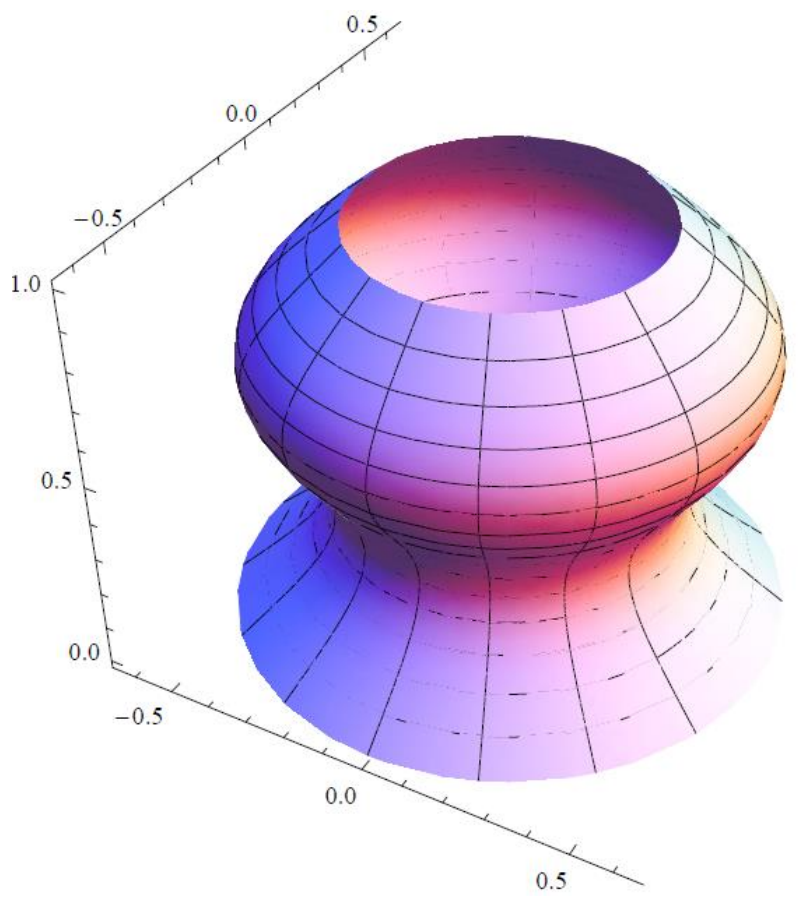

Figure 4. Mode shapes of the free-free SWCNT of Table 1 with radius $R=0.39 \mathrm{~nm}$ and length $L=3.0 \mathrm{~nm}$. Rayleigh inextensional mode $(m, n)=(0,0)$, Love inextensional mode $(m, n)=(1,0)[39]$. Mode $(m, n)=(2,0)$. Mode $(m, n)=(3,0)$. 
Nonlinear resonance interaction between conjugate circumferential flexural modes in single-walled carbon nanotubes

Mode $(m, n)=(1,2)$

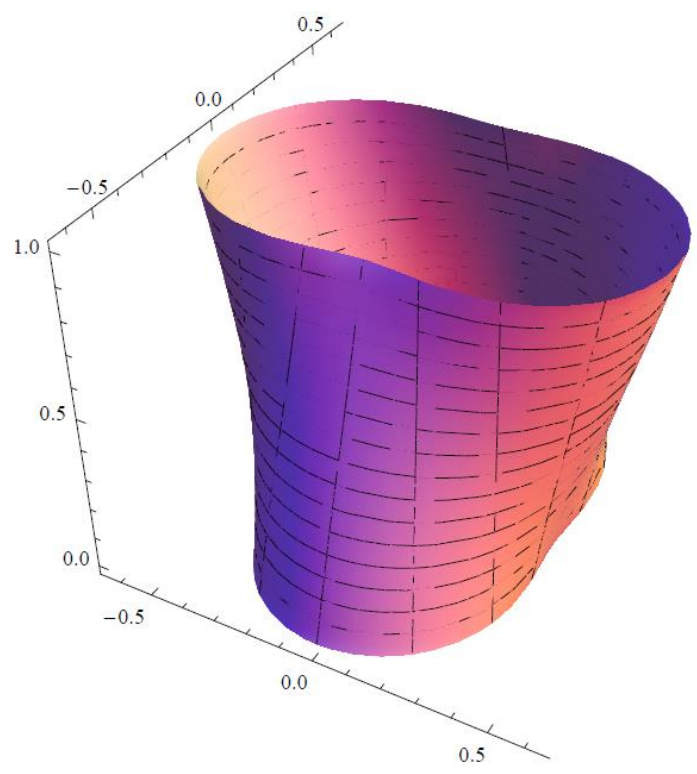

Mode $(m, n)=(3,2)$

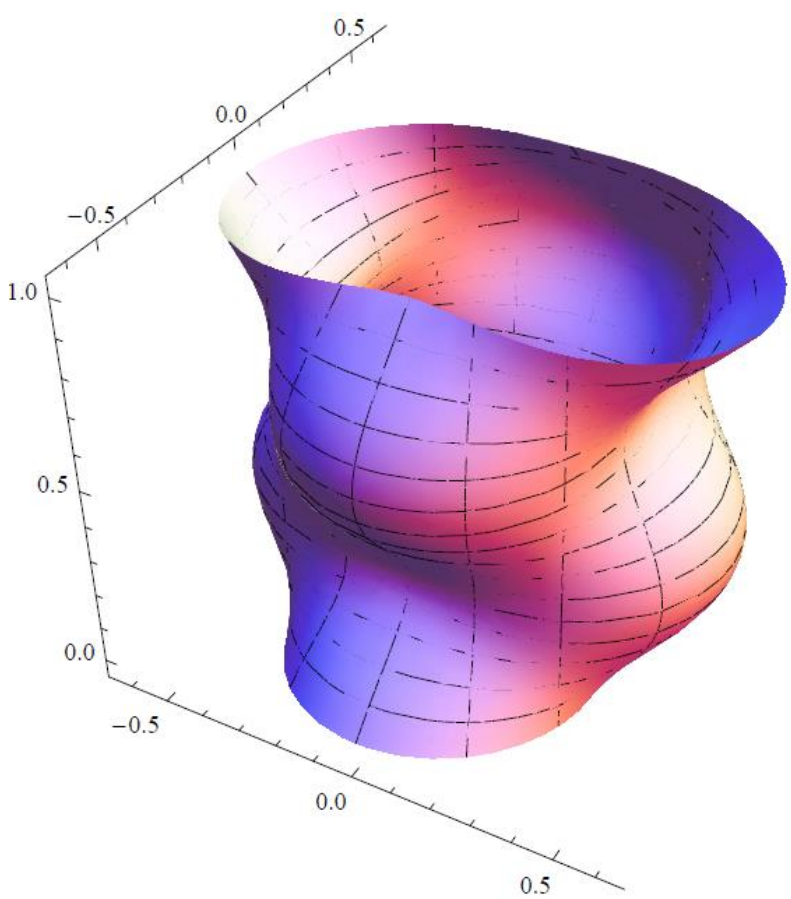

Mode $(m, n)=(1,2, c)$

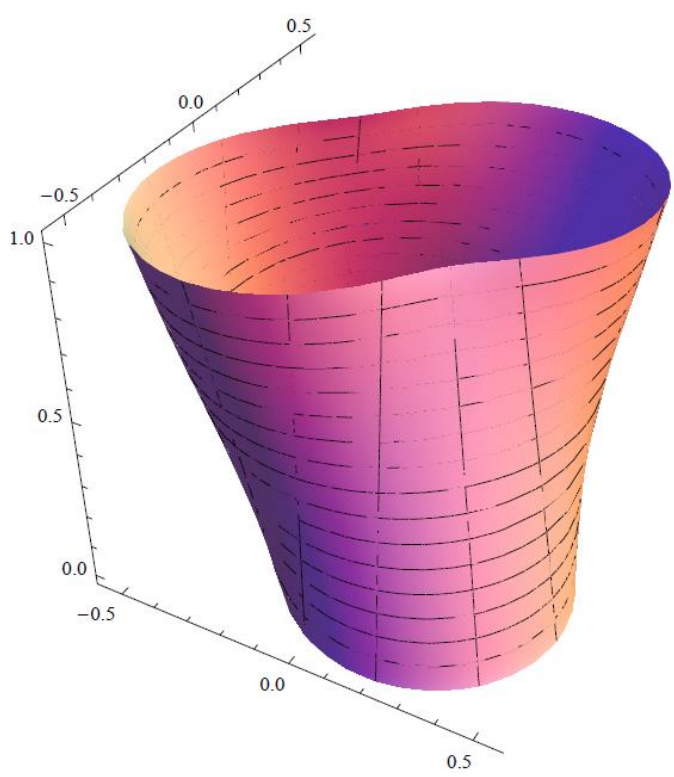

Mode $(m, n)=(3,2, c)$

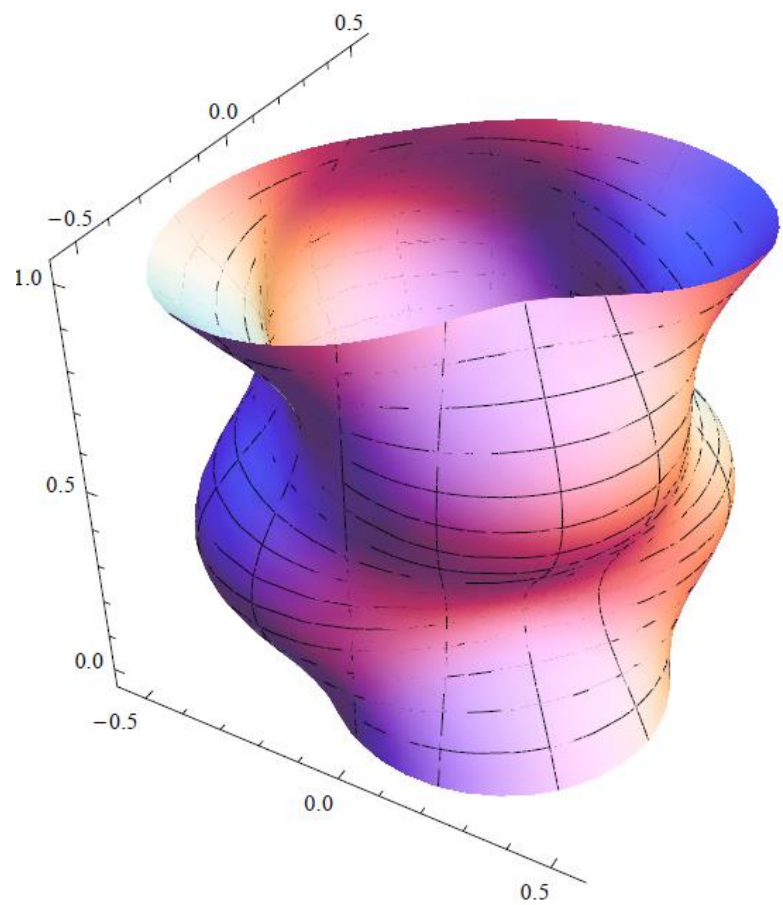

Figure 5. Mode shape comparisons of the free-free SWCNT of Table 1 with radius $R=0.39 \mathrm{~nm}$ and length $L=3.0 \mathrm{~nm}$.

Conjugate modes $(m, n)=(1,2),(m, n)=(1,2, c)$ : natural frequency $f=1.21558 \mathrm{THz}$, mode shapes rotated by $\pi / 4$.

Conjugate modes $(m, n)=(3,2),(m, n)=(3,2, c)$ : natural frequency $f=2.32386 \mathrm{THz}$, mode shapes rotated by $\pi / 4$. 
Mode $(m, n)=(1,4)$

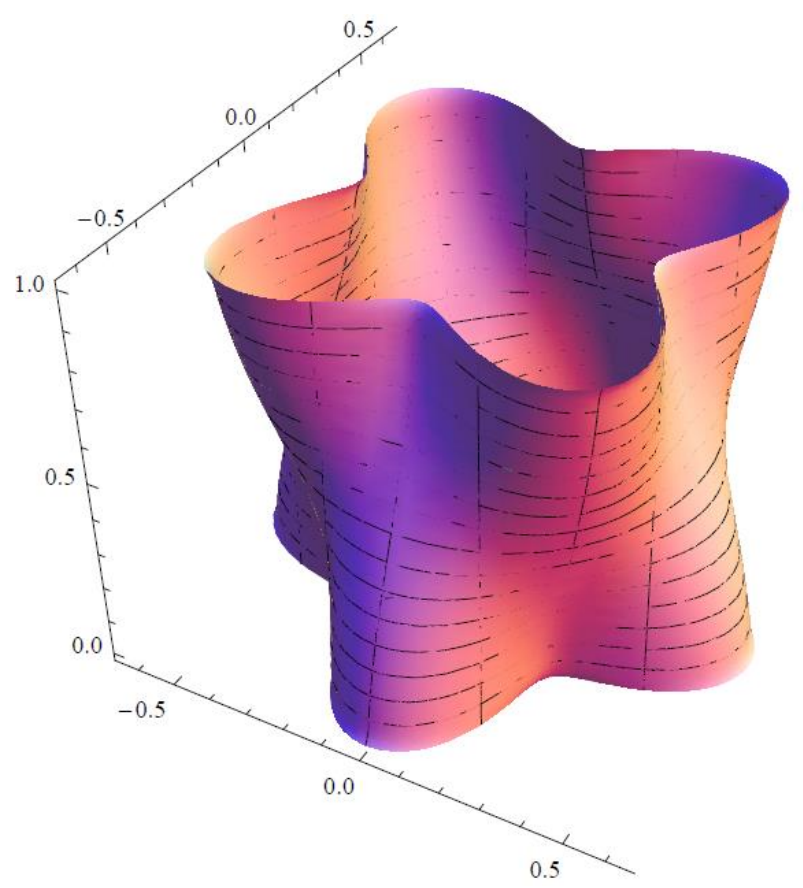

Mode $(m, n)=(3,4)$

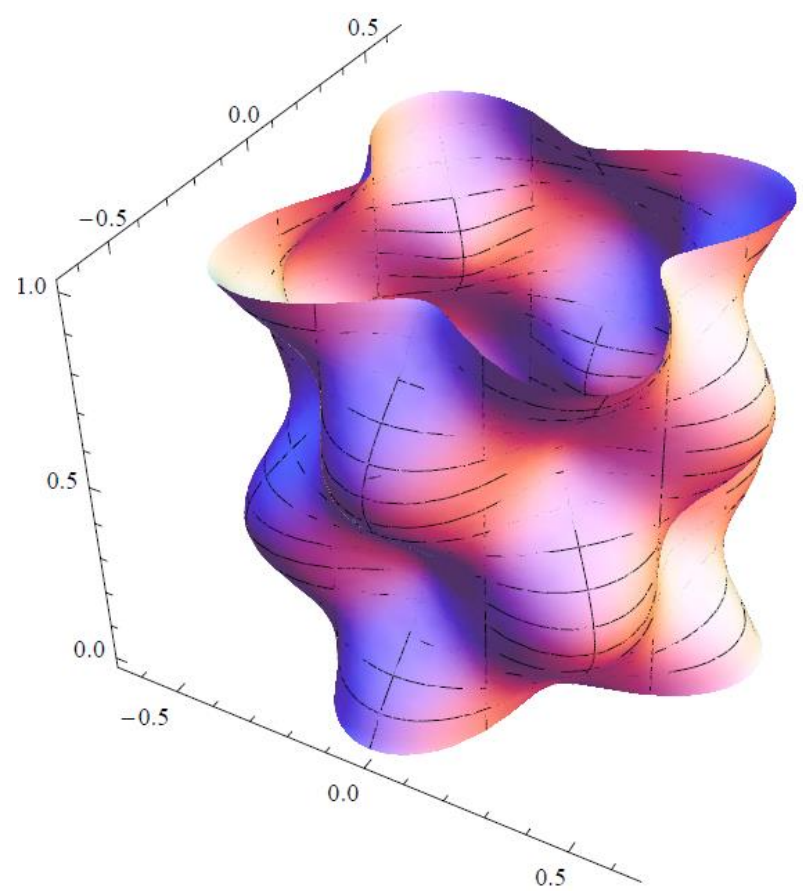

Mode $(m, n)=(1,4, c)$

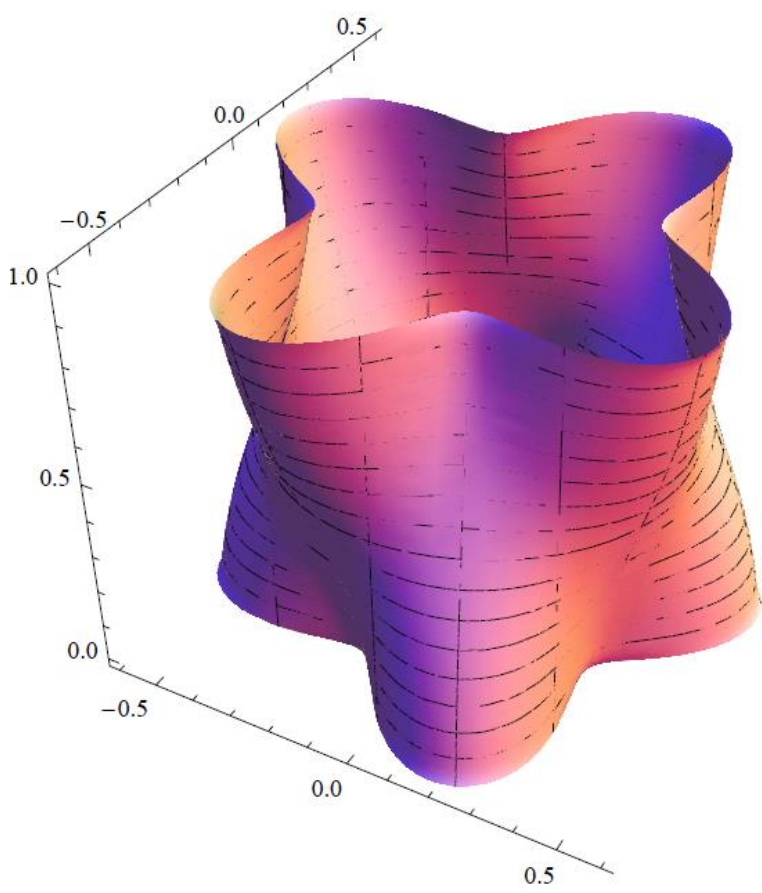

Mode $(m, n)=(3,4, c)$

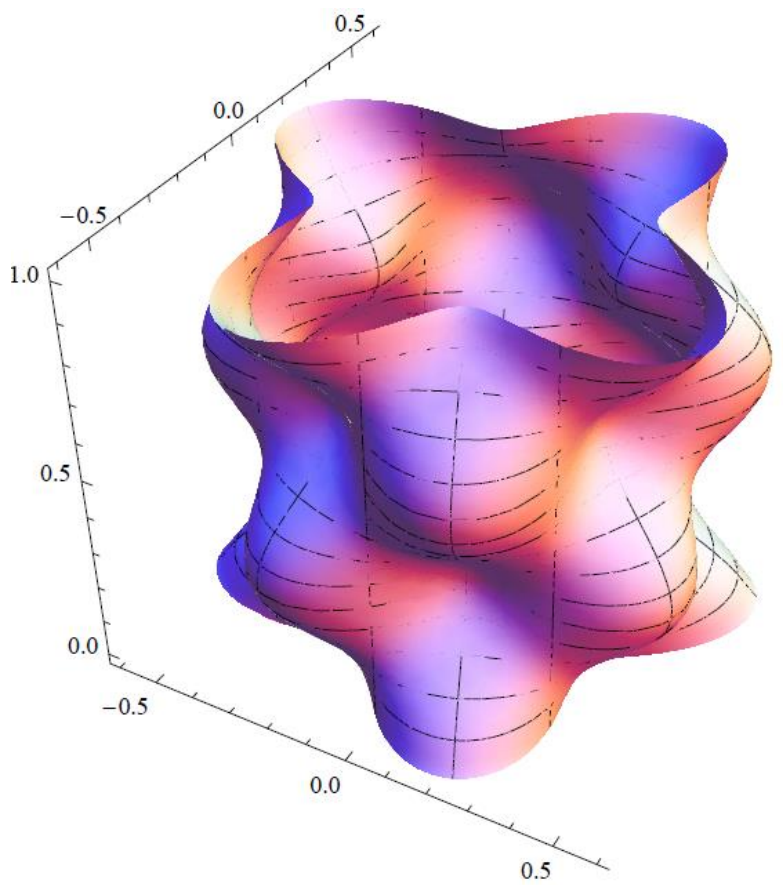

Figure 6. Mode shape comparisons of the free-free SWCNT of Table 1 with radius $R=0.39 \mathrm{~nm}$ and length $L=3.0 \mathrm{~nm}$.

Conjugate modes $(m, n)=(1,4),(m, n)=(1,4, c)$ : natural frequency $f=6.42757 \mathrm{THz}$, mode shapes rotated by $\pi / 8$.

Conjugate modes $(m, n)=(3,4),(m, n)=(3,4, c)$ : natural frequency $f=6.95070 \mathrm{THz}$, mode shapes rotated by $\pi / 8$. 


\subsection{Linear analysis}

In order to better understand the nonlinear effect of the resonance interaction between the conjugate CFMs considered, an initial linear study is carried out by taking into account only the linear terms of the dimensionless middle surface strains (2-4) into the expression of the dimensionless elastic strain energy (9).

The three displacements $\tilde{u}(\eta, \theta, \tau), \tilde{v}(\eta, \theta, \tau), \tilde{w}(\eta, \theta, \tau)$ are expanded considering the approximated linear mode shapes $\tilde{U}^{(j, n)}(\eta, \theta), \tilde{U}^{(j, n, c)}(\eta, \theta), \tilde{V}^{(j, n)}(\eta, \theta), \tilde{V}^{(j, n, c)}(\eta, \theta), \tilde{W}^{(j, n)}(\eta, \theta), \tilde{W}^{(j, n, c)}(\eta, \theta)$ of the two conjugate modes $(1,2),(1,2, c)$ and the following linear modal expansion is obtained

$$
\begin{gathered}
\tilde{u}(\eta, \theta, \tau)=\tilde{U}^{(1,2)}(\eta, \theta) \tilde{f}_{u, 1,2}(\tau)+\tilde{U}^{(1,2, c)}(\eta, \theta) \tilde{f}_{u, 1,2, c}(\tau) \\
\tilde{v}(\eta, \theta, \tau)=\tilde{V}^{(1,2)}(\eta, \theta) \tilde{f}_{v, 1,2}(\tau)+\tilde{V}^{(1,2, c)}(\eta, \theta) \tilde{f}_{v, 1,2, c}(\tau) \\
\tilde{w}(\eta, \theta, \tau)=\tilde{W}^{(1,2)}(\eta, \theta) \tilde{f}_{w, 1,2}(\tau)+\tilde{W}^{(1,2, c)}(\eta, \theta) \tilde{f}_{w, 1,2, c}(\tau)
\end{gathered}
$$

where $\tilde{f}_{u, 1,2}(\tau), \tilde{f}_{u, 1,2, c}(\tau), \tilde{f}_{v, 1,2}(\tau), \tilde{f}_{v, 1,2, c}(\tau), \tilde{f}_{w, 1,2}(\tau), \tilde{f}_{w, 1,2, c}(\tau)$ are unknown linear modal coordinates and the maximum number of degrees of freedom is $N_{\max }=6$.

The modal initial conditions $\tilde{f}_{u, 1,2}(0), \tilde{f}_{u, 1,2, c}(0), \tilde{f}_{v, 1,2}(0), \tilde{f}_{v, 1,2, c}(0), \tilde{f}_{w, 1,2}(0), \tilde{f}_{w, 1,2, c}(0)$ imposed on the linear modal coordinates are given by

$$
\begin{array}{clrl}
\tilde{f}_{u, 1,2}(0)=0.1 & \tilde{f}_{v, 1,2}(0)=0.1 & \tilde{f}_{w, 1,2}(0)=0.1 \\
\tilde{f}_{u, 1,2, c}(0)=0.05 & \tilde{f}_{v, 1,2, c}(0)=0.05 & \tilde{f}_{w, 1,2, c}(0)=0.05
\end{array}
$$

It should be highlighted that, in the present analysis, the initial velocities are taken equal to zero (the modal initial conditions are imposed only on the displacements).

The system of linear ordinary differential equations of motion (33), completed with the initial conditions (35), is then solved through an implicit Runge-Kutta numerical method in order to obtain time histories, frequency spectra and phase portraits describing the linear steady-state dynamic response of the SWCNT. 


\subsubsection{Time histories, frequency spectra, phase portraits}

The natural frequency and dimensionless time period of the two conjugate modes $(1,2),(1,2, c)$ are $f_{(1,2)}=f_{(1,2, c)}=1.21558 \mathrm{THz}$ and $\tau_{(1,2)}=\tau_{(1,2, c)}=2 \times \pi \times f_{(0,2)} / f_{(1,2)}=6.07905$, respectively, where $f_{(0,2)}=$ $1.17609 \mathrm{THz}$ is the natural frequency of the reference (i.e. lowest frequency) mode $(0,2)$.

The time histories of the two conjugate modes $(1,2),(1,2, c)$ are reported in Figure 7 (a); this figure clearly proves that the vibration is a periodic motion, i.e. the CNT is vibrating in linear conditions. The corresponding frequency spectra are shown in Figure 7(b), where the horizontal axis represents the normalised circular frequency $\omega / \omega_{(0,2)}$. The spectra are perfectly clean, with a single spike at the frequency corresponding to the eigenfrequency of the selected mode, as it is expected in linear field. It must be stressed that these analyses were performed in order to compare the results of the linear vibrations with the following results of the nonlinear vibrations.

(a)

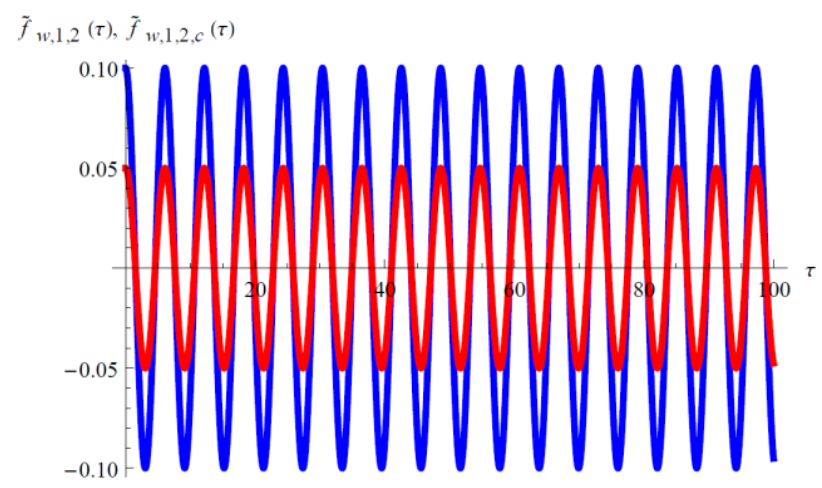

(b)

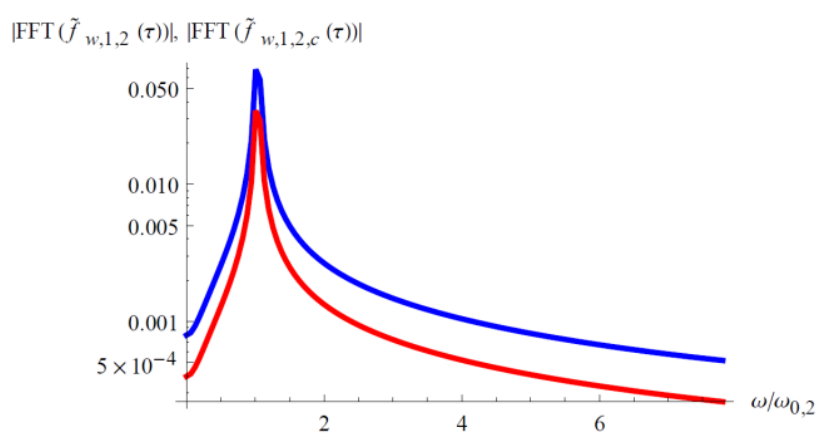

Figure 7. Time histories (a) and frequency spectra (b) of the two conjugate modes $(1,2),(1,2, c)$. Free-free SWCNT of Table 1. Modal expansion (34). Initial conditions (35). - Mode (1,2). - Mode $(1,2, c)$. Time period $\tau_{(1,2)}=6.07905$.

The two-dimensional phase portrait of the mode $(1,2)$ in the plane (displacement-velocity) is shown in Figure $8(\mathrm{a})$. By calculating the first (lowest) root of the equation $\tilde{f}_{w, 1,2}(\tau)=\tilde{f}_{w, 1,2}(0)=0.1$, the time $\tau=6.07905$ is found, which is equal to the time period $\tau_{(1,2)}$ (the motion is periodic).

Similarly, the two-dimensional phase portrait of the conjugate vibration mode $(1,2, c)$ into the plane (displacement-velocity) is reported in Figure 8(b). Calculating the first (lowest) root of the equation $\tilde{f}_{w, 1,2, c}(\tau)=\tilde{f}_{w, 1,2, c}(0)=0.05$, the same time $\tau=6.07905$ of the mode $(1,2)$ is obtained (the conjugate modes have the same time period). 
(a)

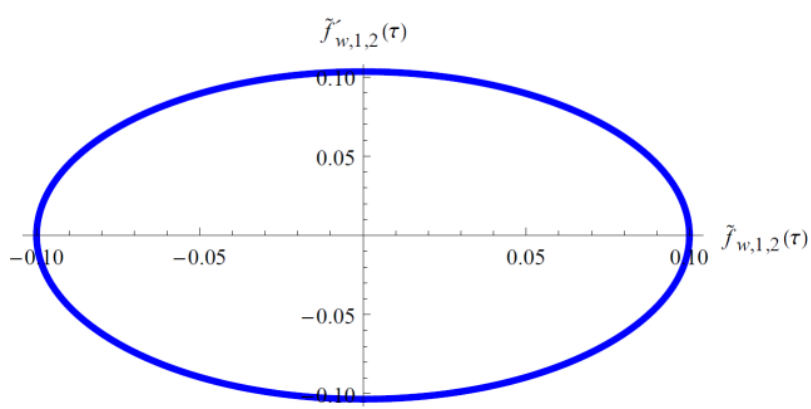

(b)

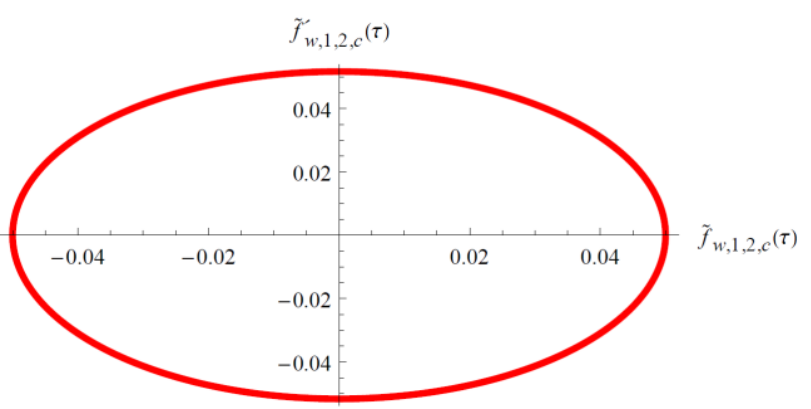

Figure 8. Phase portraits of the conjugate modes $(1,2)(\mathrm{a})$ and $(1,2, c)$ (b). Free-free SWCNT of Table 1. Modal expansion (34). Initial conditions (35). - Mode $(1,2) .-$ Mode $(1,2, c)$. Time period $\tau_{(1,2)}=6.07905$.

\subsubsection{Total energy distribution}

The evolution in time of the total energy distribution $\tilde{E}(\eta, \tau)$ of the conjugate modes $(1,2),(1,2, c)$ in the linear field along the SWCNT axis, by assuming the circumferential coordinate $\theta=0$, is shown in Figure 9. An initial energy is imposed on the edge sections along the SWCNT axis $(\eta=(0,1))$ : it can be noted that this energy is preserved in amplitude throughout the time.

(a)

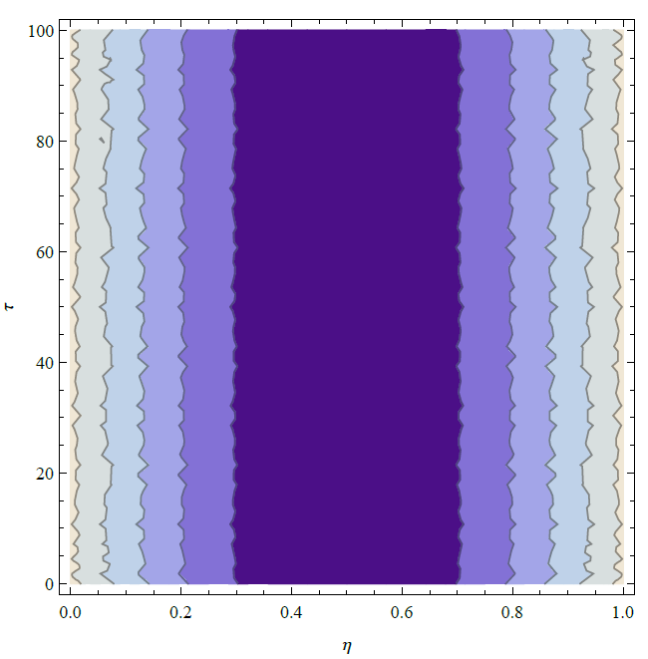

(b)

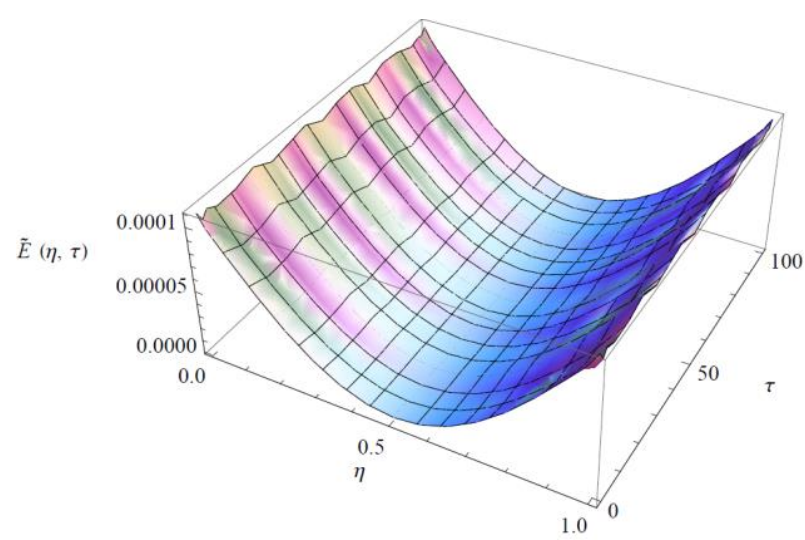

Figure 9. Contour plot (a) and 3D plot (b) of the total energy distribution $\tilde{E}(\eta, \tau)$ of the conjugate CFMs $(1,2),(1,2, c)$ along the SWCNT axis in time. Circumferential coordinate $\theta=0$. Free-free SWCNT of Table 1.

The total energy distribution $\tilde{E}(\eta, \theta, \tau)$ of the conjugate modes $(1,2),(1,2, \mathrm{c})$ over the CNT surface $(\eta, \theta)$ for different time values throughout the time period $\tau_{(1,2)}$ is shown in Figures 10-11. From these figures, it can be observed that the total energy distribution over the carbon nanotube surface is periodic along the circumferential direction $\theta$ and symmetric along the longitudinal direction $\eta$. 
$\tau=0$

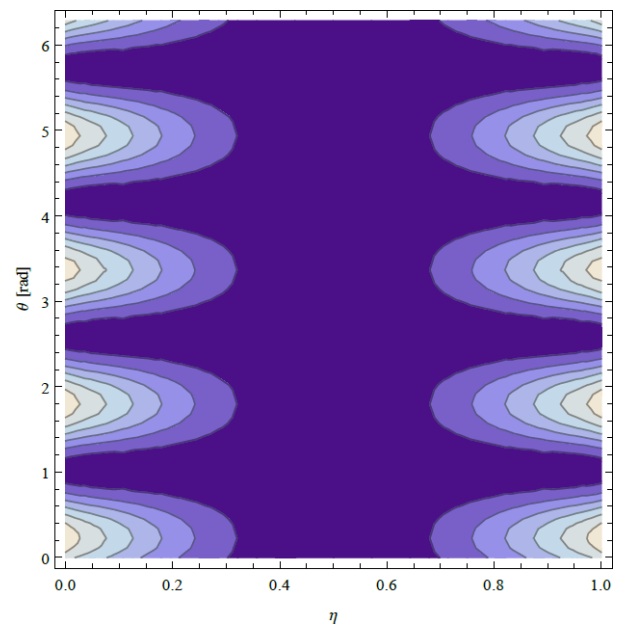

$\tau=2 \times \tau_{(1,2)} / 11$

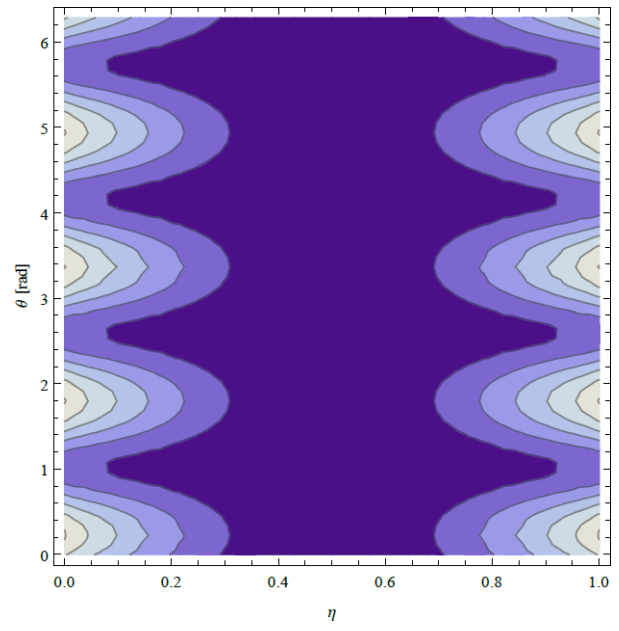

$\tau=4 \times \tau_{(1,2)} / 11$

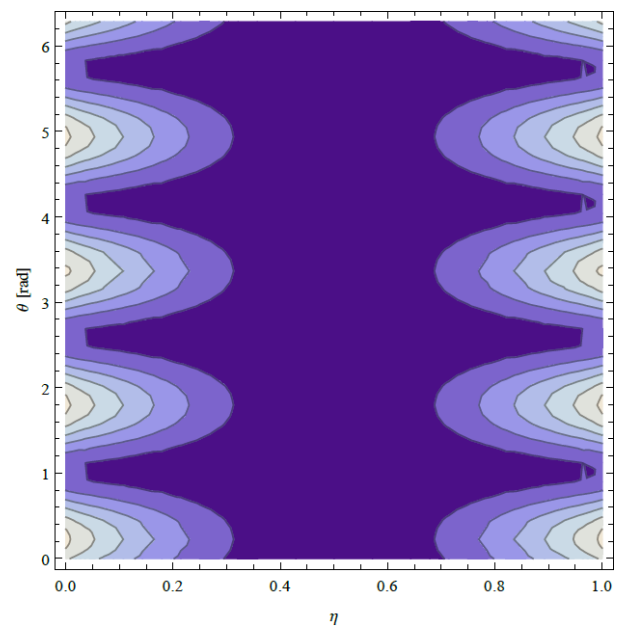

$\tau=\tau_{(1,2)} / 11$

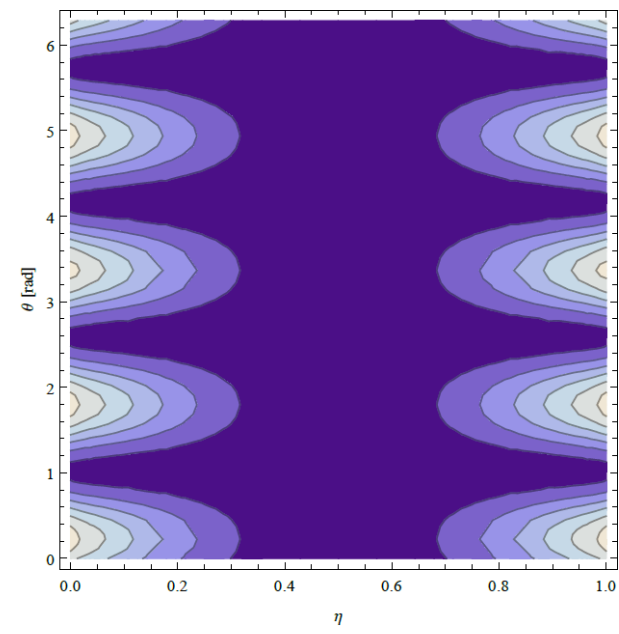

$\tau=3 \times \tau_{(1,2)} / 11$

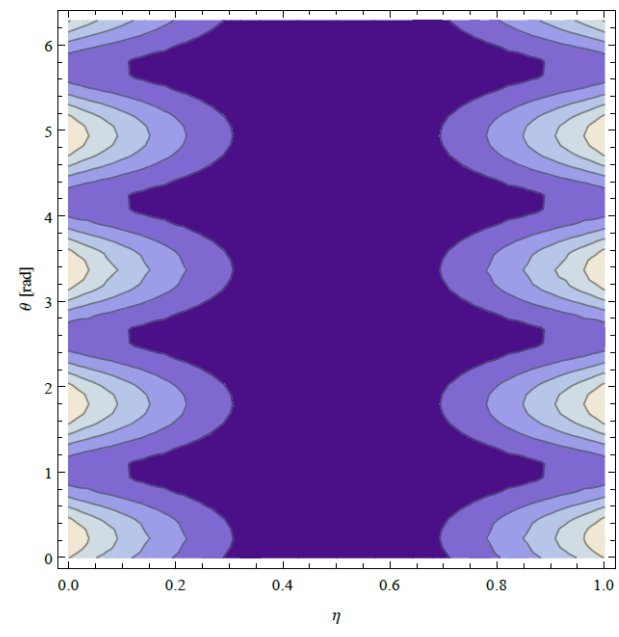

$\tau=5 \times \tau_{(1,2)} / 11$

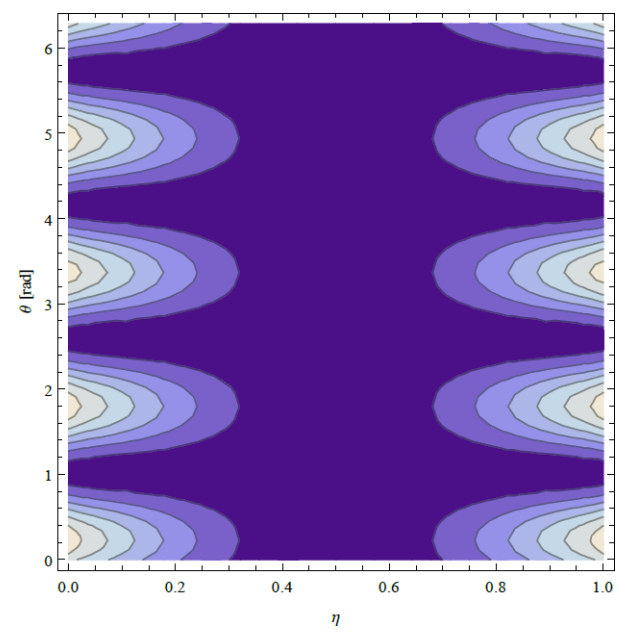


$\tau=6 \times \tau_{(1,2)} / 11$

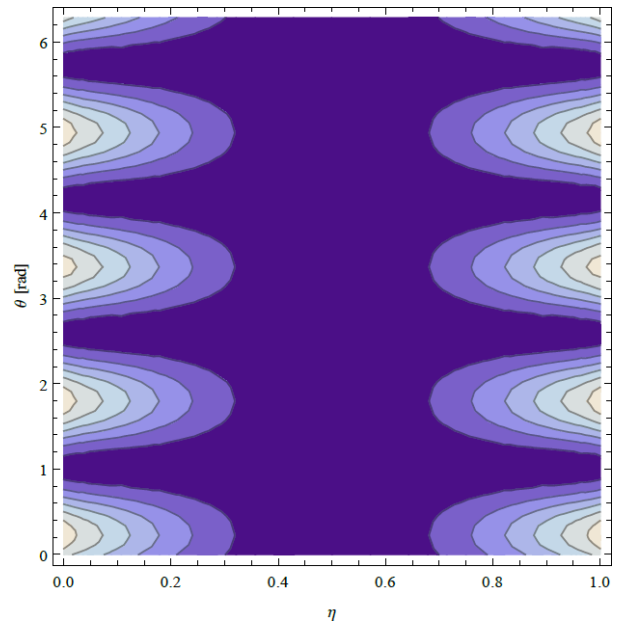

$\tau=8 \times \tau_{(1,2)} / 11$

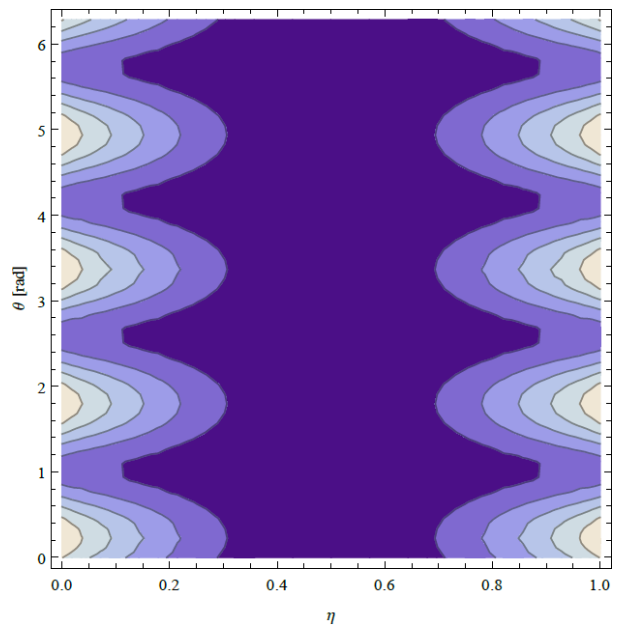

$\tau=10 \times \tau_{(1,2)} / 11$

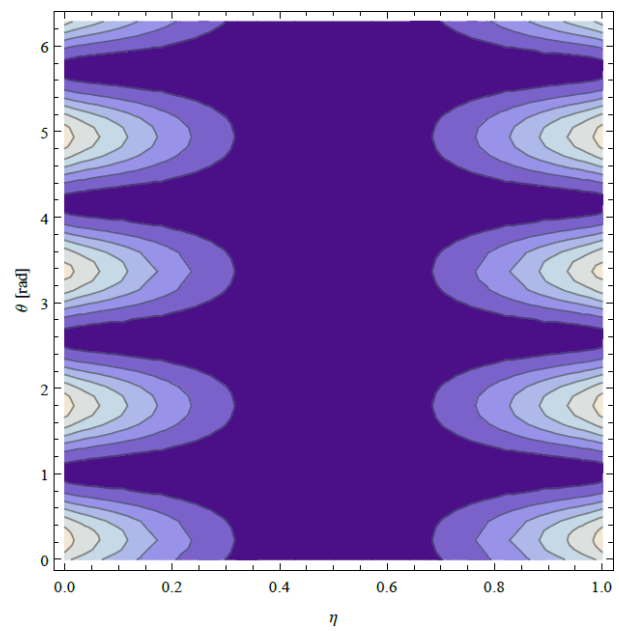

$\tau=7 \times \tau_{(1,2)} / 11$

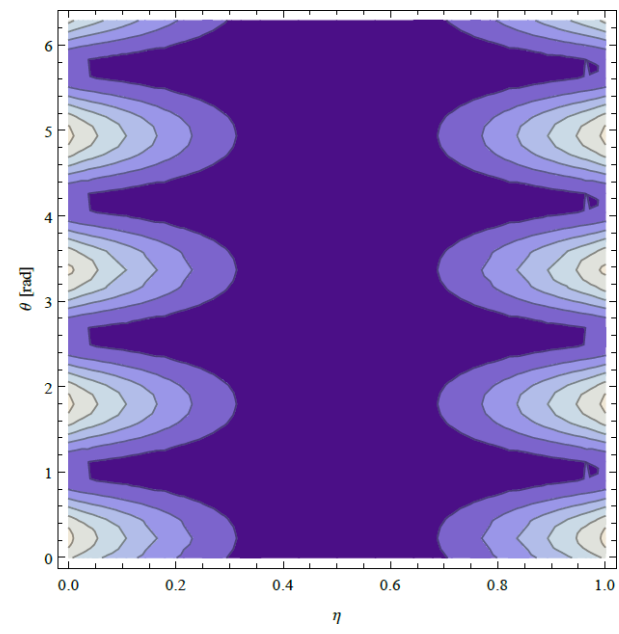

$\tau=9 \times \tau_{(1,2)} / 11$

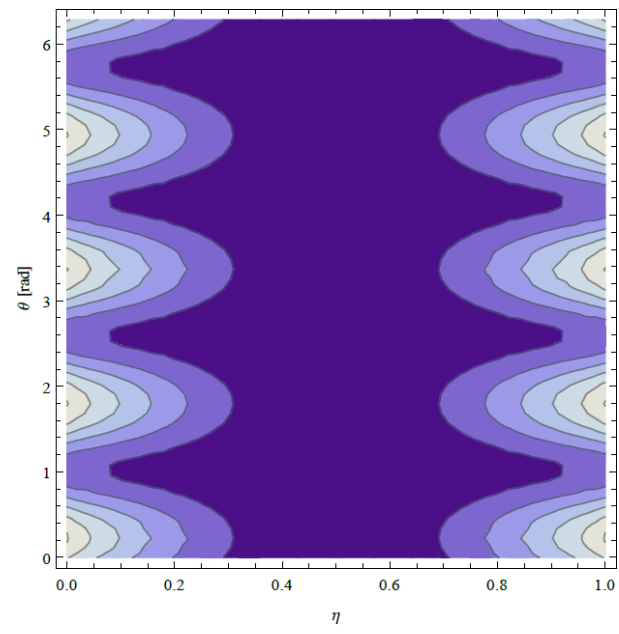

$\tau=\tau_{(1,2)}$

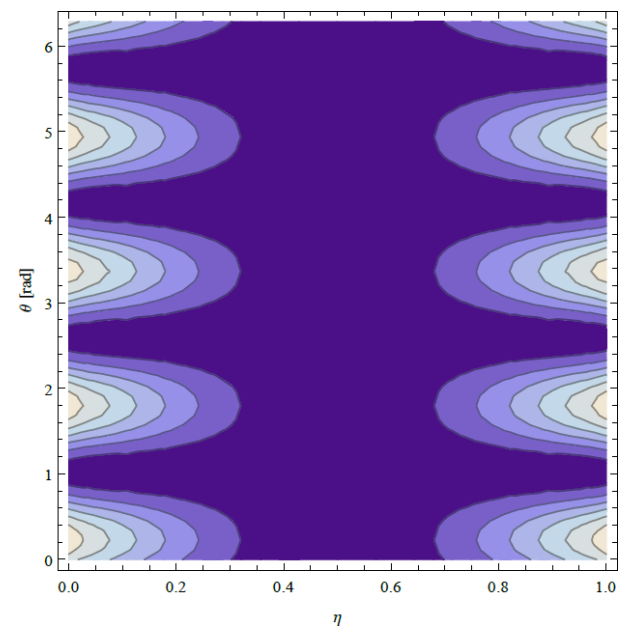

Figure 10. Contour plot of the total energy distribution $\tilde{E}(\eta, \theta, \tau)$ of the conjugate modes $(1,2),(1,2, c)$ over the SWCNT surface $(\eta, \theta)$ for different time values throughout the reference period $\tau_{(1,2)}$. 
Nonlinear resonance interaction between conjugate circumferential flexural modes in single-walled carbon nanotubes

$$
\tau=0
$$

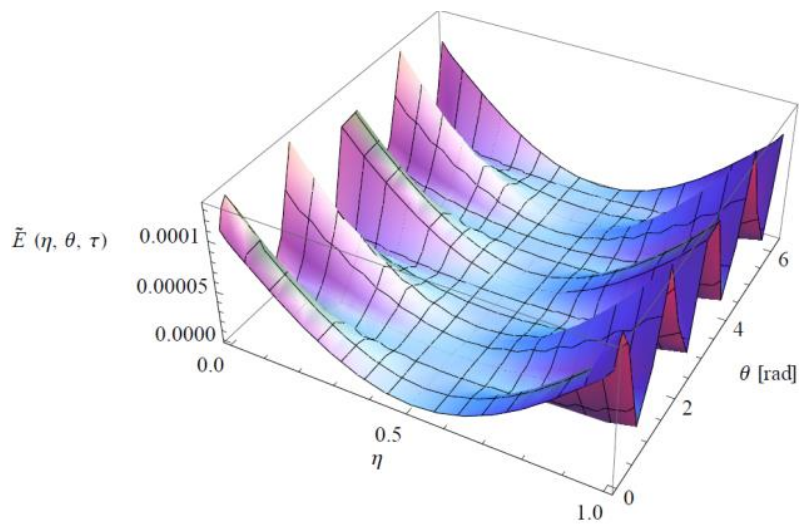

$\tau=2 \times \tau_{(1,2)} / 11$

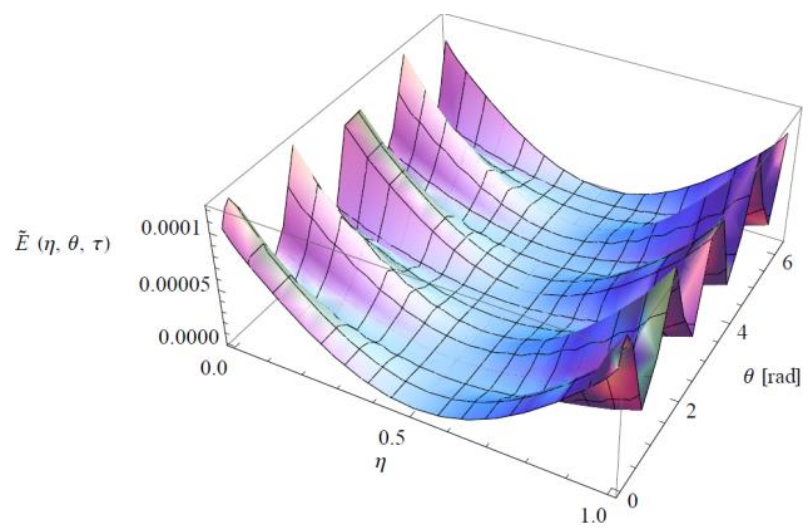

$\tau=4 \times \tau_{(1,2)} / 11$

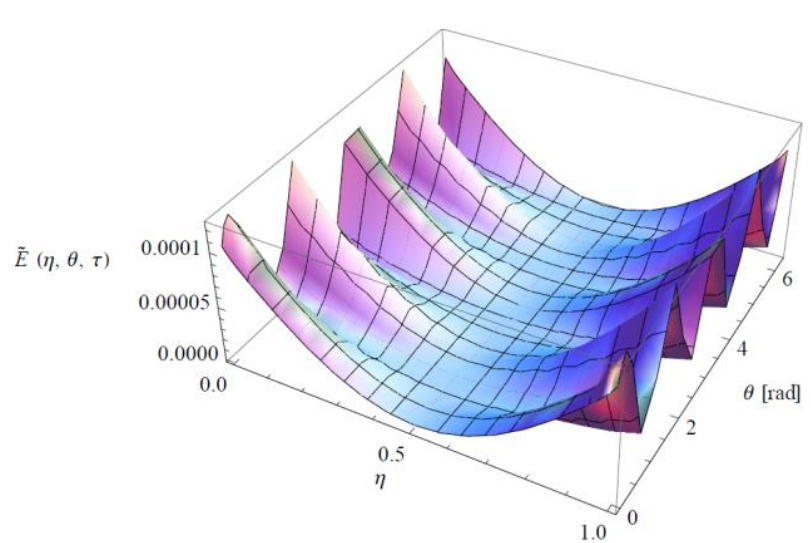

$\tau=\tau_{(1,2)} / 11$

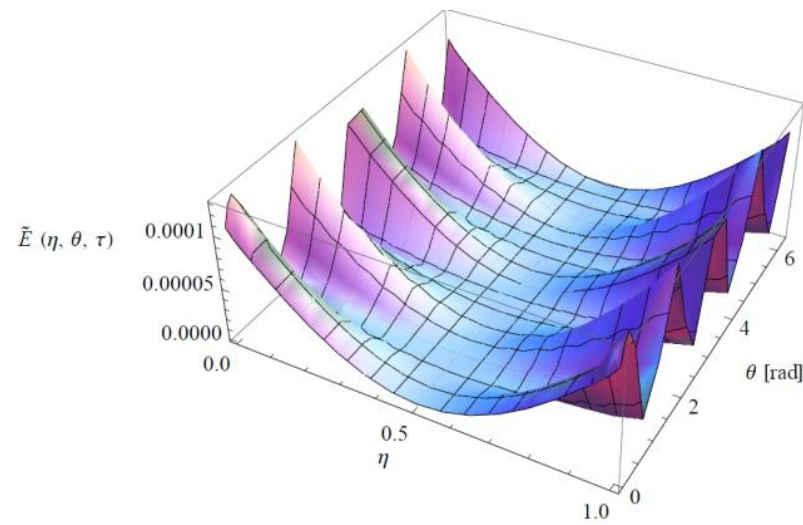

$\tau=3 \times \tau_{(1,2)} / 11$

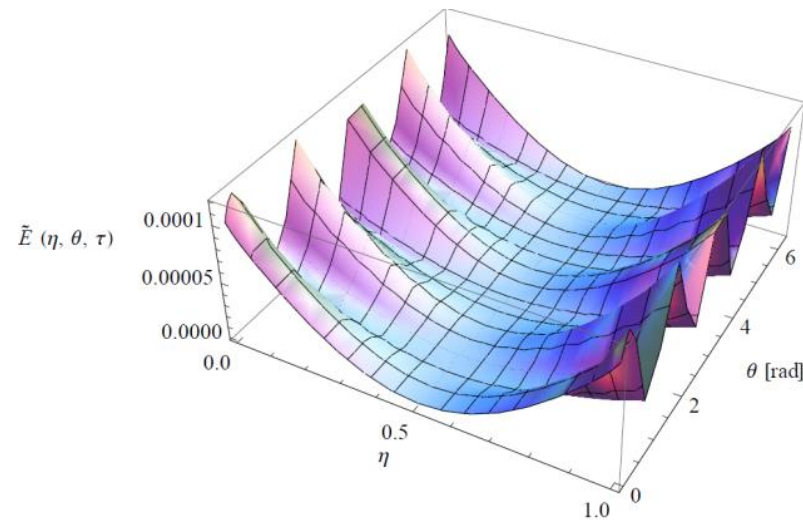

$\tau=5 \times \tau_{(1,2)} / 11$

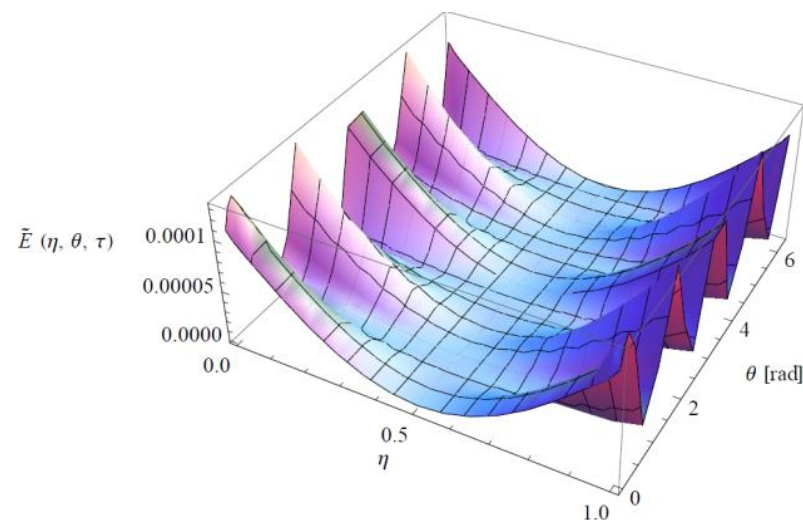


Nonlinear resonance interaction between conjugate circumferential flexural modes in single-walled carbon nanotubes

$\tau=6 \times \tau_{(1,2)} / 11$

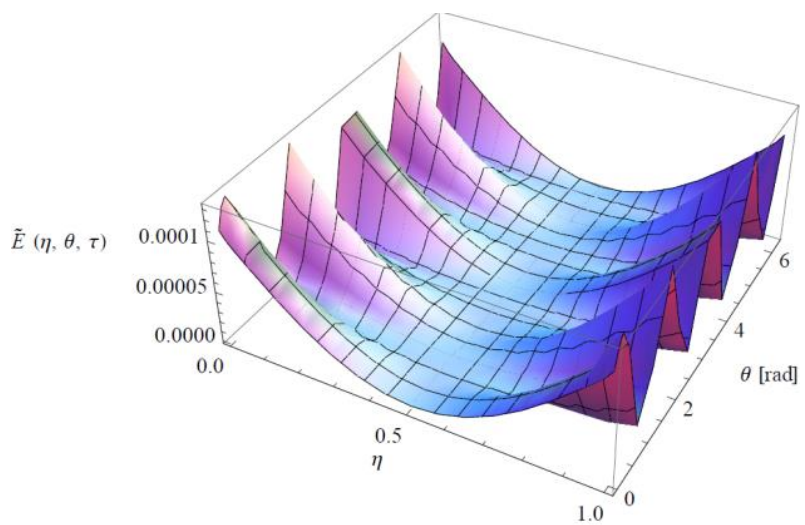

$\tau=8 \times \tau_{(1,2)} / 11$

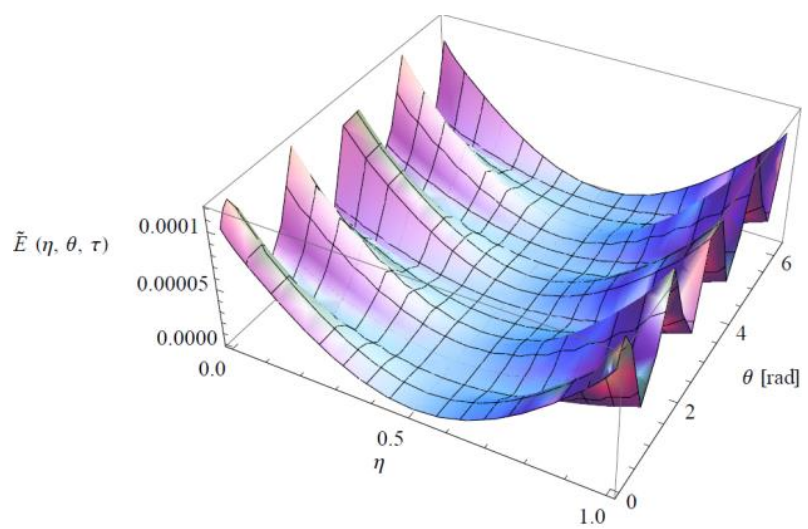

$\tau=10 \times \tau_{(1,2)} / 11$

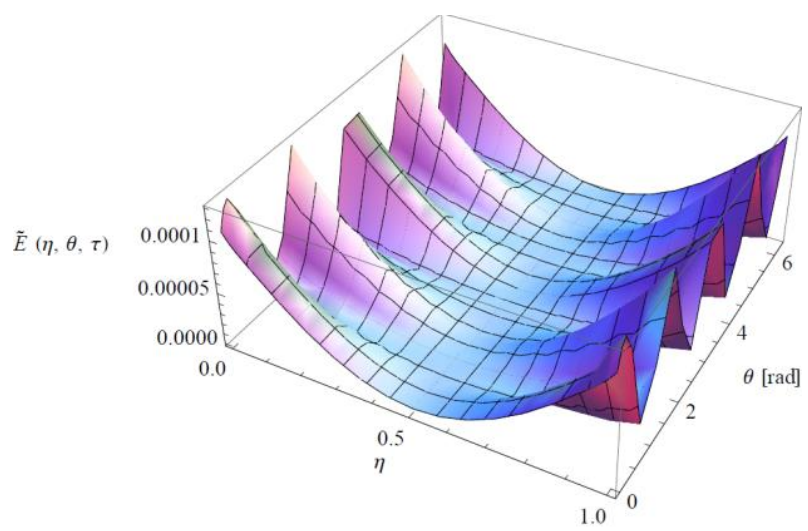

$\tau=7 \times \tau_{(1,2)} / 11$

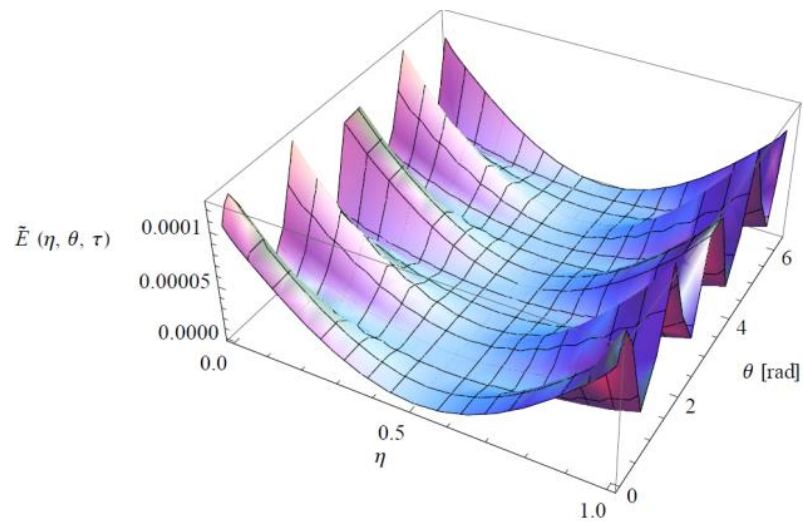

$\tau=9 \times \tau_{(1,2)} / 11$

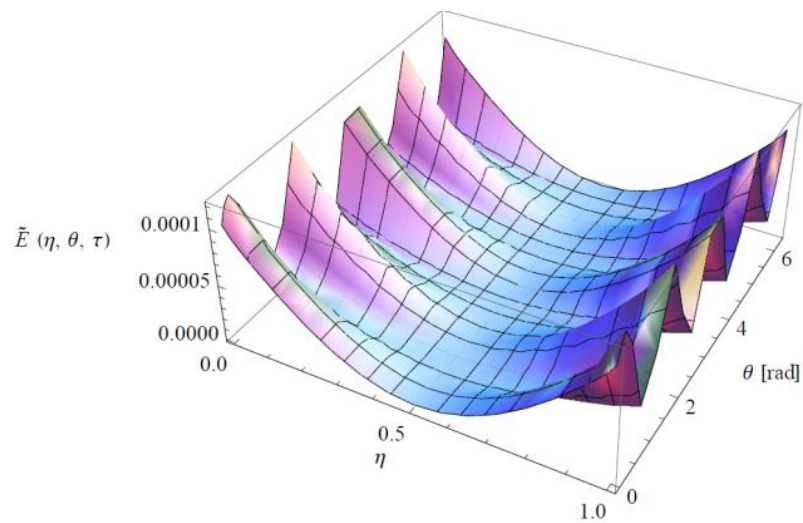

$\tau=\tau_{(1,2)}$

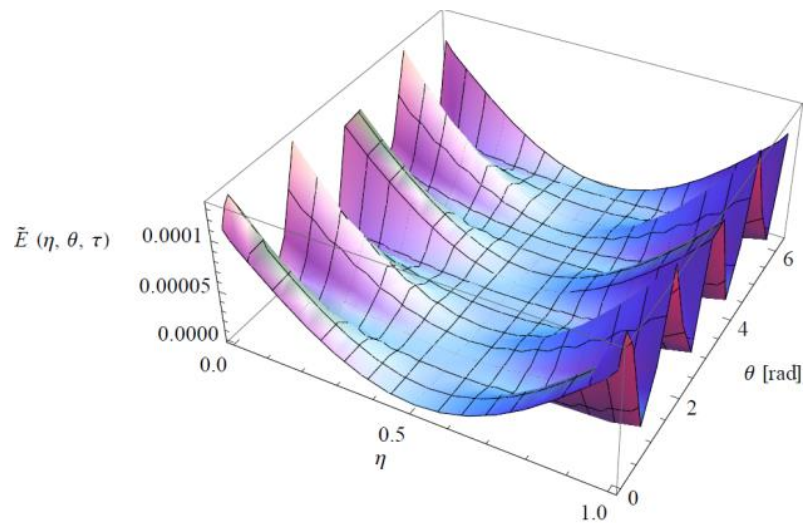

Figure 11. 3D plot of the total energy distribution $\tilde{E}(\eta, \theta, \tau)$ of the conjugate modes $(1,2),(1,2, c)$ over the SWCNT surface $(\eta, \theta)$ for different time values throughout the reference period $\tau_{(1,2)}$. 


\subsubsection{Total energy conservation}

After integration along the longitudinal $\eta$ and circumferential $\theta$ coordinates, the evolution in time of the elastic strain and kinetic energy of the conjugate CFMs $(1,2),(1,2, c)$ is found, see Figure 12(a). The evolution in time of the total energy is shown in Figure 12(b): since no damping is included in this model, then the dynamical system is conservative over the time.

(a)

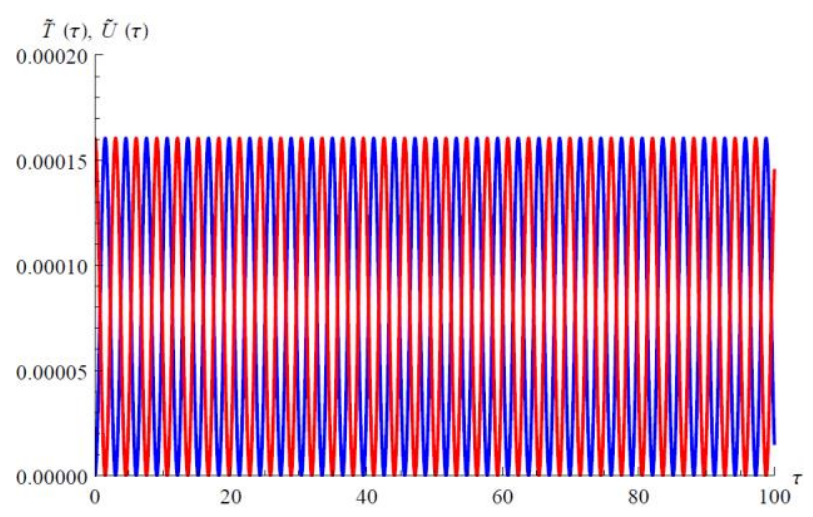

(b)

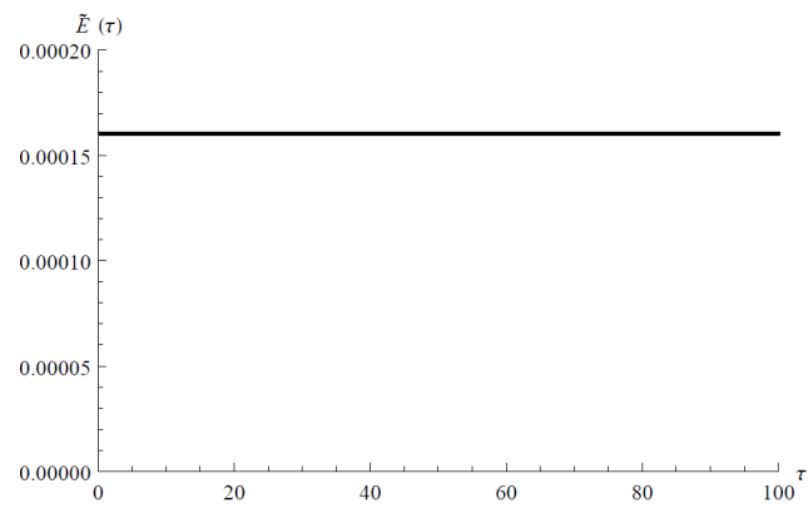

Figure 12. Evolution in time of the energy of the conjugate modes $(1,2),(1,2, c)$. — Elastic strain energy.

- Kinetic energy. (a) Comparison of the two energies. (d) Total energy conservation.

In the following, the previous analyses carried out for the conjugate CFMs $(1,2),(1,2, c)$ in the linear field will be extended to the nonlinear field: nonlinear time histories, frequency spectra and phase portraits will be found. Moreover, the nonlinear total energy distribution over the CNT surface in the time will be obtained. The aim of these analyses is the investigation of the phenomena arising in presence of nonlinear resonance interaction and energy exchange between the two conjugate CFMs. 


\subsection{Nonlinear analysis}

In the nonlinear vibration analysis, the full expression of the dimensionless elastic strain energy (9), containing terms up to the fourth order (cubic nonlinearity), is considered.

\subsubsection{Convergence analysis}

The first step of the nonlinear study is the convergence analysis in terms of the modal expansion (27-29): it is carried out by selecting the vibration modes to be inserted into the modal expansion providing an accurate description of the actual nonlinear behaviour of the SWCNT.

In particular, the aim of this convergence analysis is to find a modal expansion with the minimum number of degrees of freedom that allows the nonlinear behaviour of the sum of the time histories of the two conjugate modes $(1,2),(1,2, c)$ to be correctly described.

An initial two-mode approximation involving only the conjugate modes $(1,2)$ and $(1,2, c)$ is applied (6 dof model); the convergence is then checked by adding suitable modes to the conjugate ones, i.e., asymmetric and axisymmetric modes, in the modal expansion (27-29), as shown in Table 5.

The modal initial conditions $\tilde{f}_{u, j, n}(0), \tilde{f}_{v, j, n}(0), \tilde{f}_{w, j, n}(0)$ applied in this convergence analysis are

$$
\begin{array}{clc}
\tilde{f}_{u, 1,2}(0)=0.1 & \tilde{f}_{v, 1,2}(0)=0.1 & \tilde{f}_{w, 1,2}(0)=0.1 \\
\tilde{f}_{u, 1,2, c}(0)=0.05 & \tilde{f}_{v, 1,2, c}(0)=0.05 & \tilde{f}_{w, 1,2, c}(0)=0.05 \\
\tilde{f}_{u, j, n}(0)=0.001 & \tilde{f}_{v, j, n}(0)=0.001 & \tilde{f}_{w, j, n}(0)=0.001
\end{array}
$$

\begin{tabular}{|c|c|c|c|c|c|c|c|c|c|}
\hline$(j, n)$ & $(1,2)$ & $(1,2, c)$ & $(1,4)$ & $(1,4, c)$ & $(3,2)$ & $(3,2, c)$ & $(1,0)$ & $(3,0)$ & ERROR $_{\mathrm{RMS}} \%$ \\
\hline 6 dof model & $u, v, w$ & $u, v, w$ & - & - & - & - & - & - & 12.8 \\
\hline 8 dof model & $u, v, w$ & $u, v, w$ & - & - & - & - & $u, w$ & - & 8.63 \\
\hline 14 dof model & $u, v, w$ & $u, v, w$ & $u, v, w$ & $u, v, w$ & - & - & $u, w$ & - & 4.54 \\
\hline 20 dof model & $u, v, w$ & $u, v, w$ & $u, v, w$ & $u, v, w$ & $u, v, w$ & $u, v, w$ & $u, w$ & - & 2.23 \\
\hline 22 dof model & $u, v, w$ & $u, v, w$ & $u, v, w$ & $u, v, w$ & $u, v, w$ & $u, v, w$ & $u, w$ & $u, w$ & \\
\hline
\end{tabular}

Table 5. Nonlinear convergence analysis of the sum of the time histories of the two conjugate modes $(1,2),(1,2, c)$.

Nonlinear modal expansion (27-29). Modal initial conditions (36). 22 dof reference model. ERROR $\mathrm{RMS}_{\%}$ in the time domain for the 6 dof model, 8 dof model, 14 dof model and 20 dof model. Free-free SWCNT of Table 1. 
The convergence is reached by means of a 22 dof model, described in Table 5, which is assumed as a reference model; the percentage root mean square error in the time domain $\left(\mathrm{ERROR}_{\mathrm{RMS}} \%\right)$ with respect to the reference model is used as a comparison parameter for the other nonlinear models.

In Figure 13, the nonlinear convergence analysis of the sum of the time histories of the conjugate modes $(1,2),(1,2, c)$ obtained applying the nonlinear modal expansion (27-29) with the modal initial conditions (36) for the different nonlinear models of Table 5 is shown. The 22 dof nonlinear model is assumed as a reference model.

From this convergence analysis, it can be found that the smallest model able to predict the nonlinear dynamics of the system with an acceptable accuracy is the 14 dof model (ERROR RMS $\left._{0}=4 \div 5\right)$. The main weakness of the 6-8 dof models is the insufficient number of asymmetric and axisymmetric modes, which are very important for properly modelling bending deformation (asymmetric modes) and circumferential stretching (axisymmetric modes) during the modal vibration, see Refs. [52,53]. Since the 14 dof model provides accurate results with the minimal computational effort, then in the following this model will be used for studying the nonlinear oscillations of the two conjugate modes $(1,2),(1,2, c)$. By inserting into modal expansion (27-29)

- $\operatorname{modes}(1,2),(1,2, c),(1,4),(1,4, c),(1,0)$ along the longitudinal displacement $u$

- modes $(1,2),(1,2, c),(1,4),(1,4, c)$ along the circumferential displacement $v$

- $\operatorname{modes}(1,2),(1,2, c),(1,4),(1,4, c),(1,0)$ along the radial displacement $w$

the resulting nonlinear modal expansion is written in the form

$$
\begin{aligned}
\tilde{u}(\eta, \theta, \tau)= & \tilde{U}^{(1,2)}(\eta, \theta) \tilde{f}_{u, 1,2}(\tau)+\tilde{U}^{(1,2, c)}(\eta, \theta) \tilde{f}_{u, 1,2, c}(\tau)+\tilde{U}^{(1,4)}(\eta, \theta) \tilde{f}_{u, 1,4}(\tau)+ \\
+ & \tilde{U}^{(1,4, c)}(\eta, \theta) \tilde{f}_{u, 1,4, c}(\tau)+\tilde{U}^{(1,0)}(\eta, \theta) \tilde{f}_{u, 1,0}(\tau) \\
& \tilde{v}(\eta, \theta, \tau)=\tilde{V}^{(1,2)}(\eta, \theta) \tilde{f}_{v, 1,2}(\tau)+\tilde{V}^{(1,2, c)}(\eta, \theta) \tilde{f}_{v, 1,2, c}(\tau)+ \\
& +\tilde{V}^{(1,4)}(\eta, \theta) \tilde{f}_{v, 1,4}(\tau)+\tilde{V}^{(1,4, c)}(\eta, \theta) \tilde{f}_{v, 1,4, c}(\tau) \\
\tilde{w}(\eta, \theta, \tau)= & \tilde{W}^{(1,2)}(\eta, \theta) \tilde{f}_{w, 1,2}(\tau)+\tilde{W}^{(1,2, c)}(\eta, \theta) \tilde{f}_{w, 1,2, c}(\tau)+\tilde{W}^{(1,4)}(\eta, \theta) \tilde{f}_{w, 1,4}(\tau)+ \\
+ & \tilde{W}^{(1,4, c)}(\eta, \theta) \tilde{f}_{w, 1,4, c}(\tau)+\tilde{W}^{(1,0)}(\eta, \theta) \tilde{f}_{w, 1,0}(\tau)
\end{aligned}
$$

where the longitudinal and radial displacements have five terms, the circumferential displacement has four terms and the total number of degrees of freedom of the model is equal to $\left(N_{\max }=14\right)$. 
6 dof model vs. 22 dof model

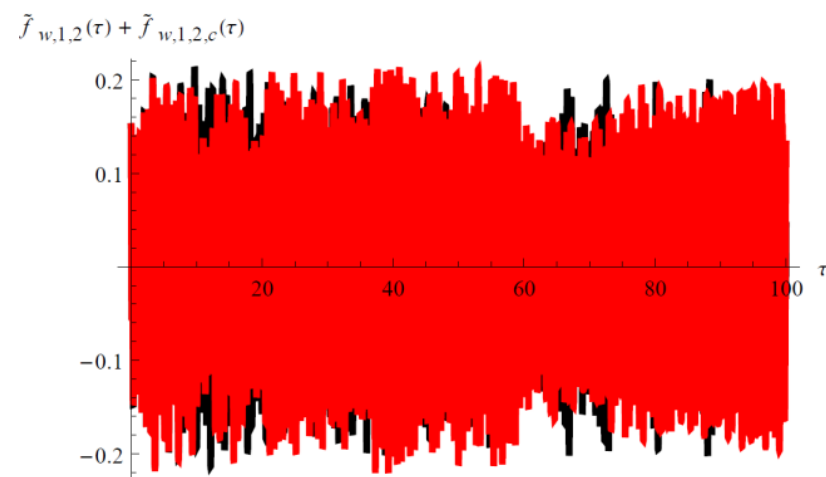

14 dof model vs. 22 dof model

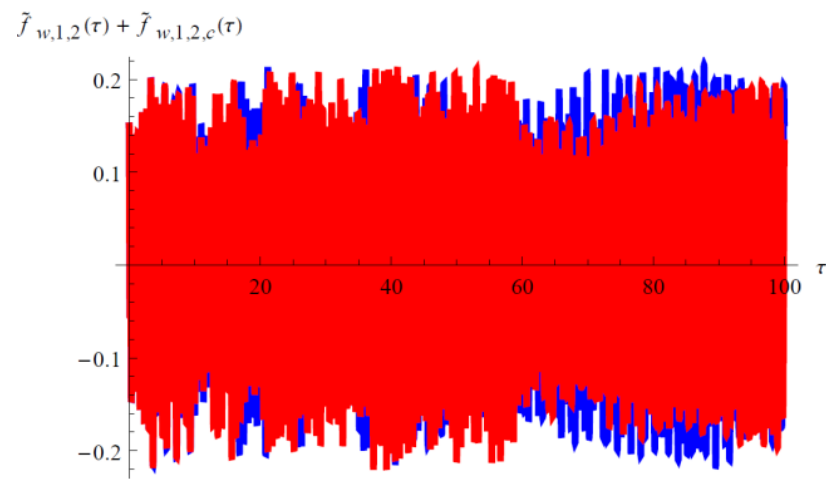

Comparisons

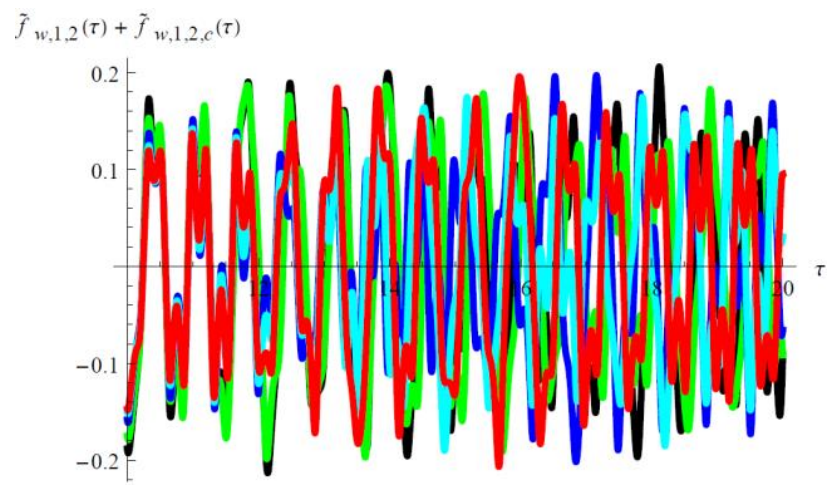

8 dof model vs. 22 dof model

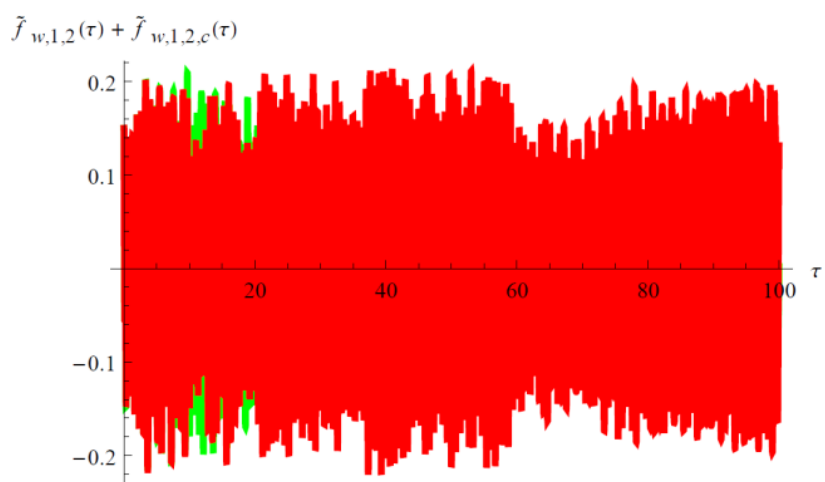

20 dof model vs 22 dof model

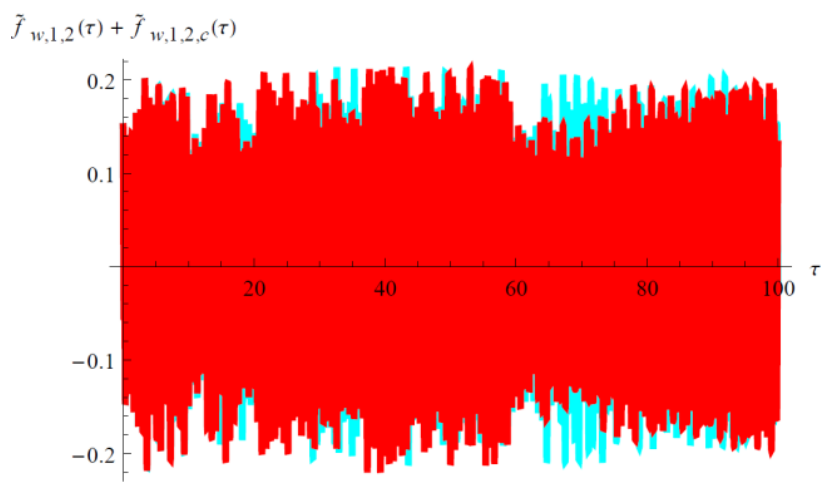

Comparisons

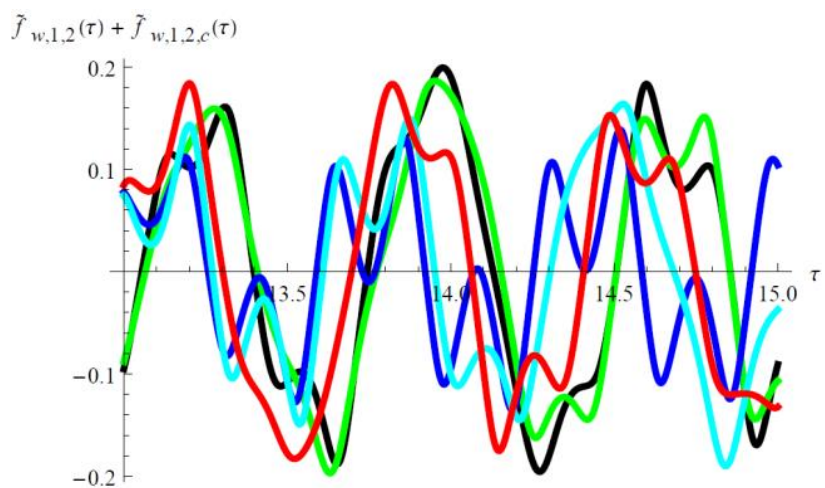

Figure 13. Nonlinear convergence analysis of the sum of the time histories of the two conjugate modes $(1,2),(1,2, c)$. Nonlinear modal expansion (27-29). Modal initial conditions (36). Nonlinear models of Table 5. 22 dof reference model. “-”, 6 dof model. “_”, 8 dof model. “-”, 14 dof model. “_,", 20 dof model. “-”, 22 dof model. 


\subsubsection{Modal initial conditions}

In this section, the minimum value of the modal initial conditions (36) which activates the nonlinear response of the conjugate mode $(1,2, c)$ is investigated. The nonlinear modal expansion (37) is used (14 dof model). The initial energy on the modes $(1,2),(1,4),(1,4, c),(1,0)$ is fixed. The value of the initial energy imposed on the mode $(1,2, c)$ is increased until its nonlinear activation, see Table 6.

In Figure 14, the time histories of the conjugate modes $(1,2),(1,2, c)$ are shown. In Figures 14(a-b-c) (cases A, B, C of Table 6) the mode $(1,2, c)$ is not activated. In Figures $14(\mathrm{~d}-\mathrm{e})$ (cases D, E of Table 6) a weak activation of the mode $(1,2, c)$ arises after suitable long time. In Figure 14(f) (case F of Table 6) the nonlinear response of the mode $(1,2, c)$ is strongly activated, and it vibrates with a large amplitude: this is caused by an energy transfer probably due to a nonlinear resonance interaction between the two conjugate CFMs.

From Table 6 and Figure 14, it is obtained that the modal initial conditions activating the nonlinear response of the conjugate mode $(1,2, c)$ are given by (Case F)

$$
\begin{array}{ccc}
\tilde{f}_{u, 1,2}(0)=0.1 & \tilde{f}_{v, 1,2}(0)=0.1 & \tilde{f}_{w, 1,2}(0)=0.1 \\
\tilde{f}_{u, 1,2, c}(0)=0.005 & \tilde{f}_{v, 1,2, c}(0)=0.005 & \tilde{f}_{w, 1,2, c}(0)=0.005 \\
\tilde{f}_{u, j, n}(0)=0.001 & \tilde{f}_{v, j, n}(0)=0.001 & \tilde{f}_{w, j, n}(0)=0.001
\end{array}
$$

\begin{tabular}{|c|c|c|c|}
\hline Case & \multicolumn{2}{|c|}{ Modal initial conditions on the modes $(1,2),(1,2, c)$} & Modal initial condition ratio \\
\hline A & $\tilde{f}_{w, 1,2}(0)=0.1$ & $\tilde{f}_{w, 1,2, c}(0)=0$ & $\tilde{f}_{w, 1,2}(0) / \tilde{f}_{w, 1,2, c}(0)=\infty$ \\
\hline B & $\tilde{f}_{w, 1,2}(0)=0.1$ & $\tilde{f}_{w, 1,2, c}(0)=0.00005$ & $\tilde{f}_{w, 1,2}(0) / \tilde{f}_{w, 1,2, c}(0)=2000$ \\
\hline $\mathrm{C}$ & $\tilde{f}_{w, 1,2}(0)=0.1$ & $\tilde{f}_{w, 1,2, c}(0)=0.0001$ & $\tilde{f}_{w, 1,2}(0) / \tilde{f}_{w, 1,2, c}(0)=1000$ \\
\hline $\mathrm{D}$ & $\tilde{f}_{w, 1,2}(0)=0.1$ & $\tilde{f}_{w, 1,2, c}(0)=0.0005$ & $\tilde{f}_{w, 1,2}(0) / \tilde{f}_{w, 1,2, c}(0)=200$ \\
\hline $\mathrm{E}$ & $\tilde{f}_{w, 1,2}(0)=0.1$ & $\tilde{f}_{w, 1,2, c}(0)=0.001$ & $\tilde{f}_{w, 1,2}(0) / \tilde{f}_{w, 1,2, c}(0)=100$ \\
\hline $\mathrm{F}$ & $\tilde{f}_{w, 1,2}(0)=0.1$ & $\tilde{f}_{w, 1,2, c}(0)=0.005$ & $\tilde{f}_{w, 1,2}(0) / \tilde{f}_{w, 1,2, c}(0)=20$ \\
\hline
\end{tabular}

Table 6. Modal initial conditions imposed on the conjugate modes $(1,2),(1,2, c)$. 
Case A: $\tilde{f}_{w, 1,2}(0)=0.1, \tilde{f}_{w, 1,2, c}(0)=0$

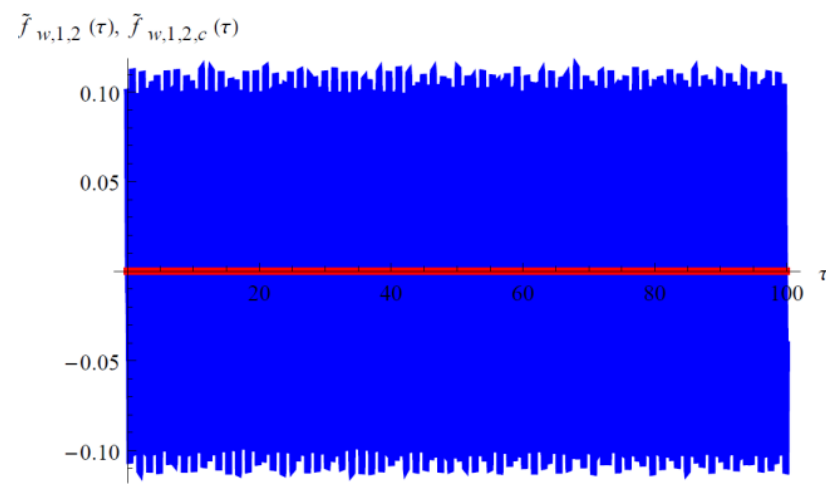

Case C: $\tilde{f}_{w, 1,2}(0)=0.1, \tilde{f}_{w, 1,2, c}(0)=0.0001$

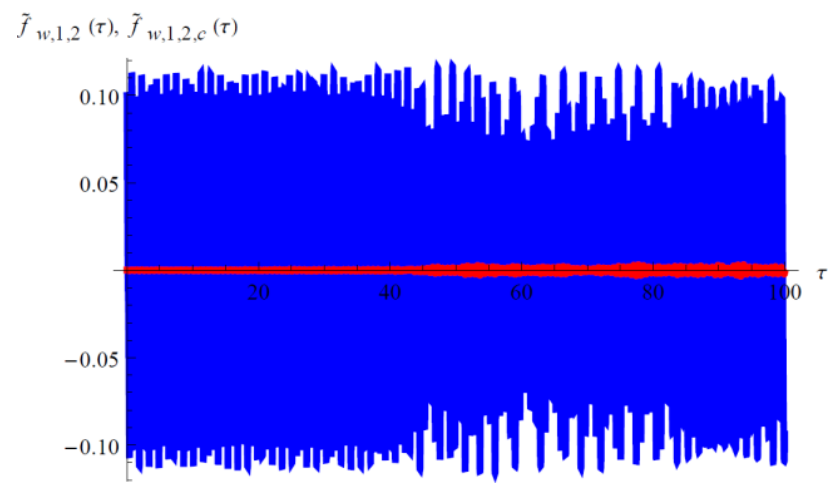

Case E: $\tilde{f}_{w, 1,2}(0)=0.1, \tilde{f}_{w, 1,2, c}(0)=0.001$

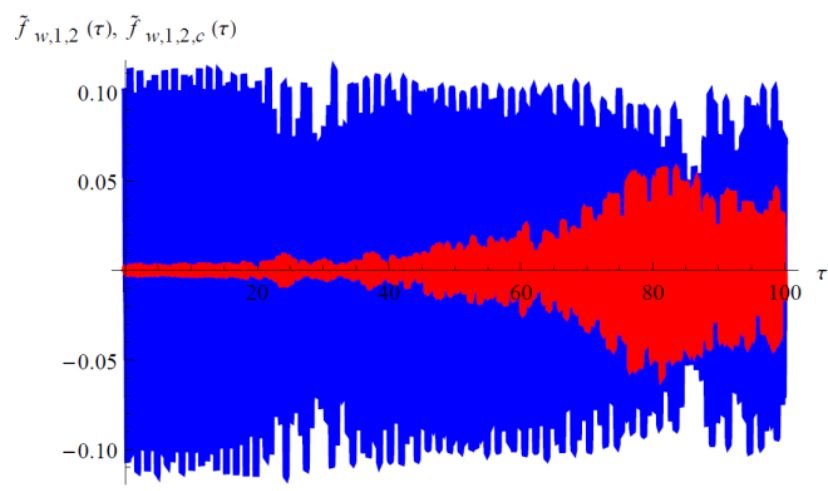

Case B: $\tilde{f}_{w, 1,2}(0)=0.1, \tilde{f}_{w, 1,2, c}(0)=0.00005$

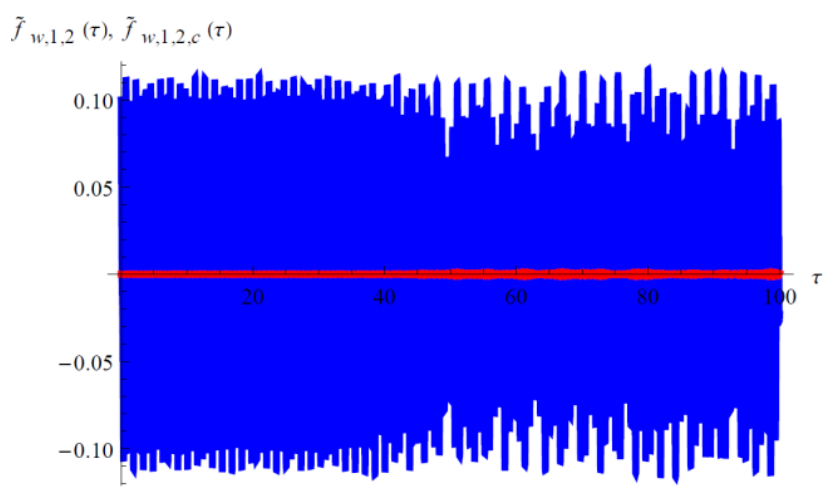

Case D: $\tilde{f}_{w, 1,2}(0)=0.1, \tilde{f}_{w, 1,2, c}(0)=0.0005$

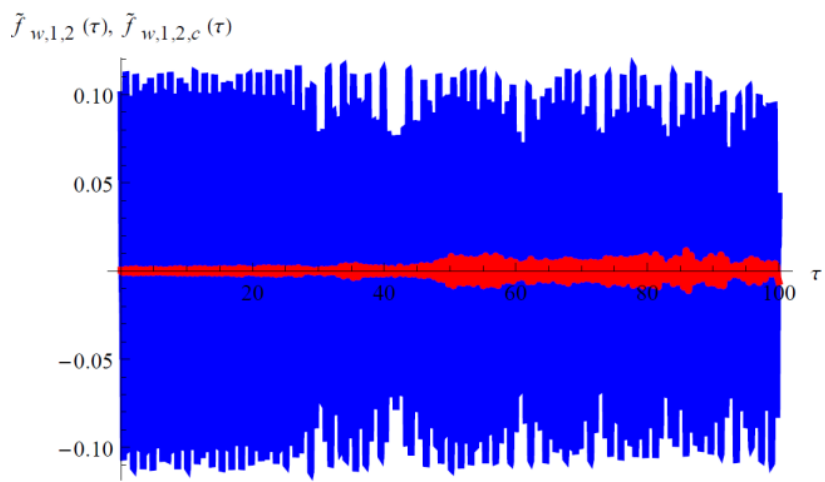

Case F: $\tilde{f}_{w, 1,2}(0)=0.1, \tilde{f}_{w, 1,2, c}(0)=0.005$

$\tilde{f}_{w, 1,2}(\tau), \tilde{f}_{w, 1,2, c}(\tau)$

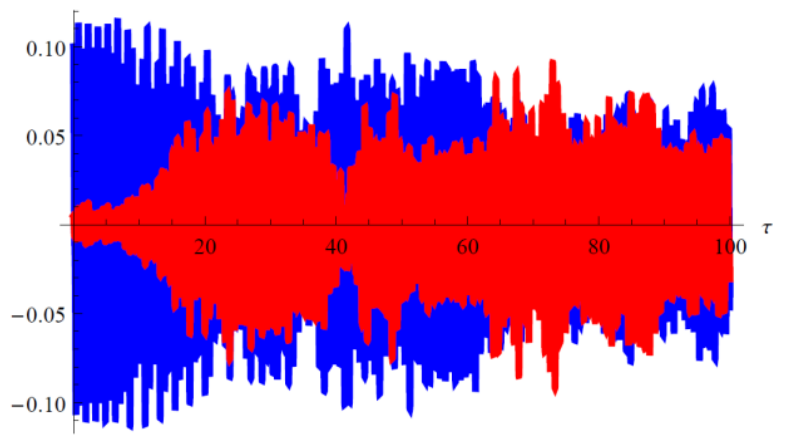

Figure 14. Nonlinear time histories of the conjugate modes $(1,2),(1,2, c)$ corresponding to the different modal initial conditions listed in Table 6. Nonlinear modal expansion (37). 14 dof model. “-”, mode $(1,2)$. “-”, mode $(1,2, c)$. 


\subsubsection{Time histories, frequency spectra, phase portraits}

The time histories of the conjugate modes $(1,2),(1,2, c)$ of the CNT are shown in Figure 15(a), the corresponding frequency spectra are presented in Figure 15(b). These plots clearly demonstrate that the vibration is a chaotic motion (no time period), i.e. the CNT is vibrating in nonlinear conditions, where the resonance interaction gives rise to strong energy exchange between the conjugate CFMs.

(a)

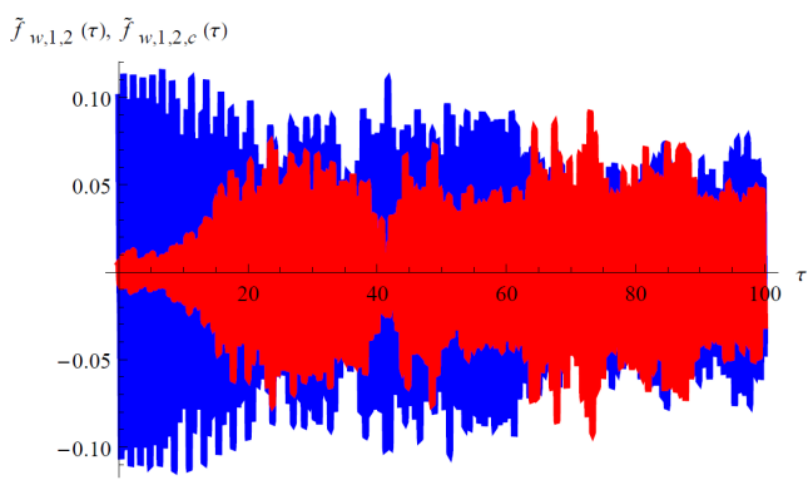

(b)

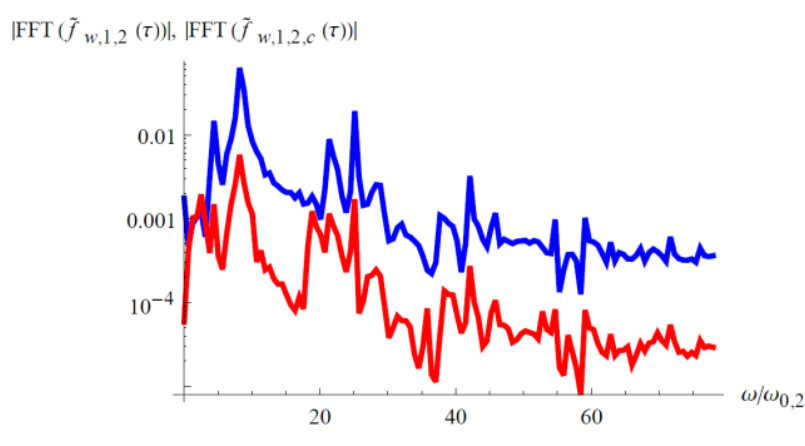

Figure 15. Time histories (a) and frequency spectra (b) of the two conjugate modes $(1,2),(1,2, c)$. Free-free SWCNT of Table 1. Nonlinear modal expansion (37). Modal initial conditions (38). - Mode (1,2). - Mode $(1,2, c)$.

Phase portraits of the mode $(1,2)$ for different times are shown in Figure 16. By calculating the first (lowest) root of the equation $\tilde{f}_{w, 1,2}(\tau)=\tilde{f}_{w, 1,2}(0)=0.1$, the time $\tau=0.77834$ is obtained, which is not a time period.

Similarly, phase portraits of the conjugate mode $(1,2, c)$ for different times are reported in Figure 17. Calculating the first (lowest) root of the equation $\tilde{f}_{w, 1,2, c}(\tau)=\tilde{f}_{w, 1,2, c}(0)=0.005$, the time $\tau=0.78436$ is found, which is different from the time value previously obtained for the mode $(1,2)$. From Figures 16-17 (phase portraits) it is confirmed the non-periodicity of the motion of the CNT in nonlinear field (i.e. chaotic motion) observed in Figure 15 (time histories and frequency spectra). 
(a)
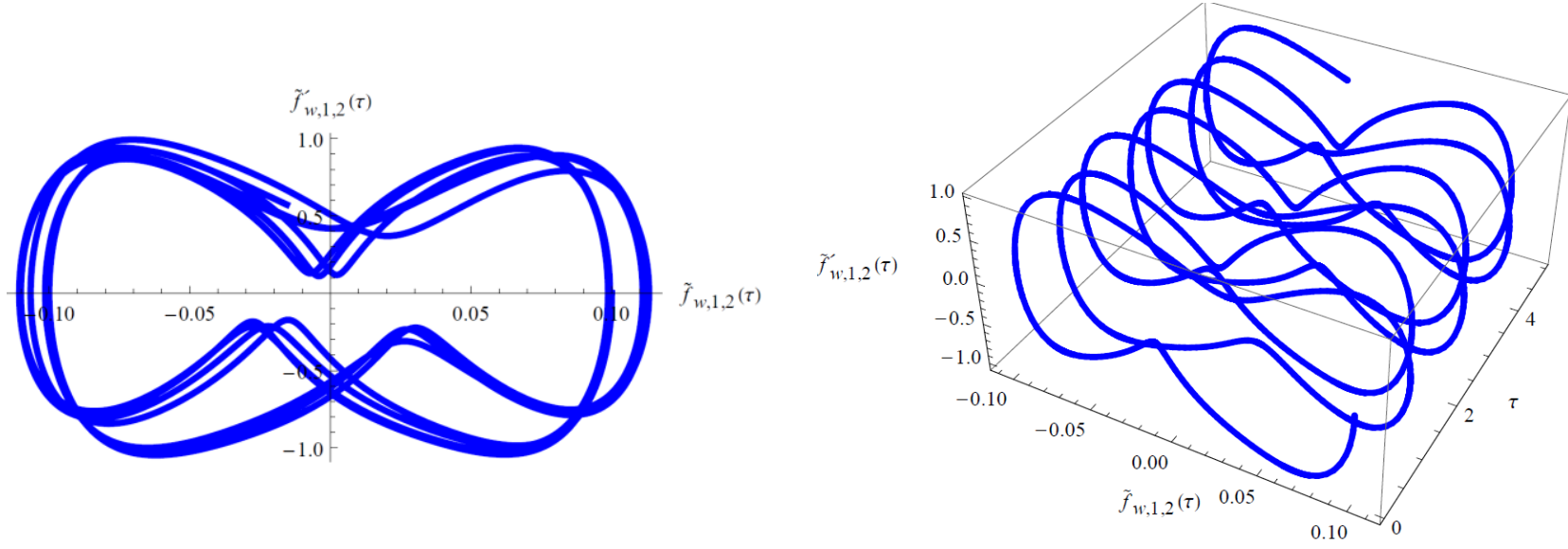

(b)
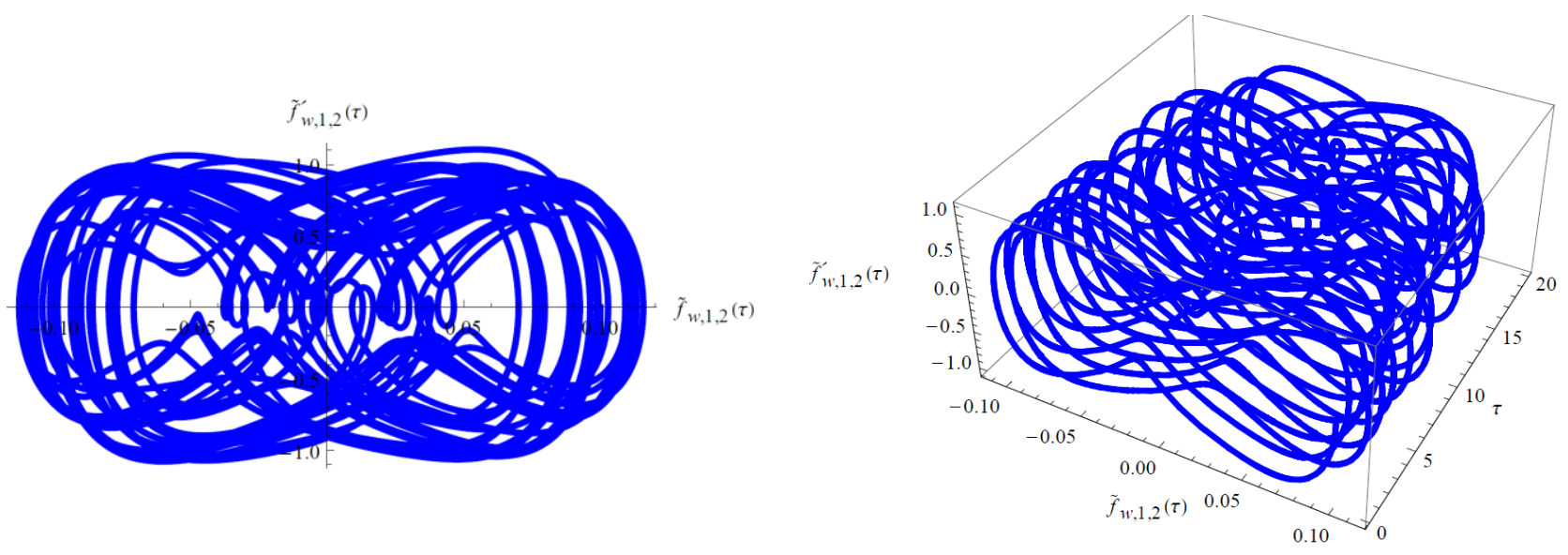

(c)
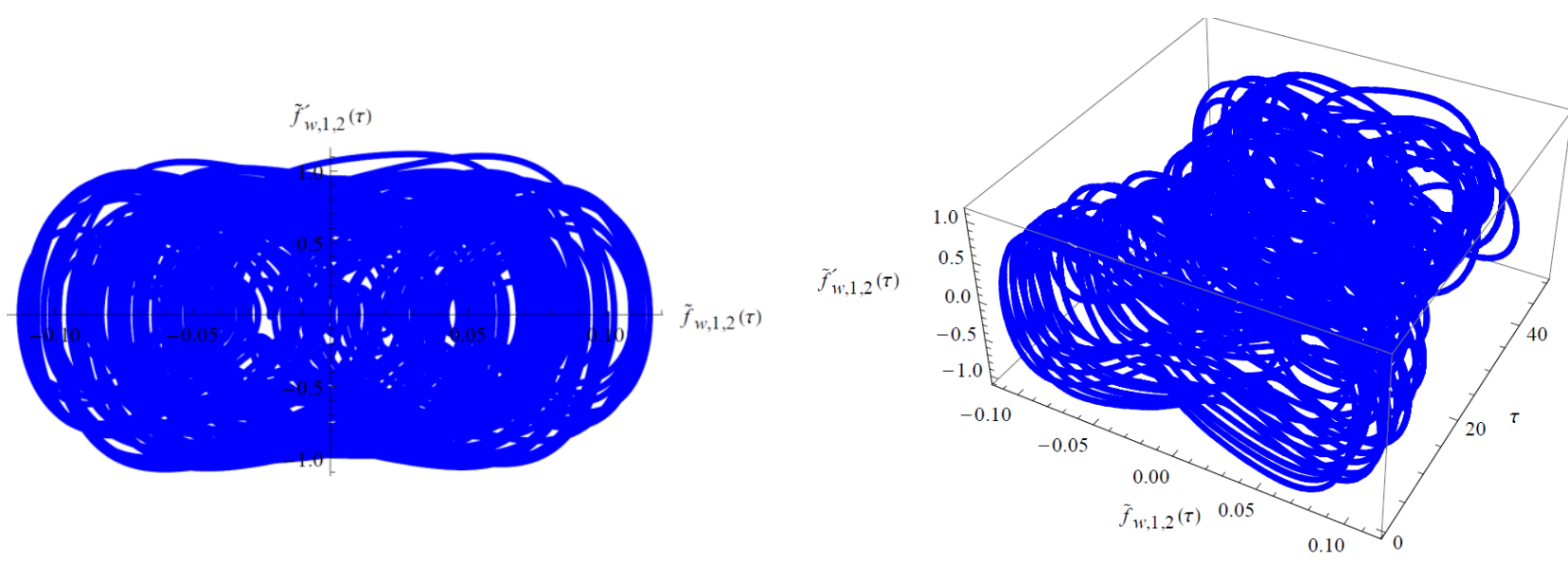

Figure 16. 2D and 3D phase portraits of the mode $(1,2)$. Free-free SWCNT of Table 1. Nonlinear modal expansion (37). Modal initial conditions (38). (a) Time $\tau=5$. (b) Time $\tau=20$. (c) Time $\tau=50$. 
(a)
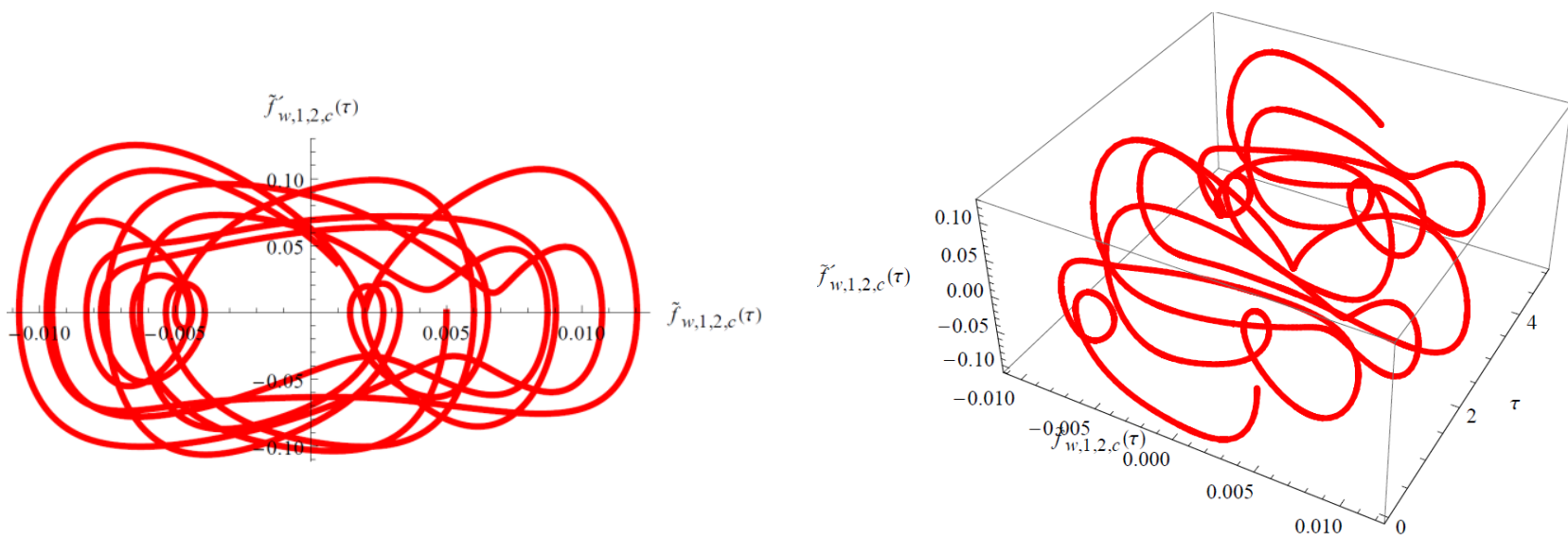

(b)
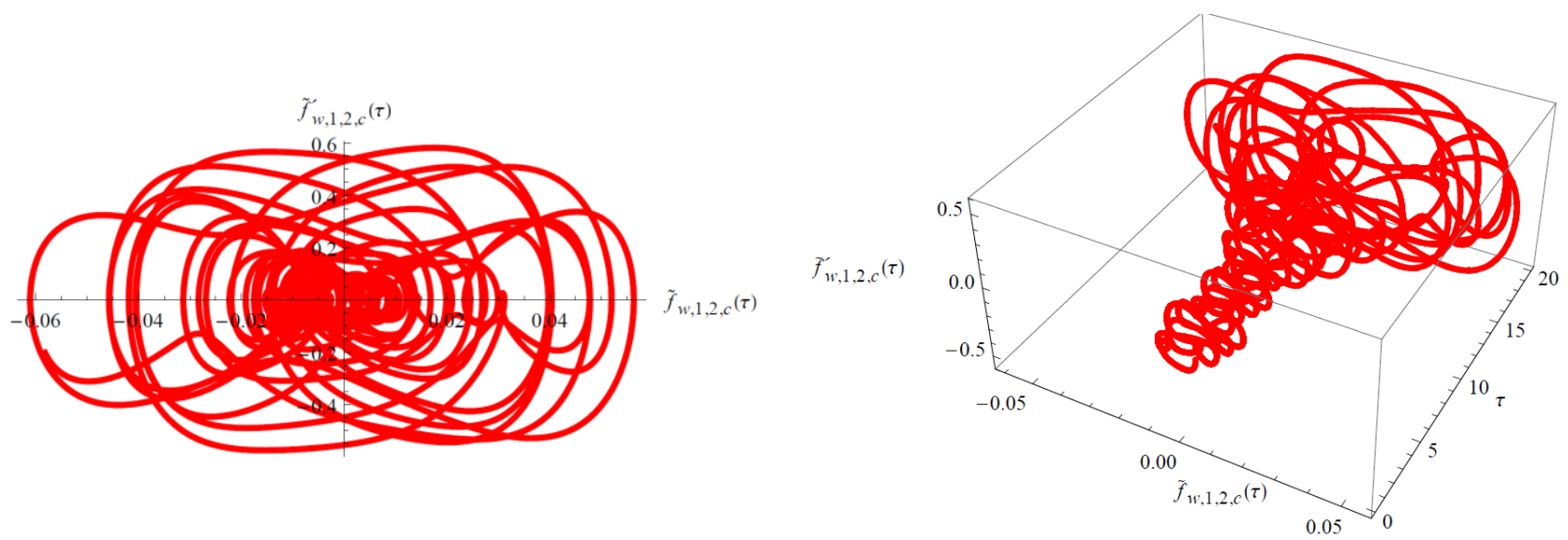

(c)
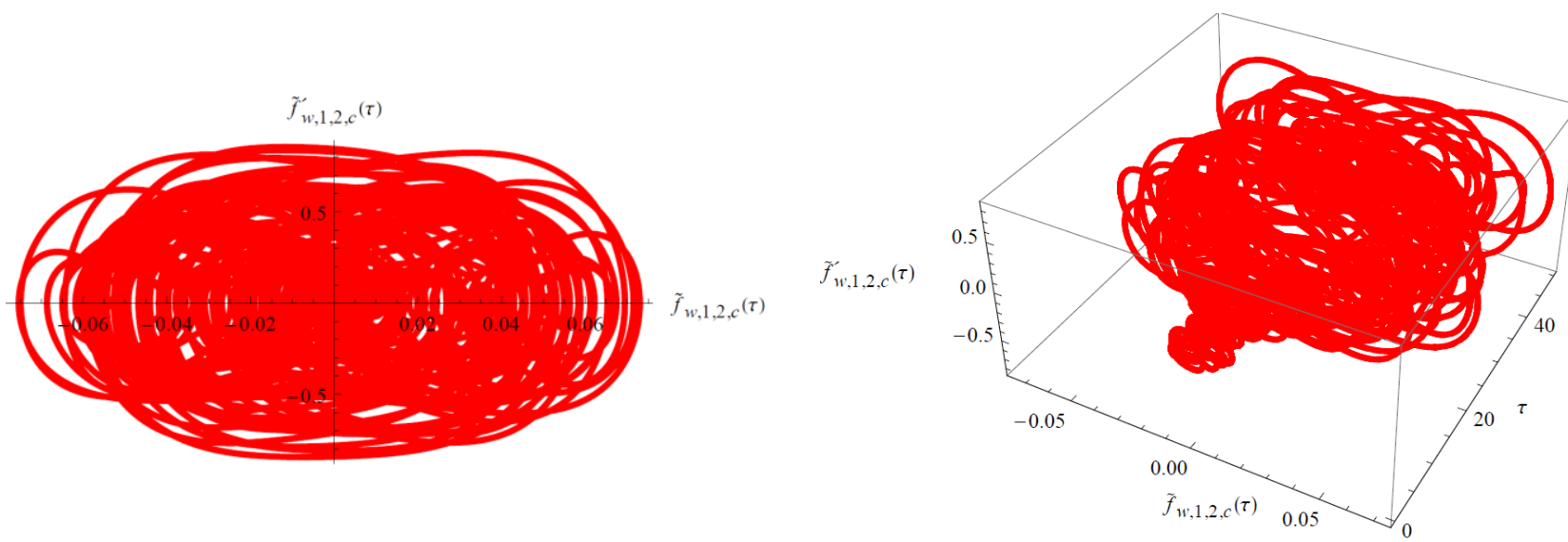

Figure 17. 2D and 3D phase portraits of the conjugate mode $(1,2, c)$. Free-free SWCNT of Table 1 . Nonlinear modal expansion (37). Modal initial conditions (38). (a) Time $\tau=5$. (b) Time $\tau=20$. (c) Time $\tau=50$. 


\subsubsection{Total energy distribution}

The evolution in time of the total energy distribution $\tilde{E}(\eta, \tau)$ of the conjugate modes $(1,2),(1,2, c)$ along the SWCNT axis, considering the circumferential coordinate $\theta=0$, is shown in Figure 18. An initial energy is imposed on the edge sections of the SWCNT $(\eta=(0,1))$ : differently from the linear case (Figure 9), this energy is not preserved in amplitude throughout the time.

(a)

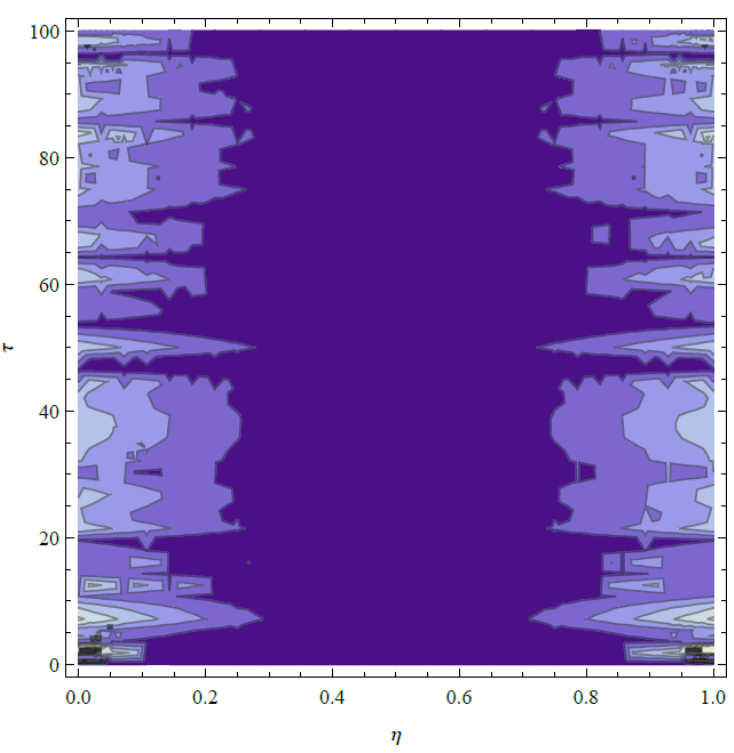

(b)

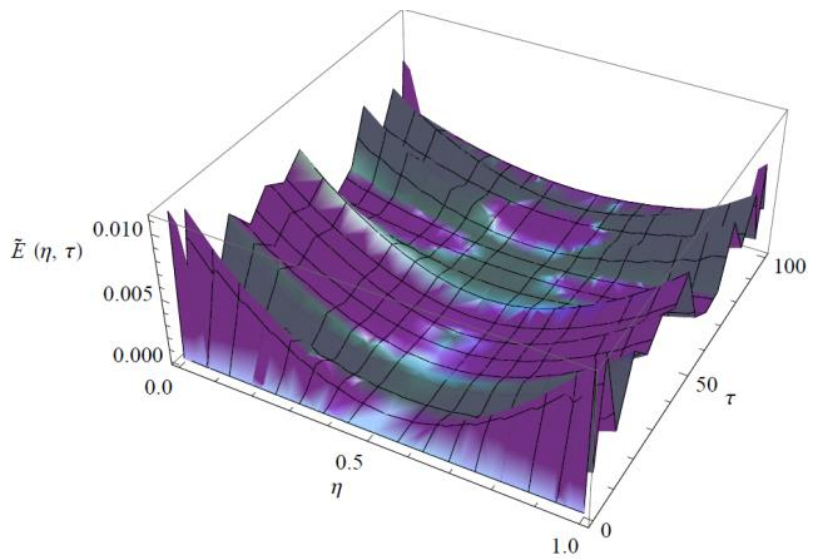

Figure 18. Contour plot (a) and 3D plot (b) of the total energy distribution $\tilde{E}(\eta, \tau)$ of the conjugate CFMs $(1,2),(1,2, c)$ along the SWCNT axis in time. Circumferential coordinate $\theta=0$. Free-free SWCNT of Table 1.

\subsubsection{Travelling wave response}

In the present section, the energy exchange between the conjugate CFMs $(1,2),(1,2, c)$ is studied. By considering the time histories of the two modes in the time range $\tau=(80,90)$, see Figure 19(a), it can be observed the presence of the phase difference $\Delta \phi=\pi$ between the two nonlinear responses in the specific time range $\tau=(84.27,85.17)$, see the enlargement of Figure 19(b).

The evolution of the total energy distribution $\tilde{E}(\eta, \theta, \tau)$ of the two conjugate CFMs $(1,2),(1,2, c)$ over the CNT surface $(\eta, \theta)$ in the time range $\tau=(84.27,85.17)$ is reported in Figures 20-25: differently from the linear case (Figures 10-11), the total energy distribution over the SWCNT surface evolves in the time in a complex pattern, where the total energy symmetry along the longitudinal direction and periodicity along the circumferential direction are still preserved. 
(a)

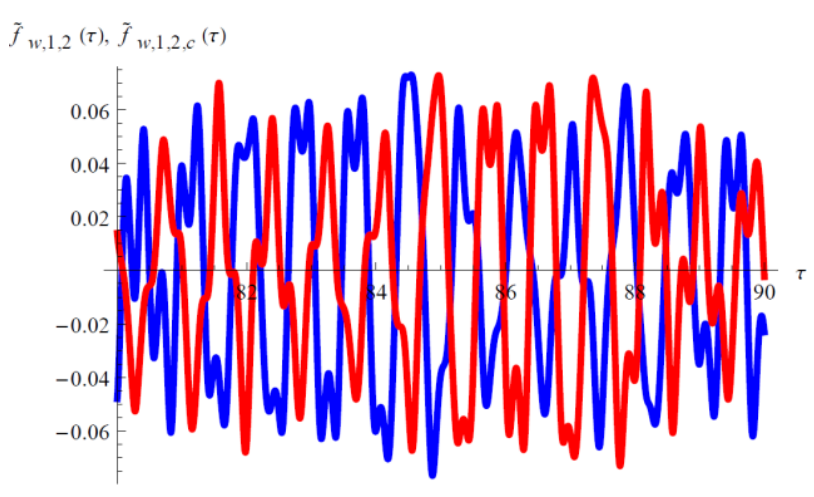

(b)

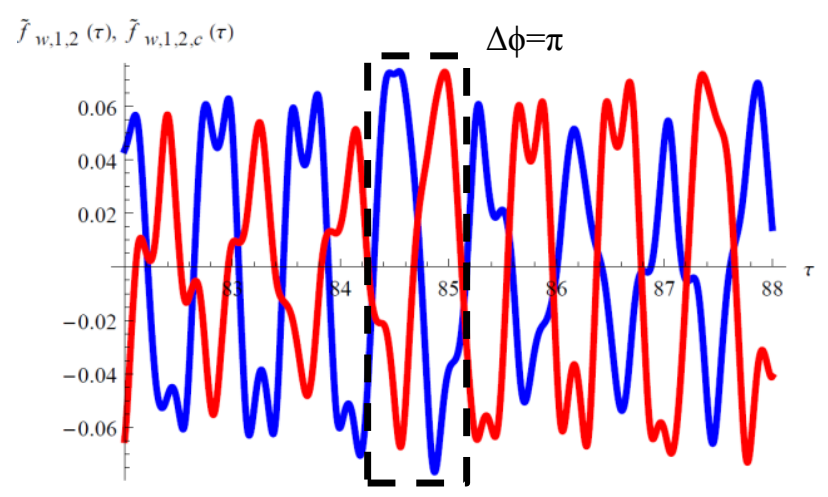

Figure 19. Time histories of the two conjugate modes $(1,2),(1,2, c)$. - Mode $(1,2)$. - Mode $(1,2, c)$.

(a) Time range $\tau=(80,90)$. (b) Time range $\tau=(84.27,85.17)$.

From Figures 20-25 it can be observed that the nonlinear resonance interaction between the two conjugate modes, in presence of the phase difference $\Delta \phi=\pi$, gives rise to an energy transfer between the two modes which generates a pure travelling wave moving circumferentially around the CNT. In Figures 20,23 the forward travelling wave from $\theta=0$ to $\theta=2 \pi$ in the time range $\tau=(84.27,84.57)$, corresponding to the initial ascending part of the nonlinear time history of the mode $(1,2)$ of Figure 19(b), is presented. In Figures 21,24 the backward travelling wave from $\theta=2 \pi$ to $\theta=0$ in the time range $\tau=(84.57,84.87)$, corresponding to the intermediate descending part of the nonlinear time history of the mode $(1,2)$ of Figure 19(b), is described. In Figures 22,25 the forward travelling wave from $\theta=0$ to $\theta=2 \pi$ in the time range $\tau=(84.87,85.17)$, corresponding to the final ascending part of the nonlinear time history of the mode $(1,2)$ of Figure 19(b), is shown.

It is reported in literature for the circular cylindrical shells that the presence of one couple of modes having the same mode shape but different angular orientation (such as the conjugate modes $(1,2)$, $(1,2, c)$ of the present paper), the first one described by $\cos n \theta$ (see radial displacement (17), directly excited mode), the second one described by $\sin n \theta$ (see radial displacement (21), slightly perturbed mode), in case of nonlinear resonance interaction between the modes, can lead to the appearance of a pure travelling wave moving along the circumferential direction of the shell. This phenomenon, which was observed also experimentally, is due to the axial symmetry of the structure and it occurs only in nonlinear field (that is a relevant difference vs. linear vibrations), see Refs. [59-60] for more details. To the Authors' best knowledge, the travelling wave phenomenon was not still observed for the SWCNTs, which are modelled in the present paper as continuous circular cylindrical shells in the framework of a thin shell theory. 
$\tau=84.27$

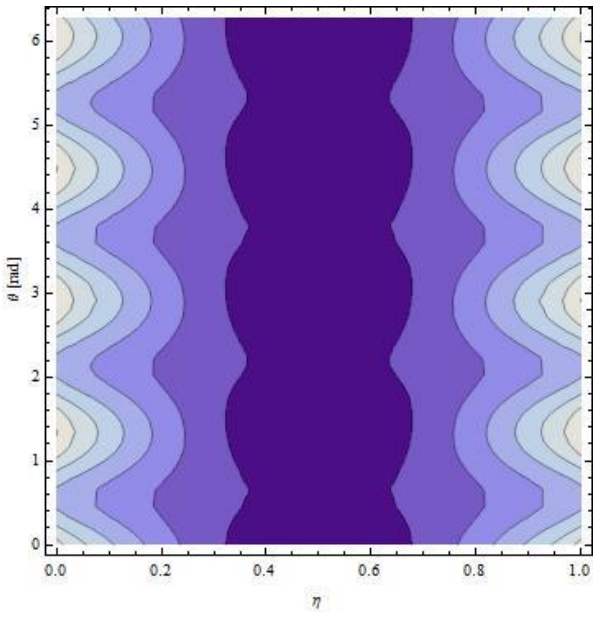

$\tau=84.39$

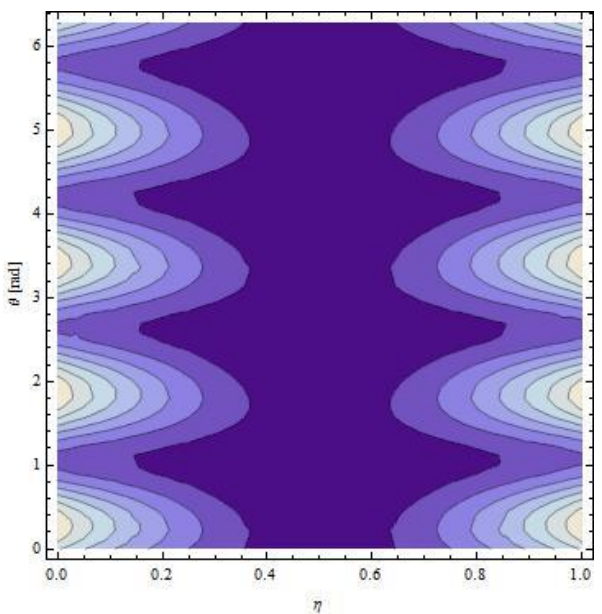

$\tau=84.51$

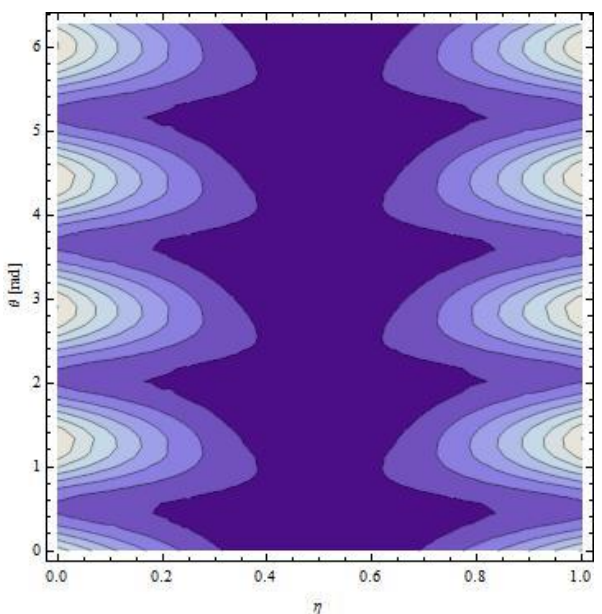

$\tau=84.33$

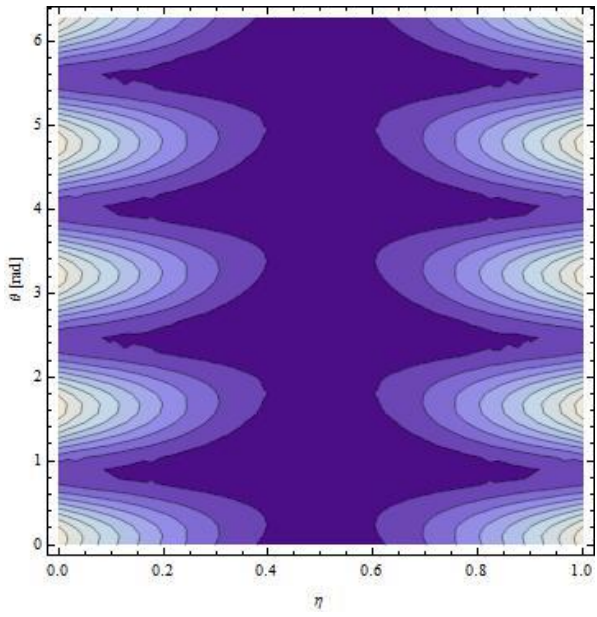

$\tau=84.45$

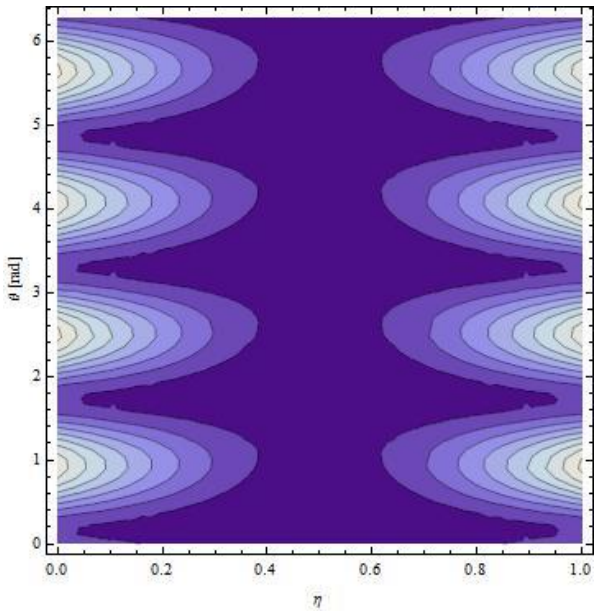

$\tau=84.57$

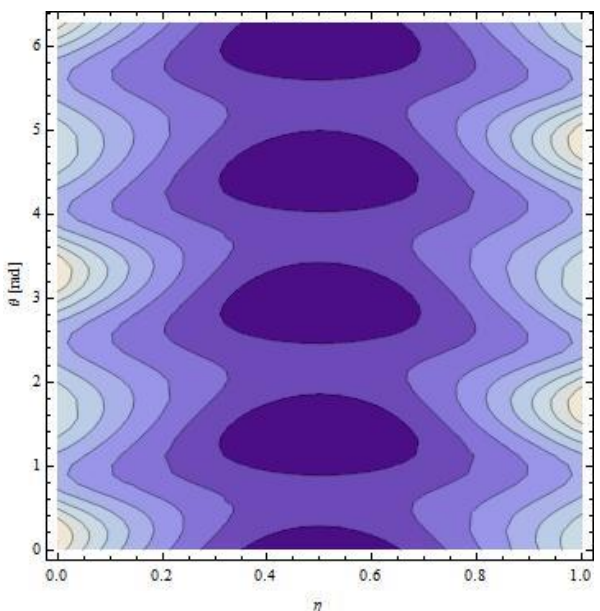

Figure 20. Contour plot of the total energy distribution $\tilde{E}(\eta, \theta, \tau)$ of the conjugate modes $(1,2),(1,2, c)$ over the SWCNT surface $(\eta, \theta)$. Forward travelling wave from $\theta=0$ to $\theta=2 \pi$ in the time range $\tau=(84.27,84.57)$. 
$\tau=84.57$

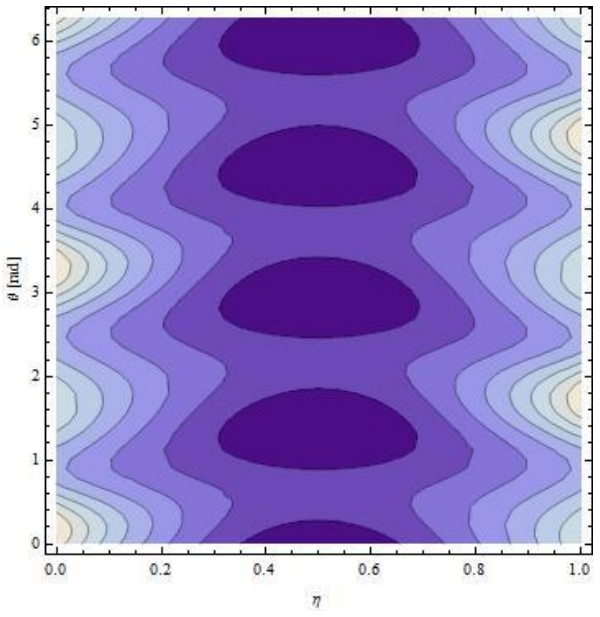

$\tau=84.69$

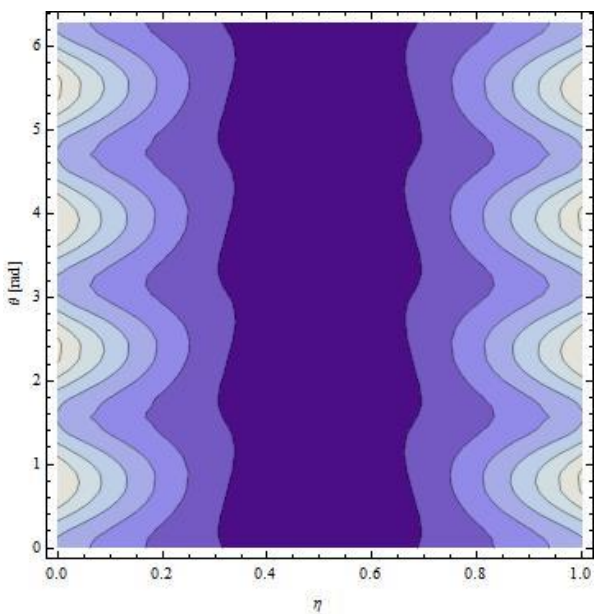

$\tau=84.81$

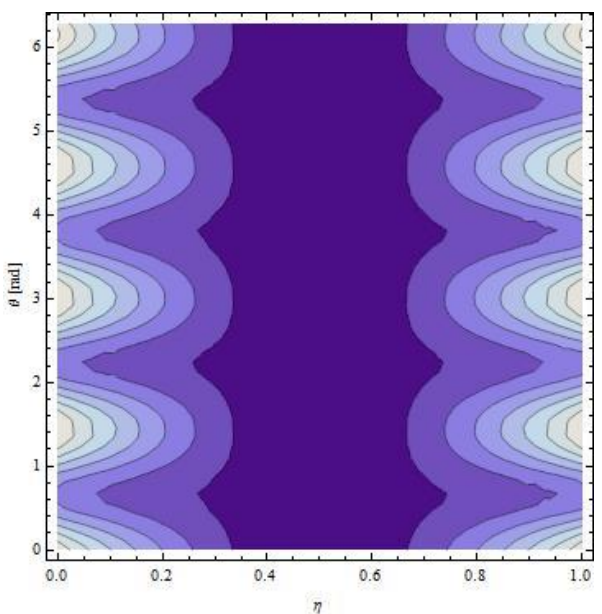

$\tau=84.63$

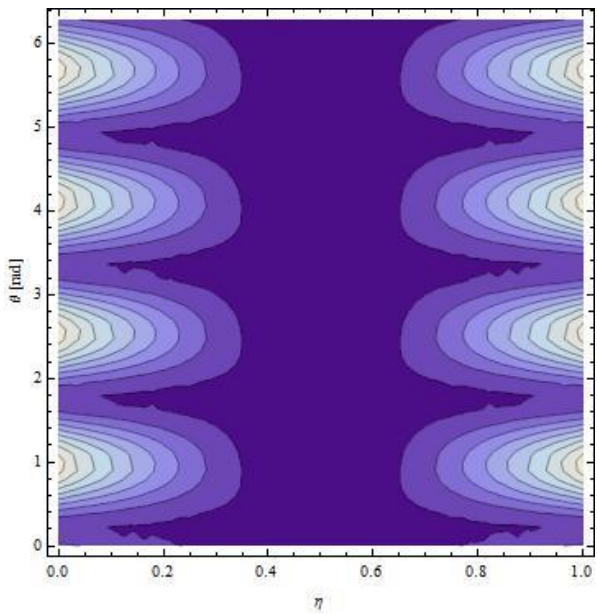

$\tau=84.75$

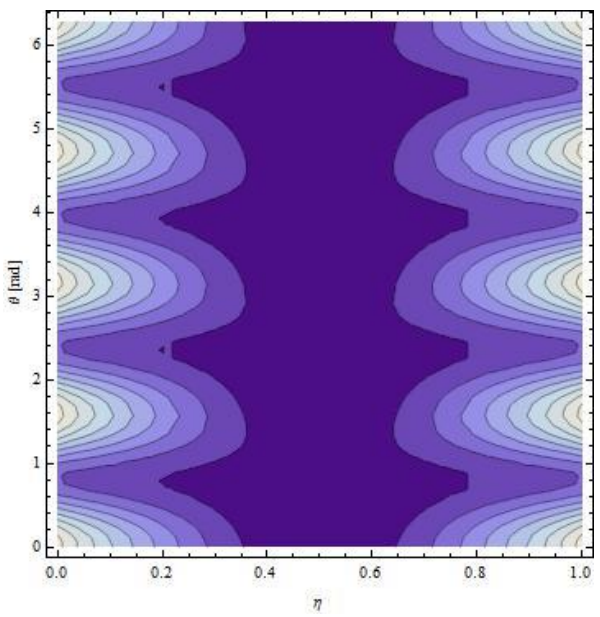

$\tau=84.87$

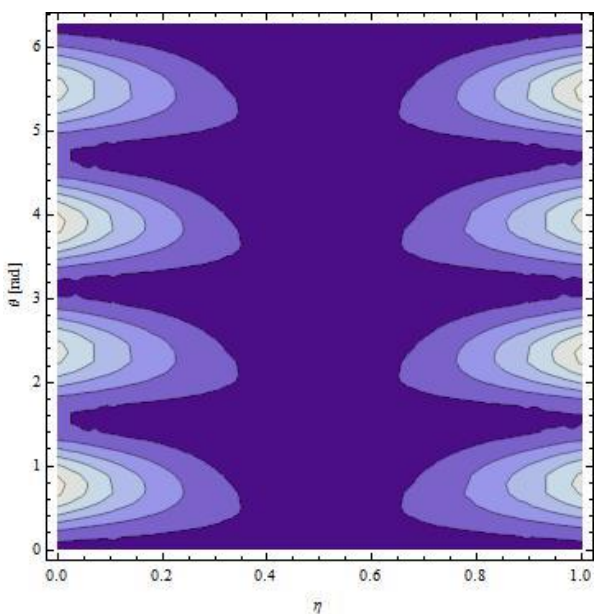

Figure 21. Contour plot of the total energy distribution $\tilde{E}(\eta, \theta, \tau)$ of the conjugate modes $(1,2),(1,2, c)$ over the SWCNT surface $(\eta, \theta)$. Backward travelling wave from $\theta=2 \pi$ to $\theta=0$ in the time range $\tau=(84.57,84.87)$. 
$\tau=84.87$

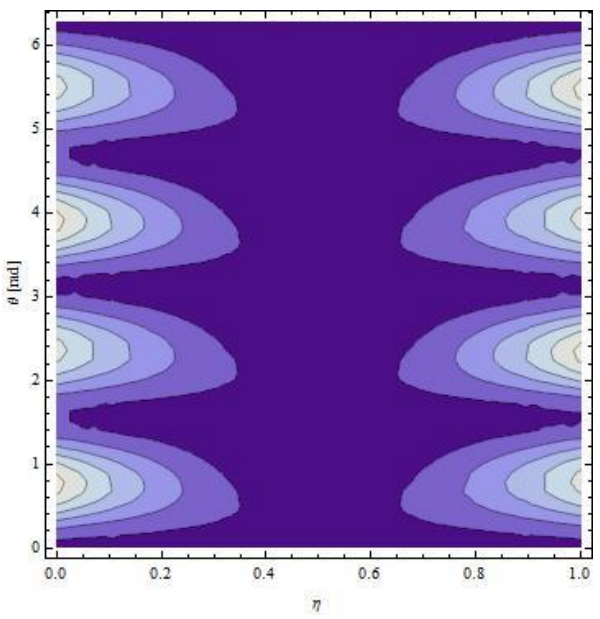

$\tau=84.99$

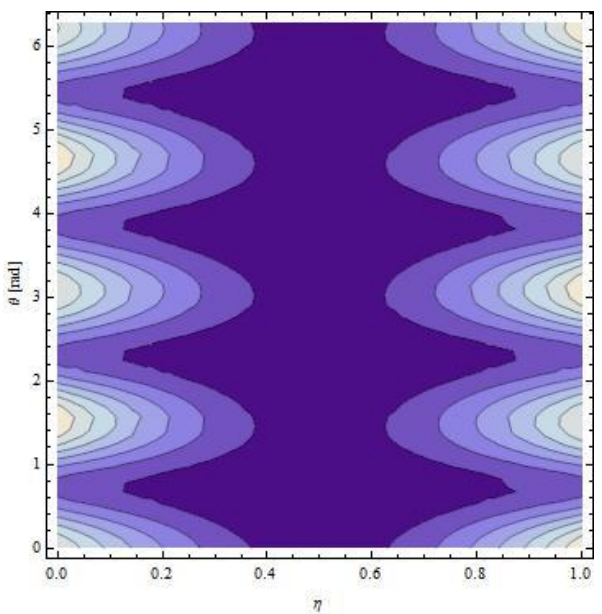

$\tau=85.11$

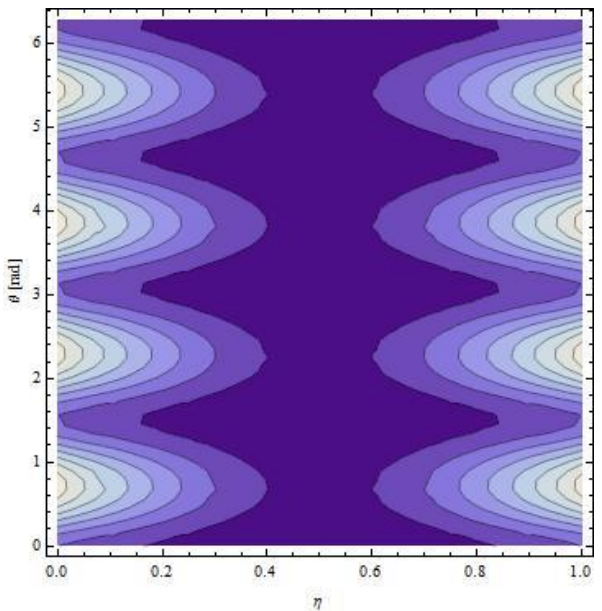

$\tau=84.93$

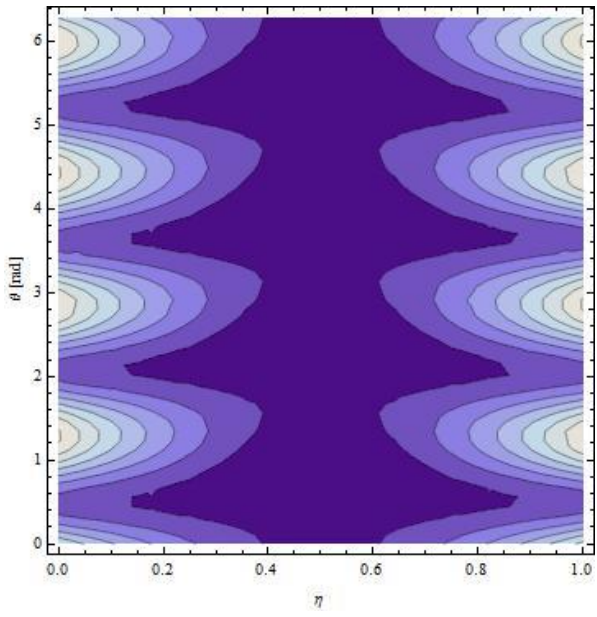

$\tau=85.05$

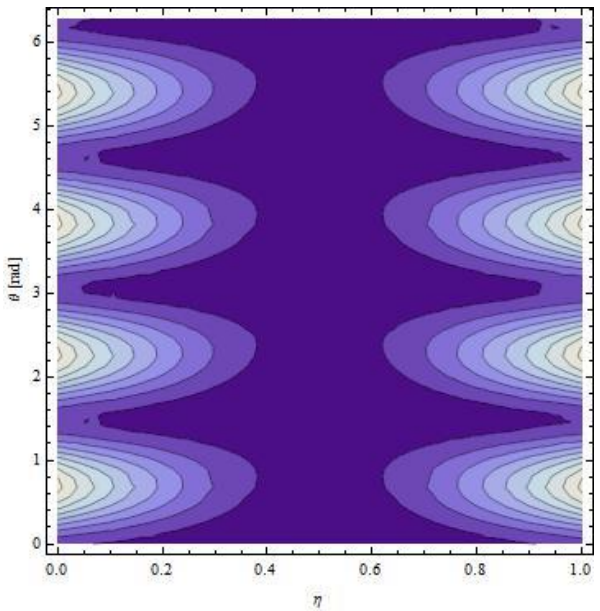

$\tau=85.17$

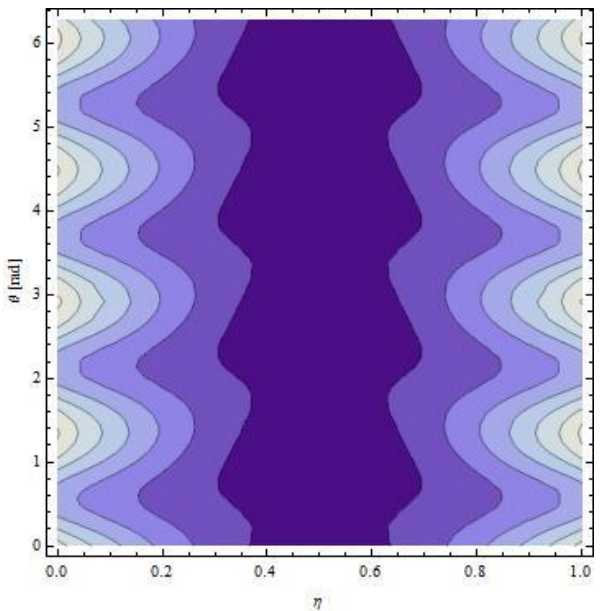

Figure 22. Contour plot of the total energy distribution $\tilde{E}(\eta, \theta, \tau)$ of the conjugate modes $(1,2),(1,2, c)$ over the SWCNT surface $(\eta, \theta)$. Forward travelling wave from $\theta=0$ to $\theta=2 \pi$ in the time range $\tau=(84.87,85.17)$. 
Nonlinear resonance interaction between conjugate circumferential flexural modes in single-walled carbon nanotubes

$\tau=84.27$

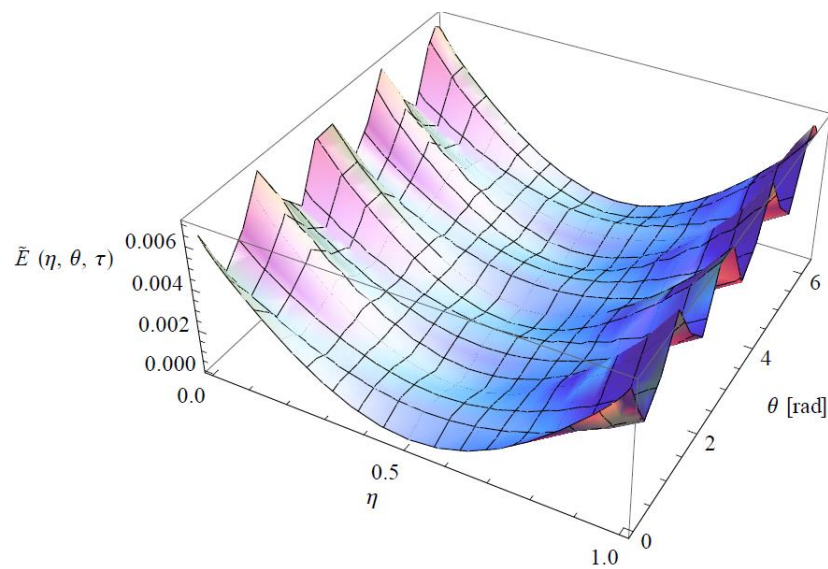

$\tau=84.39$

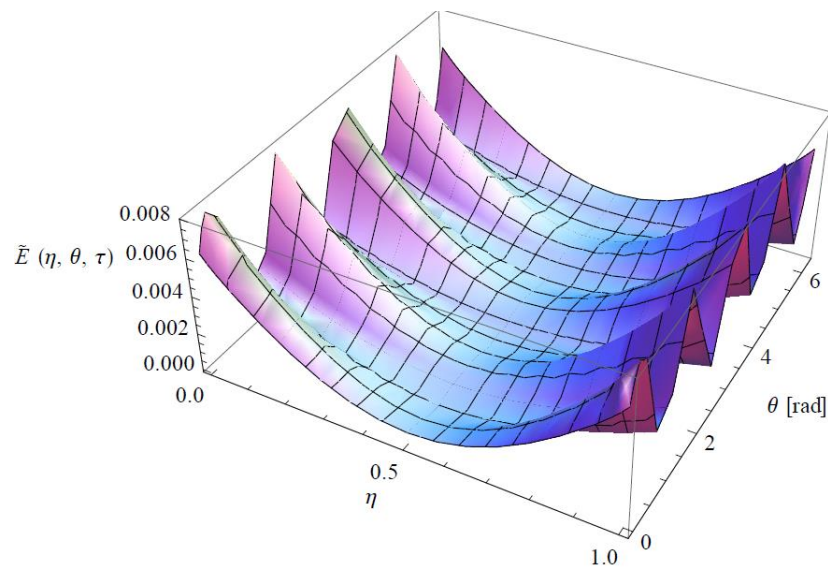

$\tau=84.51$

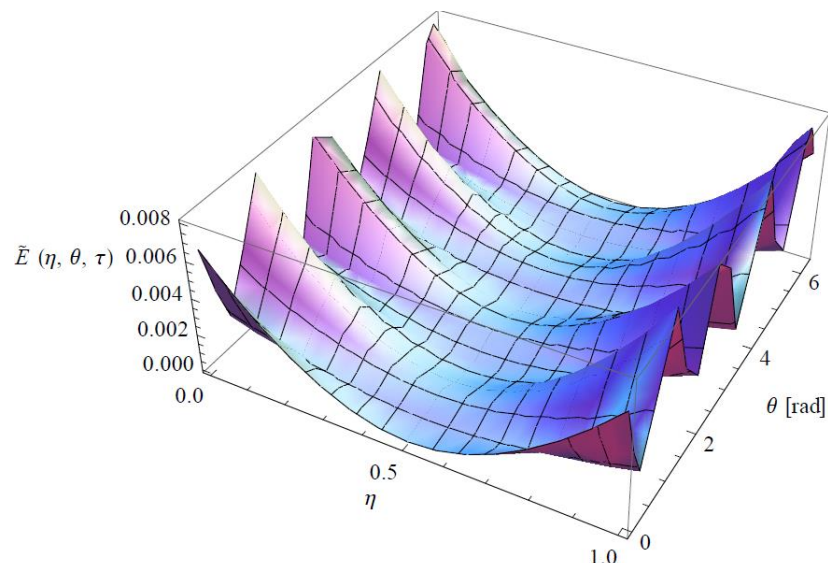

$\tau=84.33$

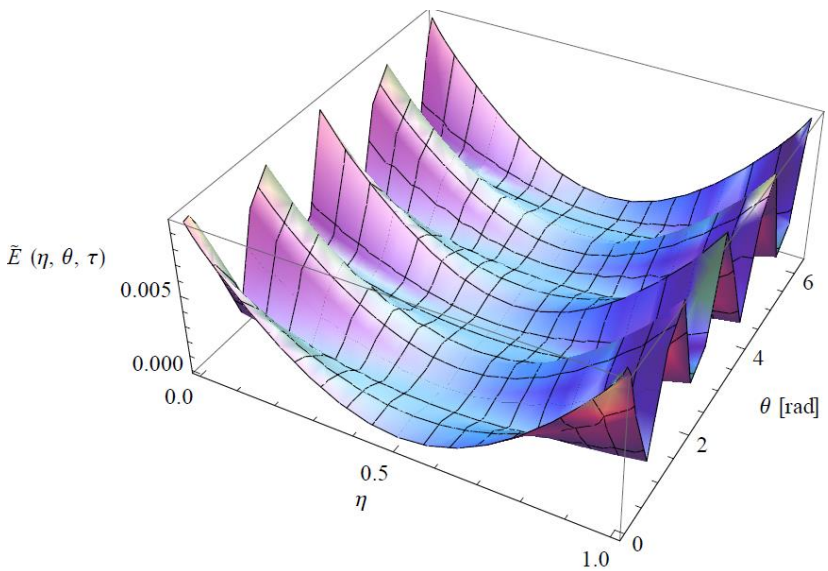

$\tau=84.45$

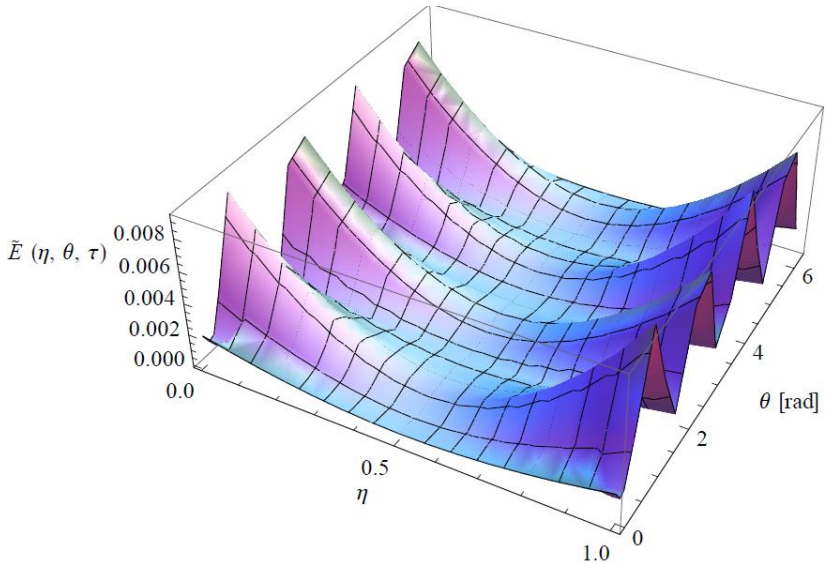

$\tau=84.57$

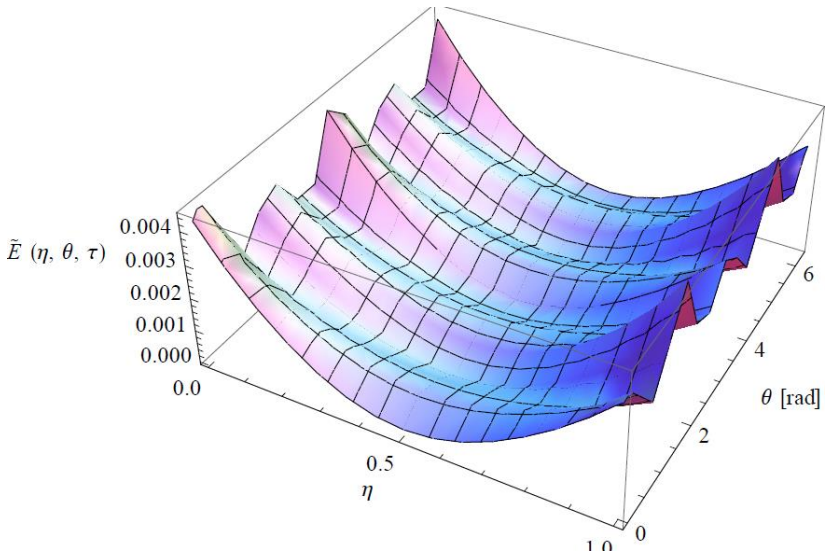

Figure 23. 3D plot of the total energy distribution $\tilde{E}(\eta, \theta, \tau)$ of the conjugate modes $(1,2),(1,2, c)$ over the SWCNT surface $(\eta, \theta)$. Forward travelling wave from $\theta=0$ to $\theta=2 \pi$ in the time range $\tau=(84.27,84.57)$. 
Nonlinear resonance interaction between conjugate circumferential flexural modes in single-walled carbon nanotubes

$\tau=84.57$

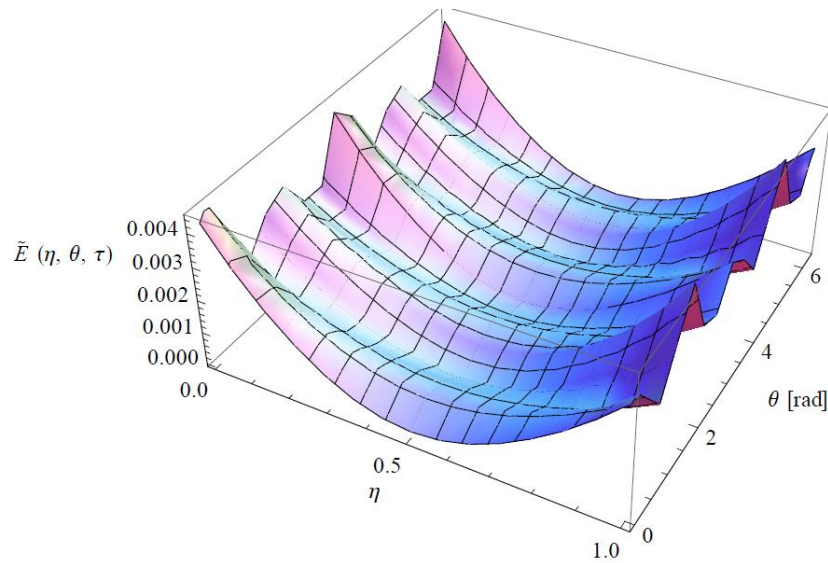

$\tau=84.69$

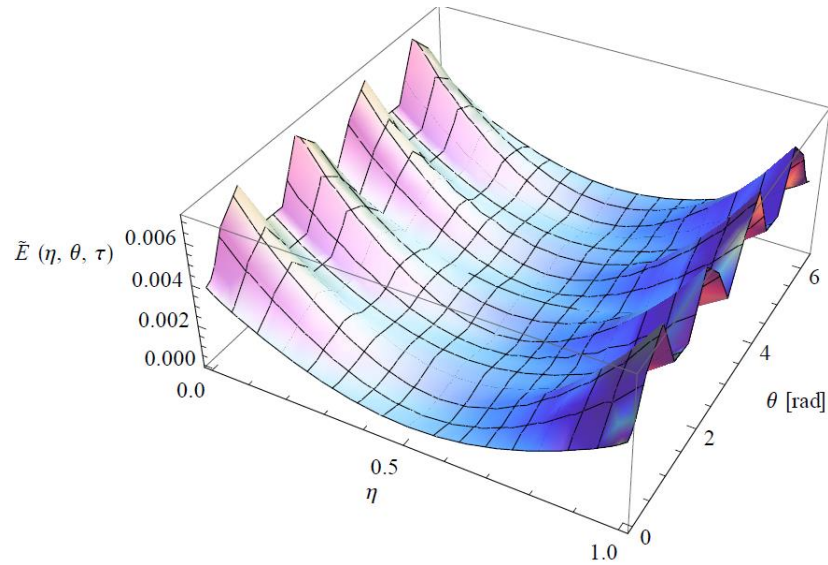

$\tau=84.81$

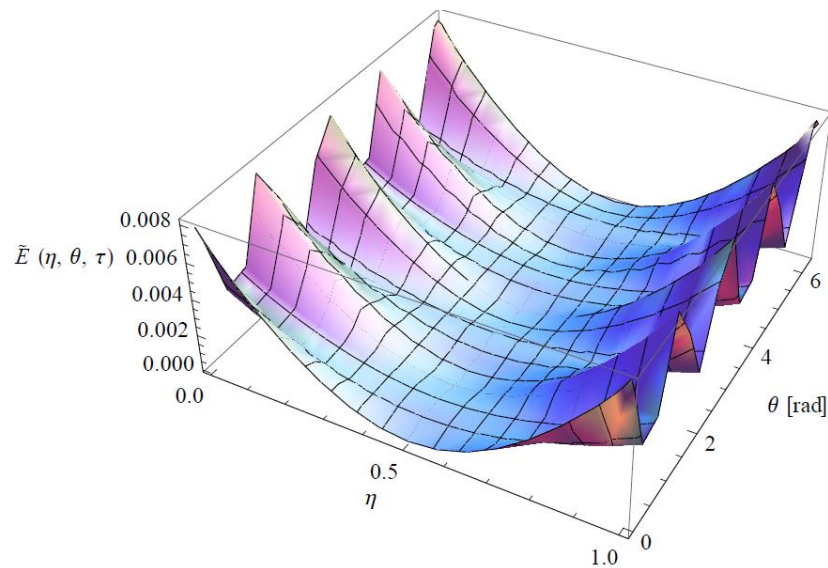

$\tau=84.63$

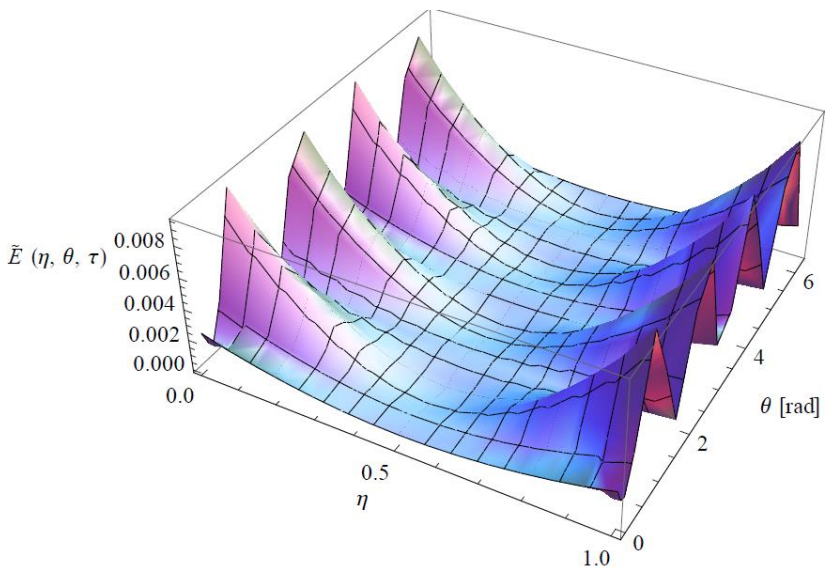

$\tau=84.75$

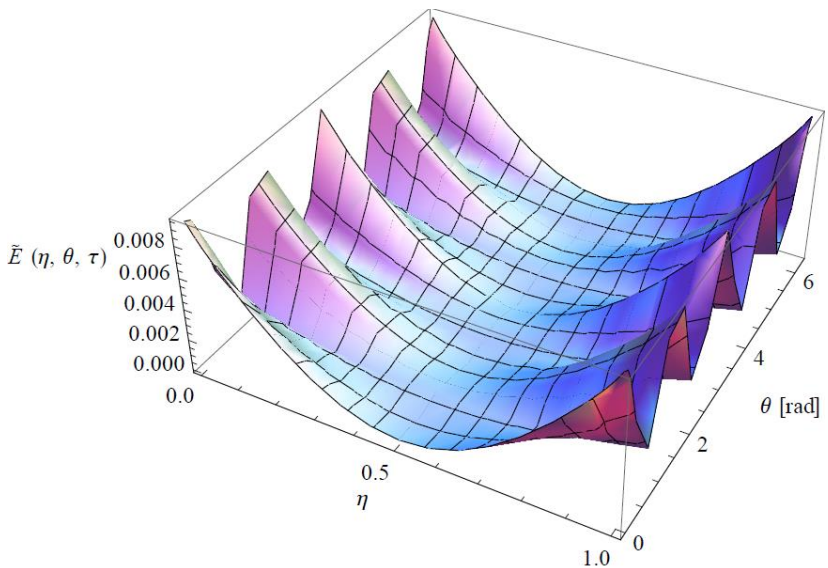

$\tau=84.87$

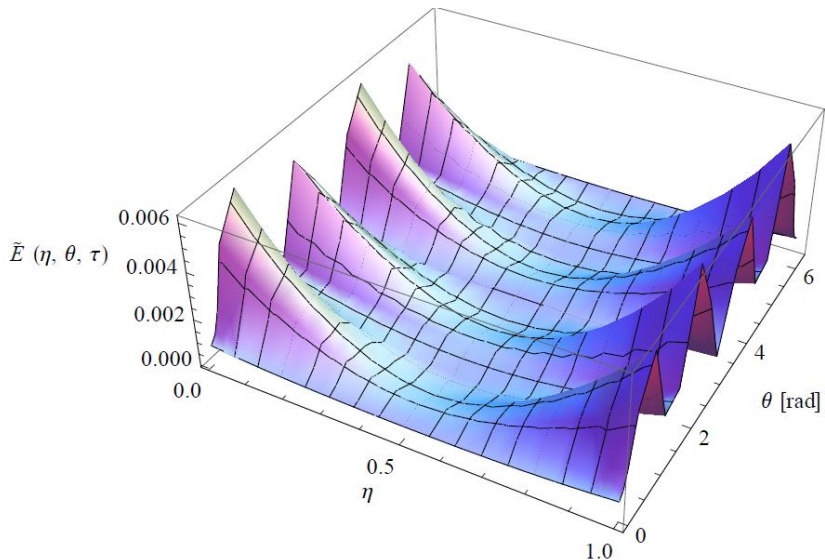

Figure 24. 3D plot of the total energy distribution $\tilde{E}(\eta, \theta, \tau)$ of the conjugate modes $(1,2),(1,2, c)$ over the SWCNT surface $(\eta, \theta)$. Backward travelling wave from $\theta=2 \pi$ to $\theta=0$ in the time range $\tau=(84.57,84.87)$. 
Nonlinear resonance interaction between conjugate circumferential flexural modes in single-walled carbon nanotubes

$\tau=84.87$

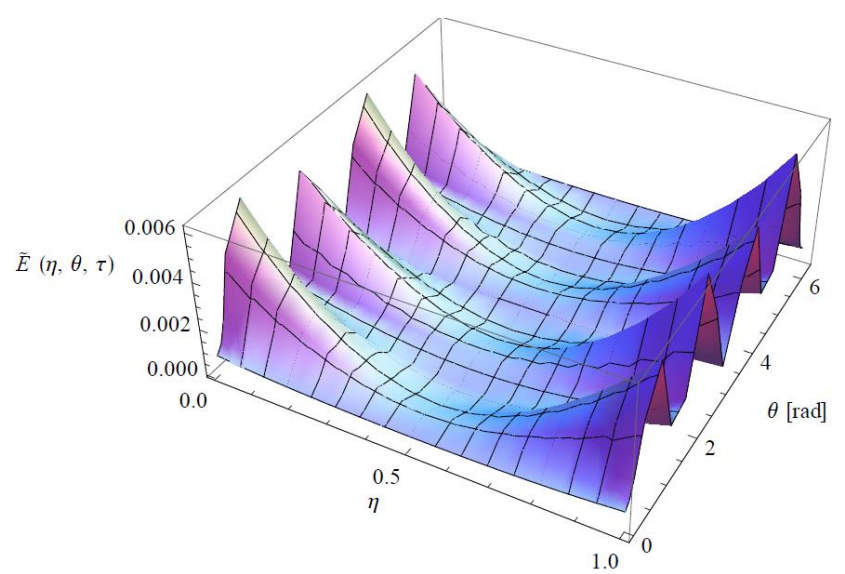

$\tau=84.99$

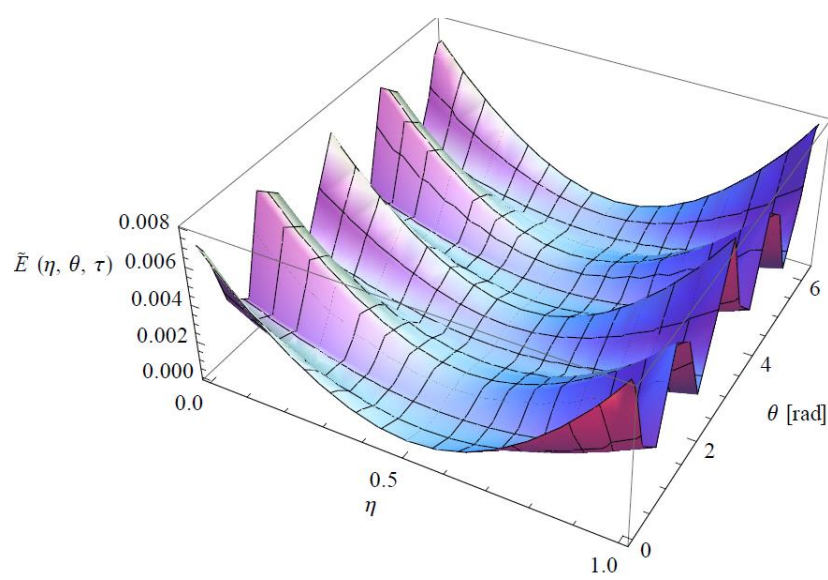

$\tau=85.11$

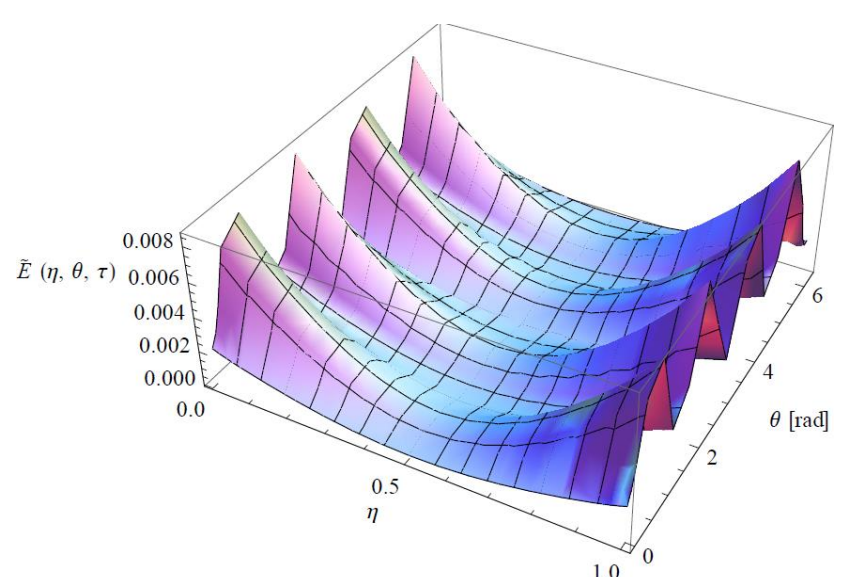

$\tau=84.93$

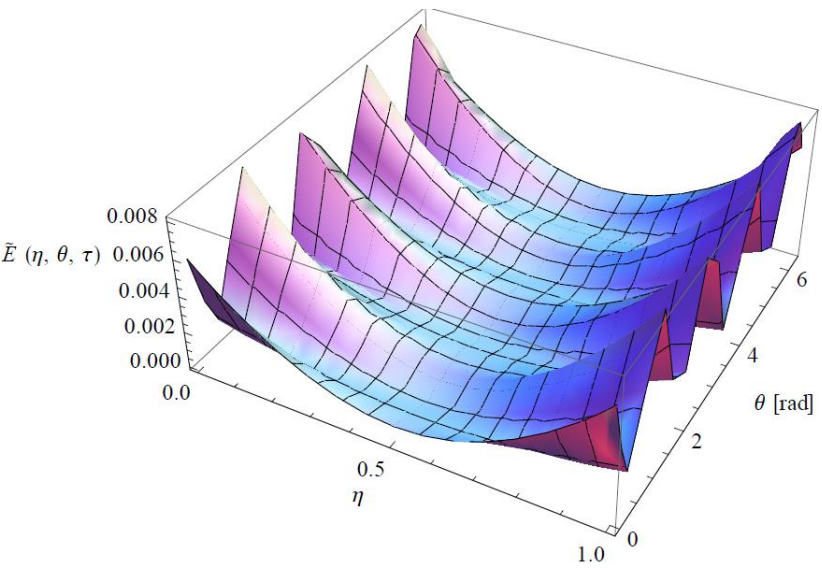

$\tau=85.05$

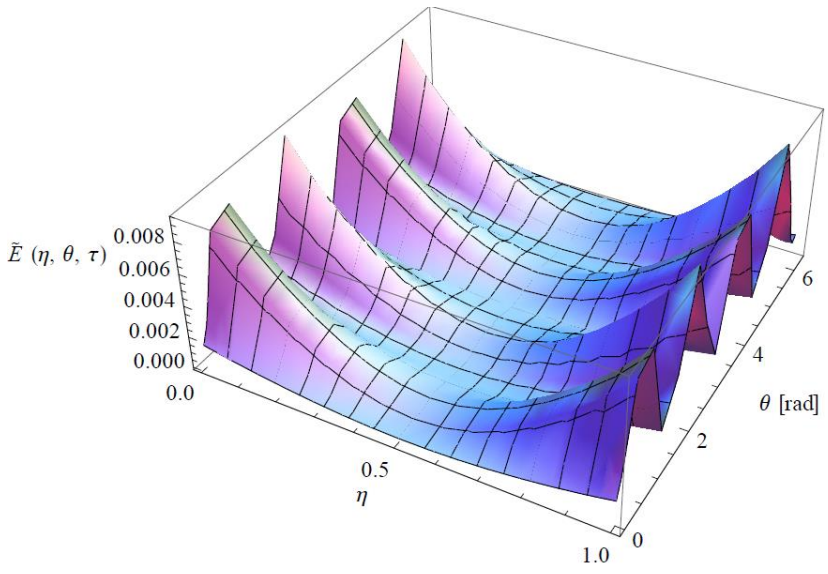

$\tau=85.17$

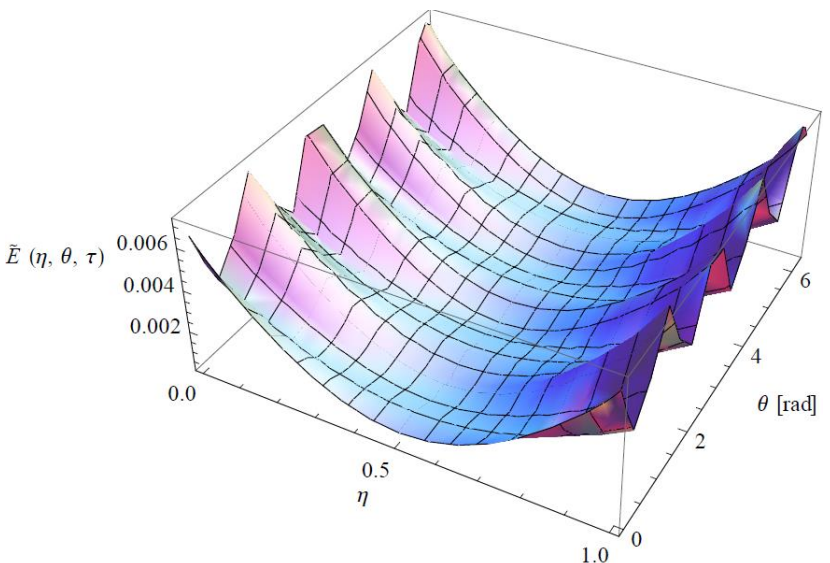

Figure 25. 3D plot of the total energy distribution $\tilde{E}(\eta, \theta, \tau)$ of the conjugate modes $(1,2),(1,2, c)$ over the SWCNT surface $(\eta, \theta)$. Forward travelling wave from $\theta=0$ to $\theta=2 \pi$ in the time range $\tau=(84.87,85.17)$. 


\section{Concluding remarks}

In the present paper the nonlinear resonance interaction between two conjugate CFMs of a SWCNT with free-free boundary conditions is analysed. The Sanders-Koiter shell theory is used to model the dynamics of the shell. The Rayleigh-Ritz method is applied to obtain approximated eigenfunctions. The Lagrange equations are used to get the system of nonlinear ordinary differential equations of motion, which is numerically solved by means of the implicit Runge-Kutta iterative method.

\section{1) Numerical results from the linear analysis}

The model proposed in this paper is validated in linear field by comparing the natural frequencies obtained with data retrieved from the pertinent literature: from these comparisons it can be observed that the present model is in very good agreement with the results of experimental analyses (RRS) and structural simulations (MDS).

The time histories, frequency spectra and phase portraits obtained demonstrate a periodic vibration of the SWCNT in linear field.

The total energy distribution over the carbon nanotube surface is periodic along the circumferential direction, symmetric along the longitudinal direction, where the initial energy imposed on the edge sections of the SWCNT is preserved in amplitude throughout the time; the total energy conservation of the considered undamped system is verified.

2) Numerical results from the nonlinear analysis

A convergence analysis is carried out to select the vibration modes to be added to the two directly excited CFMs providing an accurate description of the actual nonlinear behaviour of the SWCNT. The modal expansion obtained involves, in addition to the conjugate modes $(1,2)$ and $(1,2, c)$, also the conjugate asymmetric modes $(1,4),(1,4, c)$ and the axisymmetric mode $(1,0)(14$ dof model): it is confirmed the fundamental role of the additional asymmetric and axisymmetric modes to properly predict the nonlinear dynamics of the system.

The modal initial conditions activating the nonlinear response of the mode $(1,2, c)$ are investigated: this activation gives rise to an energy exchange between the two conjugate CFMs.

The time histories, frequency spectra and phase portraits obtained demonstrate a chaotic vibration of the SWCNT in nonlinear field.

The total energy distribution over the nanotube surface evolves in a complex pattern, maintaining symmetry along the longitudinal direction and periodicity along the circumferential direction, where the initial energy on the edge sections of the CNT is no longer preserved in amplitude in the time. The energy exchange between the two conjugate CFMs generates a pure travelling wave moving circumferentially around the SWCNT. 


\section{References}

[1] S. Iijima. Helical microtubules of graphitic carbon. Nature 354 (1991) 56-58.

[2] M.S. Dresselhaus, G. Dresselhaus, J.C. Charlier, E. Hernandez. Electronic, thermal and mechanical properties of carbon nanotubes. Philosophical Transactions of the Royal Society of London A 362 (2004) 2065-2098.

[3] Y. Huang, J. Wu, K.C. Hwang. Thickness of graphene and single-wall carbon nanotubes. Physical Review B 74 (2006) 245413 (9).

[4] N. Yao, V. Lordi. Young's modulus of single-walled carbon nanotubes. Journal of Applied Physics 84 (1998) 1939 (5).

[5] B. Mahar, C. Laslau, R. Yip, Y. Sun. Development of carbon nanotube-based sensors. A review. IEEE Sensors Journal 7 (2007) 266-284.

[6] H.M. Ouakad, M.I. Younis. Nonlinear Dynamics of Electrically Actuated Carbon Nanotube Resonators. Journal of Computational and Nonlinear Dynamics 5 (2010) 011009 (13).

[7] C. Li, E.T. Thostenson, T.W. Chou. Sensors and actuators based on carbon nanotubes and their composites: A review. Composites Science and Technology 68 (2008) 1227-1249.

[8] C. Li, T.W. Chou. Single-walled carbon nanotubes as ultrahigh frequency nano-mechanical resonators. Physical Review B 68 (2003) 073405 (3).

[9] R.R. Hartmann, J. Kono, M.E. Portnoi. Terahertz science and technology of carbon nanomaterials. Nanotechnology 25 (2014) 322001 (16).

[10] H.B. Peng, C.W. Chang, S. Aloni, T.D. Yuzvinsky, A. Zettl. Ultrahigh Frequency Nanotube Resonators. Physical Review Letters 97 (2006) 087203 (4).

[11] A. Jorio, R. Saito, J.H. Hafner, C.M. Lieber, M. Hunter, T. McClure, G. Dresselhaus, M.S. Dresselhaus. Structural $(n, m)$ determination of isolated single-wall carbon nanotubes by resonant Raman scattering. Physical Review Letters 86 (2001) 1118-1121.

[12] A. Jorio, R. Saito, G. Dresselhaus, M.S. Dresselhaus. Determination of nanotubes properties by Raman spectroscopy. Philosophical Transactions of the Royal Society of London A 362 (2004) 2311-2336.

[13] A. Rao, E. Richter, S. Bandow, B. Chase, P. Eklund, K. Williams, S. Fang, K. Subbaswamy, M. Menon, A. Thess, R.E. Smalley, G. Dresselhaus, M.S. Dresselhaus. Diameter-Selective Raman Scattering from Vibrational Modes in Carbon Nanotubes. Science 275 (197) 187- 191.

[14] S. Bandow, S. Asaka, Y. Saito, A.M. Rao, L. Grigorian, E. Richter, P.C. Eklund. Effect of the Growth Temperature on the Diameter Distribution and Chirality of Single-Wall Carbon Nanotubes. Physical Review Letters 80 (1998) 3779-3782. 
[15] M.S. Dresselhaus, G. Dresselhaus, A. Jorio, A.G. Souza Filho, R. Saito. Raman spectroscopy on isolated single wall carbon nanotubes. Carbon 40 (2002) 2043-2061.

[16] Y.Y. Zhang, C.M. Wang, V.B. Tan. Assessment of Timoshenko Beam Models for Vibrational Behaviour of Single-Walled Carbon Nanotubes using Molecular Dynamics. Advances in Applied Mathematics and Mechanics 1 (2009) 89-106.

[17] W.H. Duan, C.M. Wang, Y.Y. Zhang. Calibration of nonlocal scaling effect parameter for free vibration of carbon nanotubes by molecular dynamics simulations. Journal of Applied Physics 101 (2007) 024305 (7).

[18] R. Ansari, S. Ajori, B. Arash. Vibrations of single and double-walled carbon nanotubes with layerwise boundary conditions: A molecular dynamics study. Current Applied Physics 12 (2012) 707-711.

[19] S.S. Gupta, F.G. Bosco, R.C. Batra. Wall thickness and elastic moduli of single-walled carbon nanotubes from frequencies of axial, torsional and inextensional modes of vibration. Computational Materials Science 47 (2010) 1049-1059.

[20] N. Yao, Vincenzo Lordi. Young's modulus of single-walled carbon nanotubes. Journal of Applied Physics 84 (1998) 1939-1943.

[21] I. Elishakoff, D. Pentaras. Fundamental natural frequencies of double-walled carbon nanotubes. Journal of Sound and Vibration 322 (2009) 652-664.

[22] J. Yoon, C.Q. Ru, A. Mioduchowski. Terahertz vibration of short carbon nanotubes modelled as Timoshenko beams. Journal of Applied Mechanics 72 (2005) 10-17.

[23] C.M. Wang, V.B. Tan, Y.Y. Zhang. Timoshenko beam model for vibration analysis of multiwalled carbon nanotubes. Journal of Sound and Vibration 294 (2006)1060-1072.

[24] D. Pentaras, I. Elishakoff. Free vibration of triple-walled carbon nanotubes. Acta Mechanica 221 (2011) 239-249.

[25] I. Elishakoff, D. Pentaras. Natural frequencies of carbon nanotubes based on simplified BresseTimoshenko theory. Journal of Computational and Theoretical Nanoscience 6 (2009) 1527-1531.

[26] I. Elishakoff, D. Pentaras. Buckling of double-walled carbon nanotubes. Applied Science Letters 2 (2009) 372-376.

[27] I. Elishakoff, D. Pentaras, K. Dujat, M. Lemaire. Exact Solution for natural frequencies of clamped-clamped double walled carbon nanotubes. Philosophical Magazine Letters 91 (2011) 1-17. [28] I. Elishakoff, K. Dujat, M. Lemaire. Buckling of double-walled carbon nanotubes. Vietnam Journal of Mechanics 34 (2012) 217-224.

[29] I. Elishakoff, S. Bucas. Buckling of a clamped-free double-walled carbon nanotube by the Bubnov-Galerkin method. Journal of Applied Mechanics 80 (2013) 011004. 
[30] I. Elishakoff, C. Versaci, G. Muscolino. Effective stiffness and effective mass of the doublewalled carbon nanotube sensor. Journal of Nanotechnology in Engineering and Medicine 2 (2011) 011008.

[31] I. Elishakoff, C. Versaci, G. Muscolino. Clamped-free double-walled carbon nanotube-based mass sensor. Acta Mechanica 219 (2011) 29-43.

[32] I. Elishakoff, C. Versaci, N. Maugeri, G. Muscolino. Clamped-free single-walled carbon nanotube-based mass sensor treated as Bernoulli-Euler beam. Journal of Nanotechnology in Engineering and Medicine 2 (2011) 021001.

[33] D. Pentaras, I. Elishakoff. Dynamic deflection of a single-walled carbon nanotube under ballistic impact loading. Journal of Nanotechnology in Engineering and Medicine 2 (2012) 041002.

[34] K.M. Liew, Q. Wang. Analysis of wave propagation in carbon nanotubes via elastic shell theories. International Journal of Engineering Science 45 (2007) 227-241.

[35] C.Y. Wang, C.Q. Ru, A. Mioduchowski. Applicability and Limitations of Simplified Elastic Shell Equations for Carbon Nanotubes. Journal of Applied Mechanics 71 (2004) 622-631.

[36] E. Ghavanloo, S.A. Fazelzadeh. Vibration characteristics of single-walled carbon nanotubes based on anisotropic elastic shell model including chirality effect. Applied Mathematical Modelling 36 (2012) 4988-5000.

[37] N. Silvestre, C.M. Wang, Y.Y. Zhang, Y. Xiang. Sanders shell model for buckling of singlewalled carbon nanotubes with small aspect ratio. Composite Structures 93 (2011) 1683-1691.

[38] M. Strozzi, L.I. Manevitch, F. Pellicano, V.V. Smirnov, D.S. Shepelev. Low-frequency linear vibrations of single-walled carbon nanotubes: Analytical and numerical models. Journal of Sound and Vibration 333 (2014) 2936-2957.

[39] A.W. Leissa. Vibrations of Shells. Government Printing Office, Washington DC, 1973.

[40] N. Yamaki. Elastic Stability of Circular Cylindrical Shells. North-Holland, Amsterdam, 1984.

[41] B.I. Yakobson, C.J. Brabec, J. Bernholc. Nanomechanics of Carbon Tubes: Instabilities beyond Linear Response. Physical Review Letters 76 (1996) 2511-2514.

[42] S.A. Fazelzadeh, E. Ghavanloo. Nonlocal anisotropic elastic shell model for vibrations of single-walled carbon nanotubes with arbitrary chirality. Composite Structures 94 (2012) 1016-1022. [43] R. Ansari, A. Shahabodini, H. Rouhi. A thickness-independent nonlocal shell model for describing the stability behaviour of carbon nanotubes under compression. Composite Structures 100 (2013) 323-331.

[44] Y.G. Hu, K.M. Liew, Q. Wang, X.Q. He, B.I. Yakobson. Nonlocal shell model for elastic wave propagation in single and double-walled carbon nanotubes. Journal of the Mechanics and Physics of Solids 56 (2008) 3475-3485. 
[45] L. Wang, H. Hu, W. Guo. Validation of the non-local elastic shell model for studying longitudinal waves in single-walled carbon nanotubes. Nanotechnology 17 (2006) 1408-1415.

[46] F. Pellicano. Vibrations of circular cylindrical shells: theory and experiments. Journal of Sound and Vibration 303 (2007) 154-170.

[47] M. Amabili. Nonlinear Vibrations and Stability of Shells and Plates. Cambridge University Press. New York (2008).

[48] F. Pellicano. Dynamic instability of a circular shell carrying a top mass under base excitation: Experiments and theory. International Journal of Solids and Structures 48 (2011) 408-427.

[49] M. Amabili. Theory and experiments for large-amplitude vibrations of empty and fluid-filled circular cylindrical shells with imperfections. Journal of Sound and Vibration 262 (2003) 921-975.

[50] F. Pellicano, M. Barbieri, A. Zippo, M. Strozzi. Experiments on shells under base excitation. Journal of Sound and Vibration 369 (2016) 209-227.

[51] M. Strozzi, F. Pellicano. Nonlinear vibrations of functionally graded cylindrical shells. ThinWalled Structures 67 (2013) 63-77.

[52] F. Pellicano, M. Amabili, M.P. Paidoussis. Effect of the geometry on the non-linear vibration of circular cylindrical shells. International Journal of Nonlinear Mechanics 37 (2002) 1181-1198.

[53] G. Catellani, F. Pellicano, D. Dall'Asta, M. Amabili. Parametric instability of a circular cylindrical shell with geometric imperfections. Computers and Structures 82 (2004) 2635-2645.

[54] V.V. Smirnov, L.I. Manevitch. Semi-inverse method in nonlinear mechanics: application to couple shell- and beam-type oscillations of single-walled carbon nanotubes. Nonlinear Dynamics (2017) 1-14.

[55] M. Strozzi, V.V. Smirnov, L.I. Manevitch, M. Milani, F. Pellicano. Nonlinear vibrations and energy exchange of single-walled carbon nanotubes. Circumferential flexural modes. Journal of Sound and Vibration 381 (2016) 156-178.

[56] L.I. Manevitch, V.V. Smirnov, M. Strozzi, F. Pellicano. Nonlinear optical vibrations of singlewalled carbon nanotubes. International Journal of Non-Linear Mechanics 94 (2017) 351-361.

[57] M. Strozzi, V.V. Smirnov, L.I. Manevitch, F. Pellicano. Nonlinear vibrations and energy exchange of single-walled carbon nanotubes. Radial breathing modes. Composite Structures 184 (2018) 613-632.

[58] V.V. Smirnov, D.S. Shepelev, L.I. Manevitch. Localization of Low-Frequency Oscillations in Single-Walled Carbon Nanotubes. Physical Review Letters 113 (2014) 135502 (4).

[59] M. Amabili. A comparison of shell theories for large-amplitude vibrations of circular cylindrical shells: Lagrangian approach. Journal of Sound and Vibration 264 (2003) 1091-1125. 
[60] M. Amabili, P. Balasubramanian, G. Ferrari. Travelling wave and non-stationary response in nonlinear vibrations of water-filled circular cylindrical shells: Experiments and simulations. Journal of Sound and Vibration 381 (2016) 220-245. 